\title{
AVTA Federal Fleet PEV Readiness Data Logging and Characterization Study for the National Park Service: Grand Canyon National Park
}

Stephen Schey Jim Francfort Ian Nienhueser

August 2014

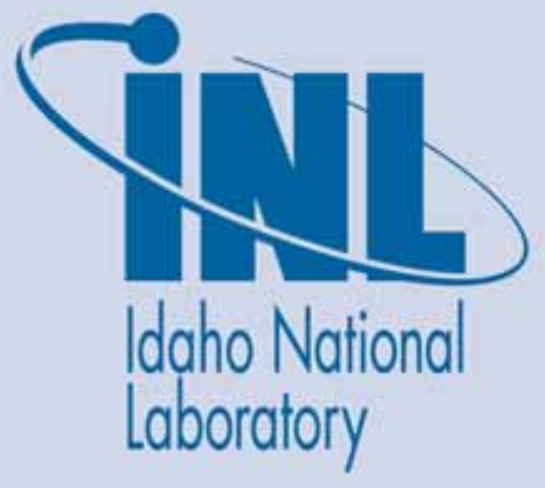

The INL is a U.S. Department of Energy National Laboratory operated by Battelle Energy Alliance 


\section{DISCLAIMER}

This information was prepared as an account of work sponsored by an agency of the U.S. Government. Neither the U.S. Government nor any agency thereof, nor any of their employees, makes any warranty, expressed or implied, or assumes any legal liability or responsibility for the accuracy, completeness, or usefulness, of any information, apparatus, product, or process disclosed, or represents that its use would not infringe privately owned rights. References herein to any specific commercial product, process, or service by trade name, trade mark, manufacturer, or otherwise, does not necessarily constitute or imply its endorsement, recommendation, or favoring by the U.S. Government or any agency thereof. The views and opinions of authors expressed herein do not necessarily state or reflect those of the U.S. Government or any agency thereof. 
INL/EXT-14-33267

\title{
AVTA Federal Fleet PEV Readiness Data Logging and Characterization Study for the National Park Service: Grand Canyon National Park
}

\author{
Stephen Schey \\ Jim Francfort ${ }^{2}$ \\ lan Nienhueser ${ }^{3}$
${ }^{1}$ Stephen Schey, Project Manager, Infrastructure Planning and Analysis, Intertek Testing Services, North America, Phoenix, AZ
${ }^{2}$ Jim Francfort, Vehicle Systems Principal Investigator, Idaho National Laboratory operated by Battelle energy Alliance, Idaho Falls, ID
${ }^{3}$ Ian Nienhueser, Data Analyst, Infrastructure Planning and Analysis, Intertek Testing Services, \\ North America, Phoenix, AZ
}

August 2014

\section{Idaho National Laboratory \\ Idaho Falls, Idaho 83415}

http://avt.inl.gov

Prepared for the

U.S. Department of Energy

Office of Nuclear Energy

Under DOE Idaho Operations Office

Contract DE-AC07-05ID14517 



\section{ABSTRACT}

Battelle Energy Alliance, LLC, managing and operating contractor for the U.S. Department of Energy's Idaho National Laboratory, is the lead laboratory for U.S. Department of Energy Advanced Vehicle Testing. Battelle Energy Alliance, LLC contracted with Intertek Testing Services, North America (Intertek) to collect and evaluate data on federal fleet operations as part of the Advanced Vehicle Testing Activity's Federal Fleet Vehicle Data Logging and Characterization study. The Advanced Vehicle Testing Activity study seeks to collect and evaluate data to validate the utilization of advanced plug-in electric vehicle (PEV) transportation.

This report focuses on the Grand Canyon National Park (GCNP) fleet to identify daily operational characteristics of select vehicles and report findings on vehicle and mission characterizations to support the successful introduction of PEVs into the agencies' fleets.

Individual observations of these selected vehicles provide the basis for recommendations related to electric vehicle adoption and whether a battery electric vehicle or plug-in hybrid electric vehicle (collectively PEVs) can fulfill the mission requirements.

Intertek acknowledges the support of Idaho National Laboratory, ICF International, and GCNP for participation in the study.

Intertek is pleased to provide this report and is encouraged by the enthusiasm and support from the National Park Service and GCNP personnel. 


\section{EXECUTIVE SUMMARY}

Federal agencies are mandated to purchase alternative fuel vehicles, increase consumption of alternative fuels, and reduce petroleum consumption. Available plug-in electric vehicles (PEVs) provide an attractive option in the selection of alternative fuel vehicles. PEVs, which consist of both battery electric vehicles (BEVs) and plug-in hybrid electric vehicles (PHEVs), have significant advantages over internal combustion engine (ICE) vehicles in terms of energy efficiency, reduced petroleum consumption, reduced production of greenhouse gas (GHG) emissions, and they provide performance benefits with quieter, smoother operation. This study intended to evaluate the extent to which Grand Canyon National Park (GCNP) could convert part or all of their fleet of vehicles from petroleum-fueled vehicles to PEVs.

It is likely that more fuel-efficient ICE vehicles, including hybrid electric vehicles, exist that could provide improvements for the current fleet; however, this study's focus is on replacing ICE vehicles with suitable PEVs.

BEVs provide the greatest benefit when it comes to fuel and emissions savings because all motive power is provided by the energy stored in the onboard battery pack. These vehicles use no petroleum and emit no pollutants at their point of use. PHEVs provide similar savings when their battery provides the motive power, but they also have the ability to extend their operating range with an onboard ICE. Because a PHEV can meet all transportation range needs, the adoption of a PHEV will be dependent on its ability to meet other transportation needs (such as cargo or passenger carrying). Operation of PHEVs in battery-only mode can be increased with opportunity charging at available charging stations; however, it should be noted that not all PHEVs have a mode in which the battery provides all motive power at all speeds. This study focuses on the mission requirements of the fleet of vehicles, with the objective of identifying vehicles that may be replaced with PEVs and with emphasis on BEVs that provide maximum benefit.

GCNP contains over 1,217,400 acres of land in northern Arizona. The climate is relatively mild with the South Rim's mean high/low temperatures at $63^{\circ} \mathrm{F} / 35^{\circ} \mathrm{F}$ and the North Rim's mean high/low temperatures at $56^{\circ} \mathrm{F} / 30^{\circ} \mathrm{F}^{\mathrm{a}}$. The geographic size of GCNP creates significant travel demands on its vehicle fleet and likewise provides opportunities for conversion of some vehicles to PEVs. GCNP identified 232 vehicles in its fleet, with11 of those vehicles being identified as representative of the fleet and instrumented for data collection and analysis. Fleet vehicle mission categories are defined in Section 4, and while the GCNP vehicles conduct many different missions, two missions (i.e., pool and support missions) were selected by agency management to be part of this fleet evaluation. These two mission categories accounted for 215 of the 232 total fleet vehicles. Thirteen enforcement vehicles were included in the GCNP vehicle information that may be considered for PEV replacement; however, none were monitored. The remaining four vehicles were specialty vehicles.

This report observes that a mix of BEVs and PHEVs are capable of performing most of the required missions and of providing an alternative vehicle

\footnotetext{
${ }^{\text {a }}$ Grand Canyon Park Profile 2013, http://www.nps.gov/grca/parkmgmt/upload/2013-park-profile.pdf [accessed June 30, 2014].
} 
for the pool and support vehicles, because while some vehicles travel long distances, the group could support some BEVs for the short trips and PHEVs for the longer trips. The recommended mix of vehicles will provide sufficient range for individual trips and time is available each day for charging to accommodate multiple trips per day. These charging events could occur at the vehicle home base. Replacement of vehicles in the current fleet would result in significant reductions in the emission of GHGs and in petroleum use, as well as reduced fleet operating costs.

PEVs that currently are commercially available cannot replace certain vehicles and missions, such as those requiring heavy-duty, load-hauling trucks and passenger vans; some of which were included in this study. However, based on the data collected from the monitored vehicles and extrapolating to the 232 vehicles, a fleet consisting of four specialty vehicles, four conventional heavyduty trucks, six conventional passenger vans, 80 BEVs and 138 PHEVs may meet the park's needs.

Electric power generation in the GCNP region relies heavily on nuclear energy as the fuel source. This results in lower GHG emissions than the national average. The replacement of 218 ICE vehicles with PEVs could result in an annual GHG savings over 1,454,610 $\mathrm{lb}-\mathrm{CO}_{2 \mathrm{e}}(69 \%$ reduction) and an annual fuel cost savings of over $\$ 296,044$ ( $80 \%$ reduction).

PEV charging stations could be located in various locations of GCNP and could benefit not only GCNP's own fleet vehicles but also those in the visiting public that own PEVs.

Intertek suggests that GCNP may wish to move forward in the near future with the replacement of pool and support vehicles with PEVs as current budget and vehicle replacement schedules allow. Certainly, the vehicle types studied in this report may be candidates for immediate replacement. 


\section{CONTENTS}

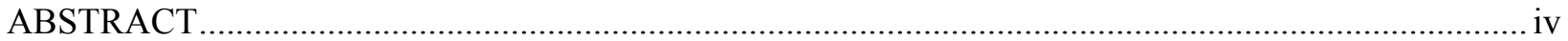

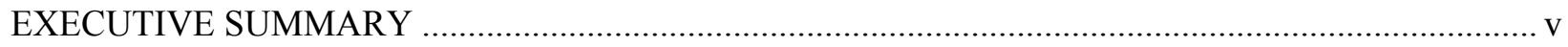

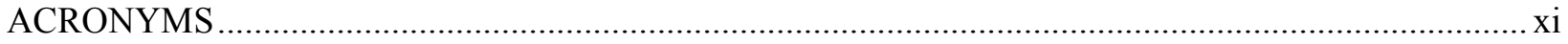

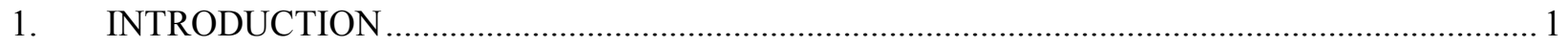

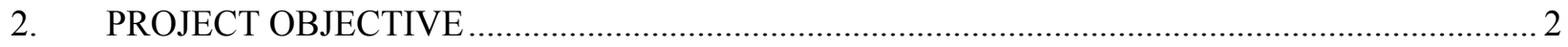

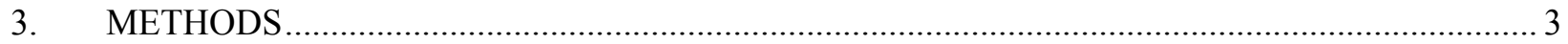

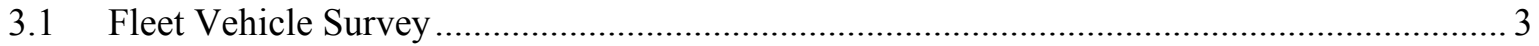

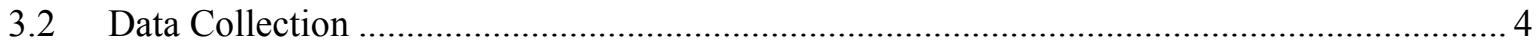

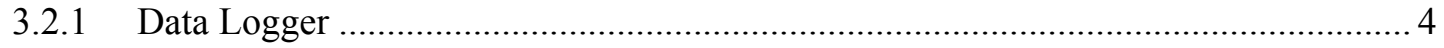

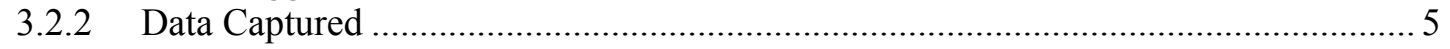

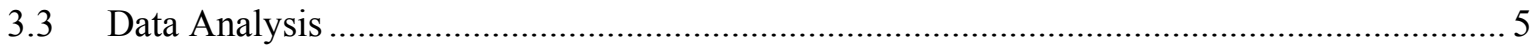

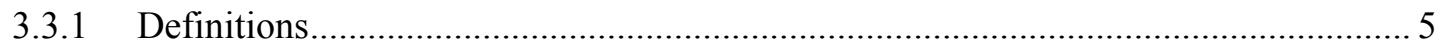

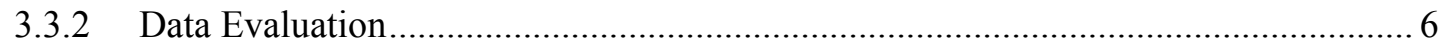

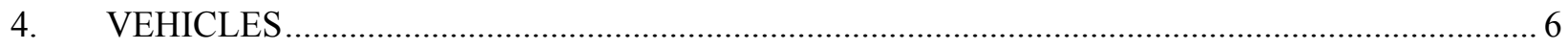

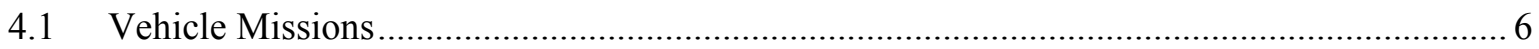

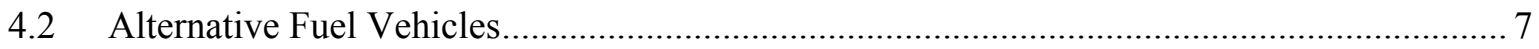

4.3 Battery Electric Vehicles and Plug-in Hybrid Electric Vehicles Benefits/Challenges ........... 8

4.3.1 Battery Electric Vehicle Benefits/Challenges ...................................................... 8

4.3.2 Plug-in Hybrid Electric Vehicle Benefits/Challenges............................................ 9

4.4 Plug-In Electric Vehicle Availability .......................................................................... 9

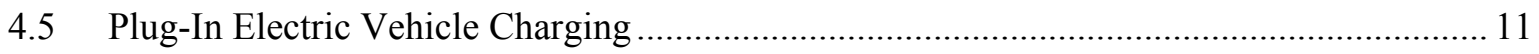

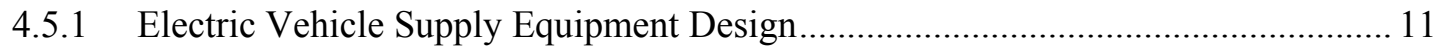

4.5.2 Electric Vehicle Supply Equipment Stations ........................................................ 14

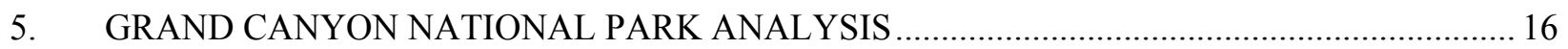

5.1 Grand Canyon National Park Fleet .............................................................................. 16

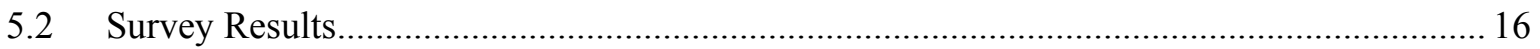

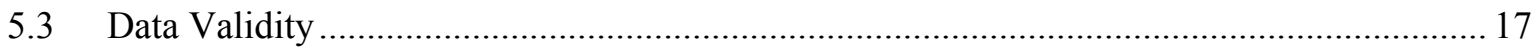




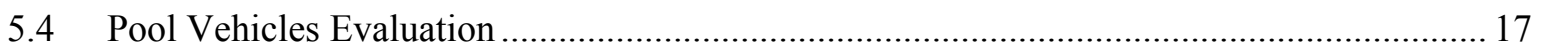

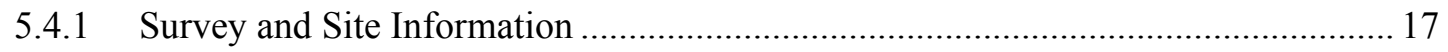

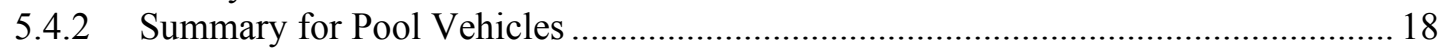

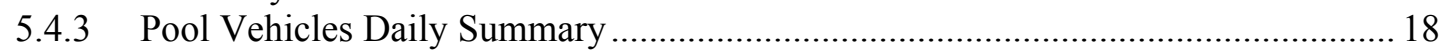

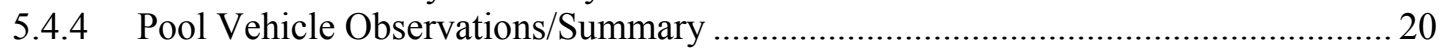

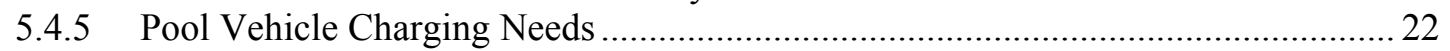

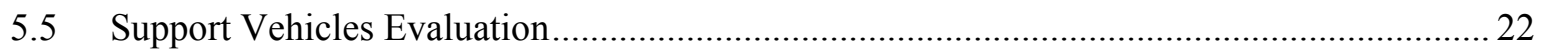

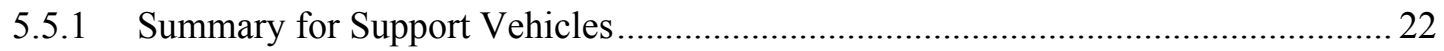

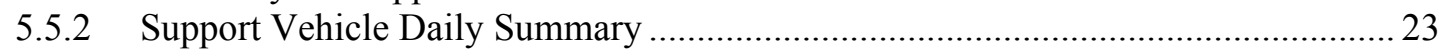

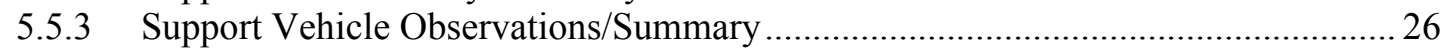

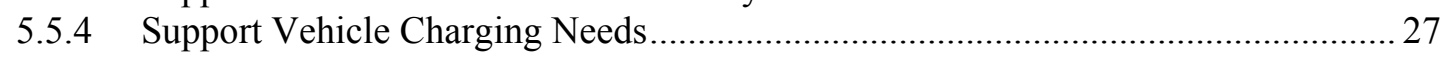

5.6 Balance of Fleet Vehicles ......................................................................................... 27

\section{GREENHOUSE GAS EMISSIONS AVOIDED AND FUEL COST REDUCTION}

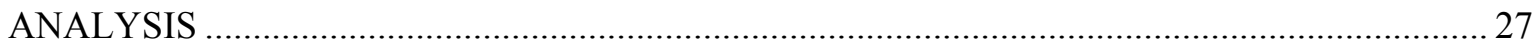

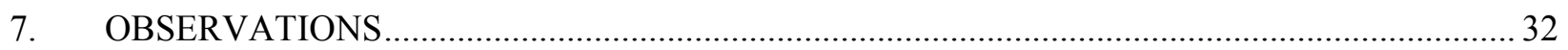

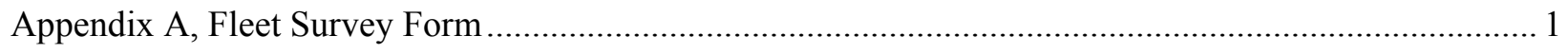

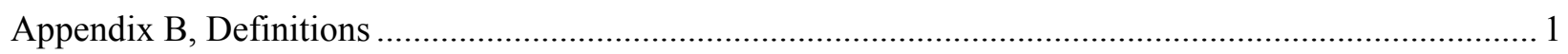

Appendix C, Grand Canyon National Park Map …..................................................................... 1

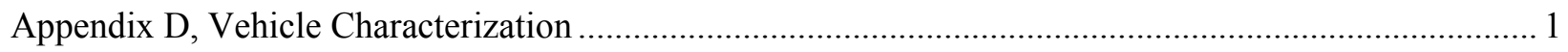

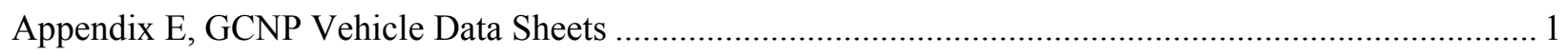

\section{FIGURES}

1. Grand Canyon National Park graphical representation .........................................................2

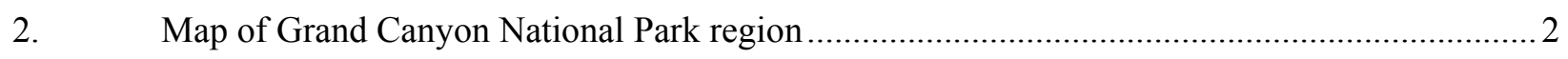

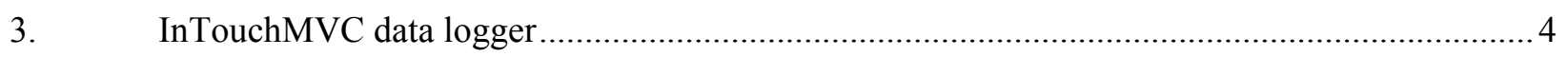

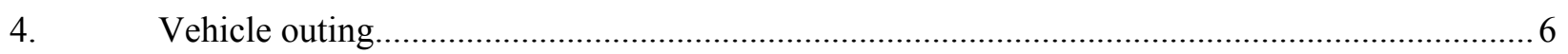

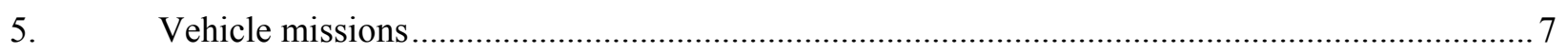

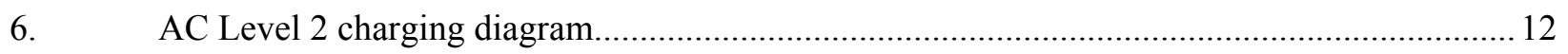

7. Society of Automotive Engineers charging configurations and ratings terminology ................ 13

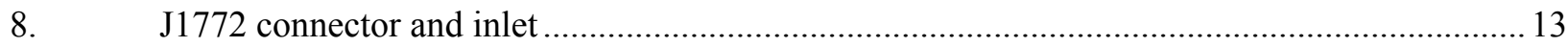




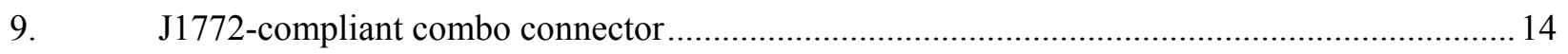

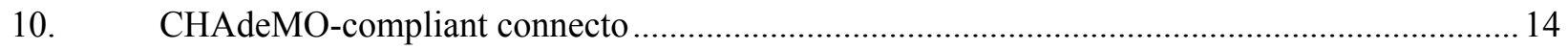

11. Public alternating current Level 2 electric vehicle support equipment................................... 15

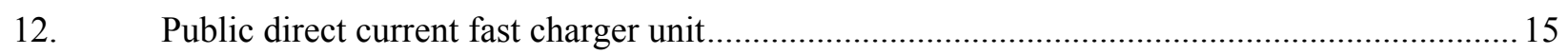

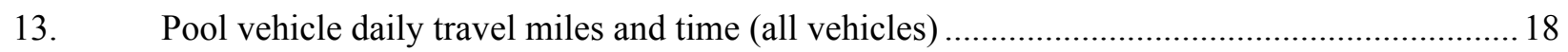

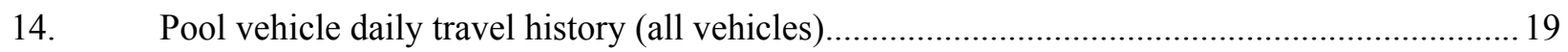

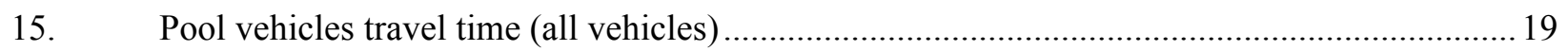

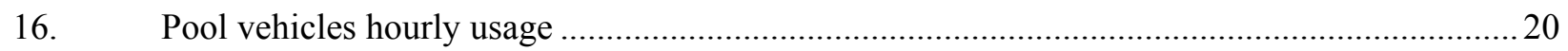

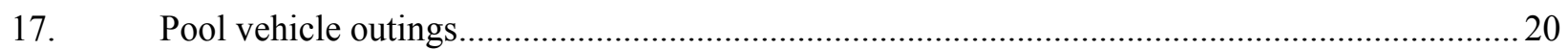

18. Public electric vehicle supply equipment in the Grand Canyon National Park region...............22

19. Support vehicles percentage of daily use versus daily travel miles and time (all

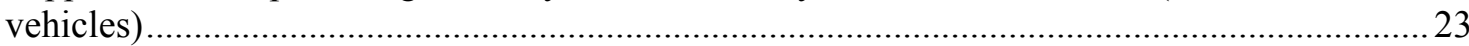

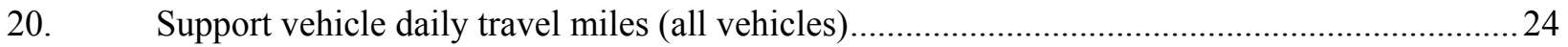

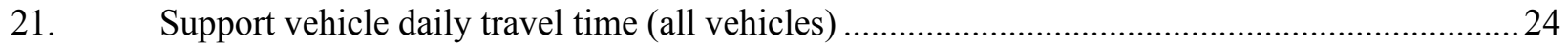

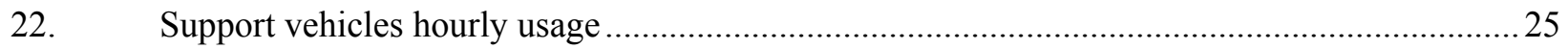

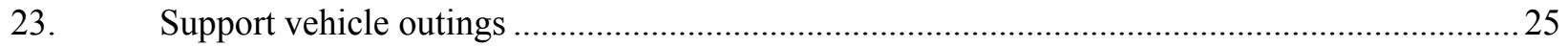

\section{TABLES}

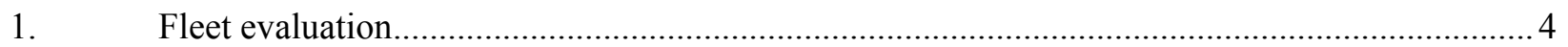

2. General Services Administration vehicle replacement requirements ....................................... 8

3. General Services Administration-certified plug-in electric vehicles ....................................... 10

4. Original equipment manufacturer plug-in hybrid electric vehicle cars and availability............ 10

5. Original equipment manufacturer battery electric vehicle cars and availability ...................... 10

6. Original equipment manufacturer plug-in hybrid electric vehicle trucks, vans, and

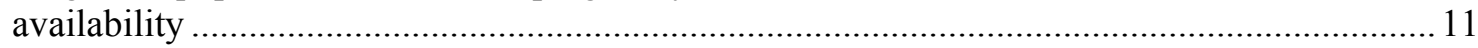

7. Original equipment manufacturer battery electric vehicle trucks, vans, and availability...........11

8. Grand Canyon National Park fleet vehicles........................................................................ 16 


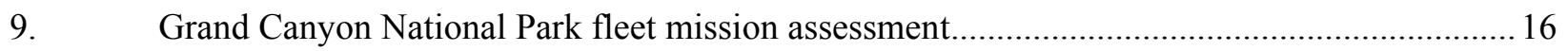

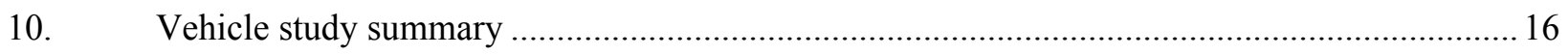

11. Detailed Grand Canyon National Park vehicle index ......................................................... 17

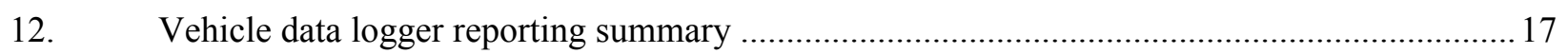

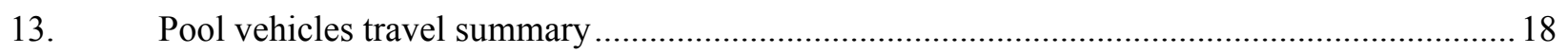

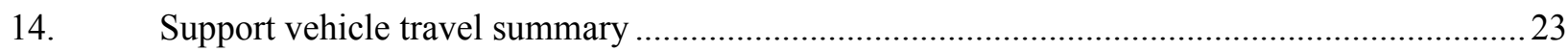

15. U.S. Environmental Protection Agency fuel economy ratings for current fleet vehicles ...........28

16. U.S. Environmental Protection Agency plug-in electric vehicle energy consumption

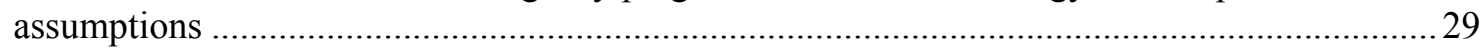

17. Plug-in electric vehicle substitutions for current vehicles ......................................................29

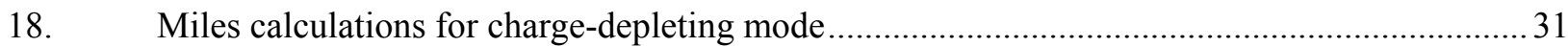

19. Greenhouse gas emissions avoidance and fuel cost reduction analysis summary....................31

20. Extrapolated greenhouse gas emissions avoided and fuel cost savings for the entire fleet ........ 32 


\section{ACRONYMS}

$\mathrm{AC} \quad$ alternating current

BEA Battelle Energy Alliance, LLC

BEV battery electric vehicle

CD charge depleting

CS charge sustaining

DC direct current

EPA U.S. Environmental Protection Agency

EVSE electric vehicle supply equipment

GCNP Grand Canyon National Park

GHG greenhouse gas emissions

GSA General Services Administration

ICE internal combustion engine

OEM original equipment manufacturers

PEV plug-in electric vehicle (includes BEVs and PHEVs, but not hybrid electric vehicles)

PHEV plug-in hybrid electric vehicle

SUV sport utility vehicle

VIN vehicle identification number 


\section{INTRODUCTION}

Federal agencies are mandated by the Energy Policy Act of $1992^{2}$, Energy Policy Act of $2005^{3}$, Executive Order 13423 (President Bush 2007), and the Energy Independence and Security Act of $2007^{5}$ to purchase alternative fuel vehicles, increase consumption of alternative fuels, and reduce petroleum consumption.

Battelle Energy Alliance, LLC (BEA), managing and operating contractor for Idaho National Laboratory, is the lead laboratory for the U.S. Department of Energy's advanced vehicle testing and manages the Advanced Vehicle Testing Activity's Federal Fleet Vehicle Data Logging and Characterization Study, which promotes utilization of advanced electric drive vehicle transportation technologies. The Advanced Vehicle Testing Activity focuses its testing activities on emerging and newly commercialized plug-in electric vehicle (PEV) technologies due to the high-energy efficiencies and reduced consumption of petroleum with the use of electric-drive vehicles. BEA selected Intertek Testing Services, North America (Intertek) to collect data on federal fleet operations and report the findings on vehicle and mission characterizations to support the successful introduction of PEVs into federal fleets.

It is likely that more fuel-efficient internal combustion engine (ICE) vehicles, including hybrid electric vehicles, exist that could provide improvements for the current fleet; however, this study's focus is on replacing ICE vehicles with suitable PEVs

Because of the large number of vehicles in federal fleets in the United States, these fleets provide a substantial opportunity for the introduction of battery electric vehicles (BEVs) and plug-in hybrid electric vehicles (PHEVs) (collectively, PEVs). However, to assess the scale of this opportunity, additional data are required to characterize the various missions performed by each fleet and to determine which existing vehicles are most suitable for replacement by a PEV.

The Grand Canyon National Park (GCNP), located in northern Arizona, contains over 1,217,400 acres of land (Figures 1 and 2 and Appendix C) ${ }^{6}$. Known for its scenic beauty, wilderness areas, trails, campgrounds, and varied recreational uses, the park receives approximately 4.5 million site visitors a year. ${ }^{7}$ Appendix C provides a detailed map of GCNP, identifying several key locations.

The climate is relatively mild, with the South Rim's mean high/low temperatures at $63^{\circ} \mathrm{F} / 35^{\circ} \mathrm{F}$ and the North Rim's mean high/low temperatures at $56^{\circ} \mathrm{F} / 30^{\circ} \mathrm{F} .{ }^{8} \mathrm{GCNP}$ is an excellent site for fleet evaluation, not only due to its size, diversity of terrain, diversity of weather, and vehicle types, but because of its accessibility by the public. GCNP has an opportunity to be a leader in the adoption of BEVs and PHEVs for its fleet.

\footnotetext{
${ }^{2}$ http://thomas.loc.gov/cgi-bin/query/z?c102:h.r.776.enr [accessed January 10, 2014].

${ }^{3}$ http://www.gpo.gov/fdsys/pkg/BILLS-109hr6enr/pdf/BILLS-109hr6enr.pdf [accessed January 10, 2014].

${ }^{4}$ http://www.gsa.gov/portal/content/102452 [accessed January 10, 2014].

${ }^{5}$ http://www.gpo.gov/fdsys/pkg/PLAW-110publ140/pdf/PLAW-110publ140.pdf [accessed January 10, 2014].

${ }^{6}$ http://www.nps.gov/grca/index.htm [accessed July 22, 2014].

${ }^{7}$ https://irma.nps.gov/Stats/SSRSReports/Park\%20Specific\%20Reports/Annual\%20Park\%20Recreation\%20Visitation\%20Graph \%20(1904\%20-\%20Last \%20Calendar\%20Year)?Park=grca [Accessed June 3, 2014].

${ }^{8}$ Grand Canyon Park Profile 2013 http://www.nps.gov/grca/parkmgmt/upload/2013-park-profile.pdf [accessed June 30, 2014].
} 


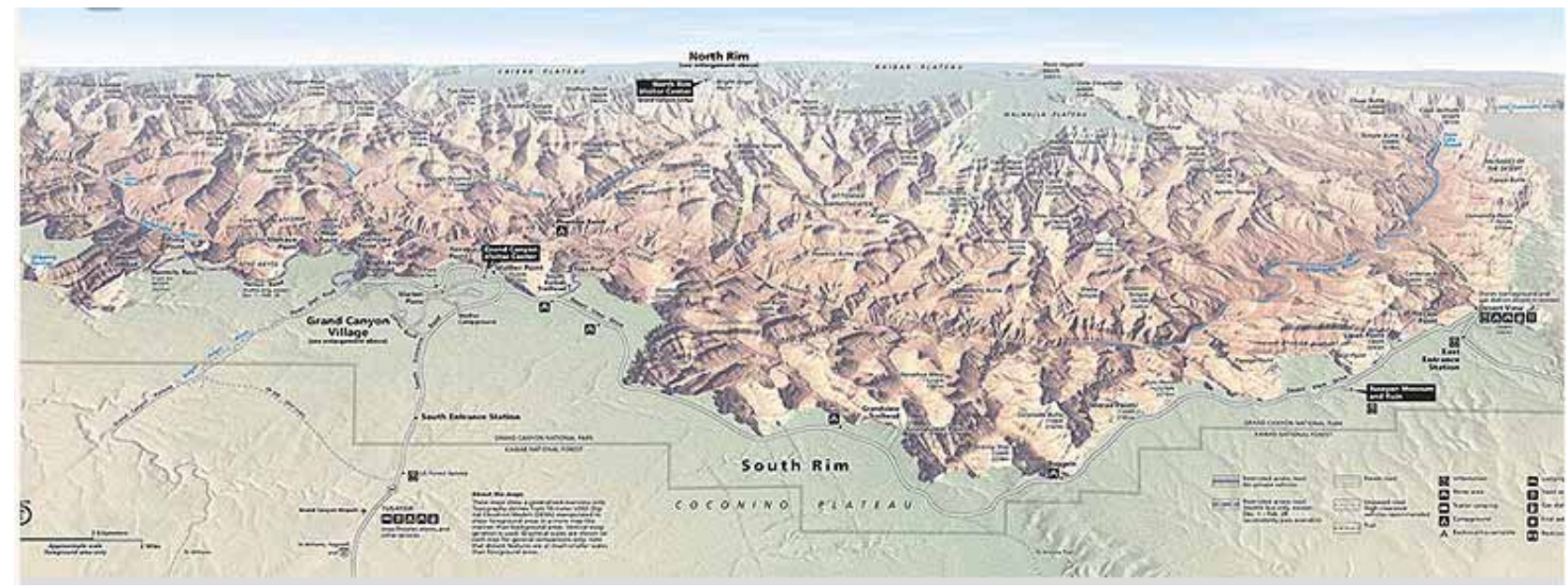

Figure 1. Grand Canyon National Park graphical representation.

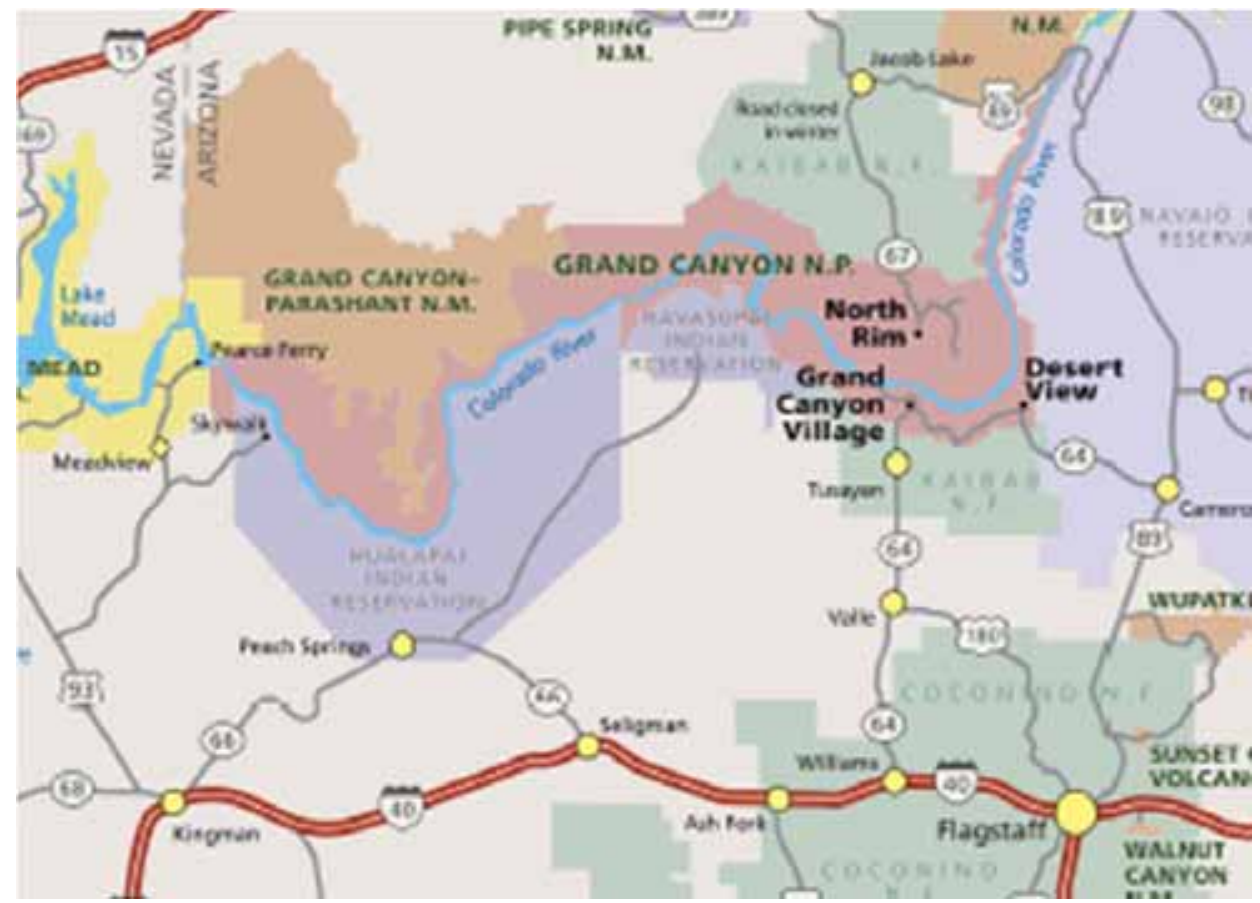

Figure 2. Map of Grand Canyon National Park region.

\section{PROJECT OBJECTIVE}

This study explores federal fleet vehicles and their usage characteristics, with a primary objective of supporting the goals of Presidential Executive Order 13514, which includes the following:

- Pursuing opportunities with vendors and contractors to address and incentivize greenhouse gas (GHG) emission reductions and petroleum use reductions

- Implementing strategies and accommodations for transit, travel, training, and conferences that actively reduce carbon emissions associated with commuting and travel by agency staff

- Meeting GHG emissions reductions associated with other federal government sustainability goals 
- Implementing innovative policies and practices that address agency-specific Scope 3 GHG emissions. ${ }^{9}$

Because of the large number of vehicles in the federal fleets, there is a substantial opportunity for PHEV and BEV adoption. Federal fleets offer an opportunity as a first market replacement for alternative fuels due to their scale, refueling patterns, and regular vehicle turnover. ${ }^{10}$

This project has the following four defined tasks:

1. Data collection: Coordinate with the fleet manager to collect data on agency fleet vehicles. This includes collecting information on the fleet vehicles and installing data loggers on a representative sample of the fleet vehicles to characterize their missions.

2. Data analysis and review: Examine the data collected by the loggers and fleet vehicle characteristics to describe typical fleet activity. Incorporate fleet manager's input on introducing PEVs to the agency's fleet.

3. PEV implementation feedback: Provide feedback to fleet personnel and BEA on the selection criteria for replacement PEVs in their specific fleet vehicle missions.

4. Observations and recommendations: Provide actionable information to introduce PEVs into agency fleet operations and assess any related impacts for the facility.

Data collected from vehicles include trip distance, idle time, time between uses, and stop locations. Data collection continues for 30 to 60 days using a non-intrusive data logger, which gathers and transmits information using global positioning satellites and cellular service. The loggers collect data at 1-minute intervals and transmit when an active signal is present.

Extrapolating the results of this analysis to the larger fleet provides estimates of potential savings in gasoline consumption and GHG emissions. This report also provides recommendations relating to fleet management of BEVs and PHEVs for additional consideration.

Fleet managers may use the information supplied in this report to help them to identify which vehicles are candidates for replacement by BEVs or PHEVs based on their use. BEVs are preferred because of the greater potential reduction of GHG emissions, fuel costs and petroleum usage, but they are not likely to be suitable for all vehicle missions.

The information in this report supports a final report to BEA/Idaho National Laboratory and the U.S. Department of Energy. The aggregated results for all agencies' fleets will provide an overview of federal fleets, vehicle missions, vehicle uses, and agencies needs to plan and establish a more systematic method for the adoption of BEVs and PHEVs.

\section{METHODS}

\subsection{Fleet Vehicle Survey}

Agency fleet managers selected fleet vehicles for this study and provided basic information for each vehicle, including its managing agency, home base for the vehicle, contact information, primary vehicle mission, vehicle ownership, fuel type, and annual mileage driven. This information was collected using the vehicle information form shown in Appendix A.

GCNP identified 232 fleet vehicles in their interior and General Services Administration (GSA) fleet. Vehicle missions were assumed based on the GCNP input shown in Table 1. (Note that Section 4

\footnotetext{
${ }^{9}$ http://energy.gov/sites/prod/files/2013/10/f3/eo13514.pdf [accessed February 5, 2014].

${ }^{10}$ Fleet Purchase Behavior: Decision Processes and Implications for New Vehicle Technologies and Fuel, Nesbitt, Sperling, University of California, Davis 2001.
} 
provides descriptions of the vehicle mission types.) Intertek coordinated with the GCNP fleet manager to identify the specific vehicles for data collection for inclusion in the study. The fleet manager assessed their wide range of vehicles and made selections of high-interest, representative vehicles based on vehicle missions and vehicle type/class. Selection also favored vehicles used at least twice a week. Because data loggers rely on the vehicle's battery power, non-use of the vehicle can result in the vehicle having a depleted battery. Intertek received no reports of depleted batteries during the study at GCNP. Eleven vehicles were selected, with five being pool vehicles and six being support vehicles.

Table 1. Fleet evaluation.

\begin{tabular}{lccc}
\multicolumn{1}{c}{ Vehicle Mission } & Study Vehicles & $\begin{array}{c}\text { Total Fleet } \\
\text { Reported }\end{array}$ & Percentage Studied \\
\hline Pool Vehicles & 5 & 106 & $4.7 \%$ \\
Support Vehicles & 6 & 109 & $5.5 \%$ \\
Enforcement Vehicles & & 13 & $0 \%$ \\
Specialty Vehicles & 11 & 16 & $0 \%$ \\
Total Fleet Vehicles & & 232 & $4.7 \%$
\end{tabular}

\subsection{Data Collection}

Individual privacy concerns exist when monitoring vehicle movement with data loggers. Data collection occurs by vehicle identification, which is identified by Intertek, data logger number, and vehicle identification number (VIN), or an agency-assigned vehicle number. Intertek receives no information related to the vehicle operator and provides no raw data to the fleet managers. In this manner, Intertek does not collect, analyze, or report on individual driving habits.

\subsubsection{Data Logger}

Non-intrusive data loggers, produced by InTouchMVC ${ }^{11}$ and depicted in Figure 3, were inserted into the vehicle's onboard diagnostic port to collect and transmit the relevant data. Installation of the data logger and the manual recording of information about the vehicle, which ties the logger and vehicle together in the data, typically takes less than 5 minutes. Once installed and activated (during vehicle use), the data loggers collect vehicle information once every minute during vehicle operation and transmit by cellular communication to the data center.

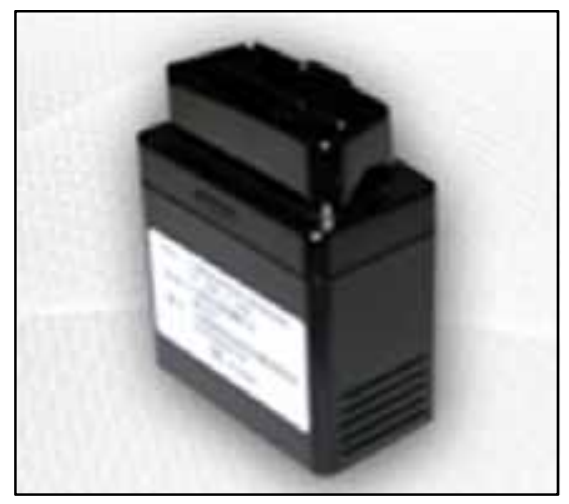

Figure 3. InTouchMVC data logger.

${ }^{11}$ www.intouchmvc.com [accessed January 10, 2014]. 
Intertek maintains the data logger's connectivity and verifies data transmission weekly. Missing data (reported as "null" values) are frequently the result of lost global positioning system reception, logger device removal, or extended periods in regions with insufficient cellular reception. Intertek filters the vehicle and data logger information if these null values present a significant impact on the data collected and no resolution is possible. This report also identifies the statistics on this validation process.

GCNP requested and installed 11 data loggers into the selected fleet vehicles. The agency removed and shipped the data loggers to Intertek at the conclusion of the data collection period.

\subsubsection{Data Captured}

Data consist of key-on events, key-off events, and position updates logged every minute while the vehicle is keyed-on. InTouchMVC converted these data points into records of trip events, stop events, and idle events.

From these data points, the following information was available for evaluation:

- Trip start and stop time and location

- Trip distance and duration

- Idle start time, location, and duration

- Stop start time, location, and duration.

\subsection{Data Analysis}

\subsubsection{Definitions}

Figure 4 illustrates a vehicle outing, which is comprised of trips, stops, and idle events, that may occur during one day or over several days. The following list provides a definition of these terms:

1. Outing: An outing is the combination of trips and stops that begin at the home base and includes all travel until the vehicle returns home.

2. Trip: A trip begins with a key-on event and ends with the next key-off event.

3. Vehicle stop: A vehicle stop includes a key-off/key-on event pair.

4. Idle time: Idle time is the amount of time a vehicle spends stationary after a key-on event when the vehicle is not moving for a period of 3 minutes or longer.

5. Trip travel time: Trip travel time is the amount of time required to complete a trip, excluding stops, but including idle time.

Definitions of additional analysis and survey terms are as follows:

1. Operating shift: Fleet manager-defined period worked.

2. Study days: Days during which the data loggers are connected.

3. Vehicle days: Study days during which a vehicle is used.

4. Null values: Data record unusable for analysis for various reasons. 


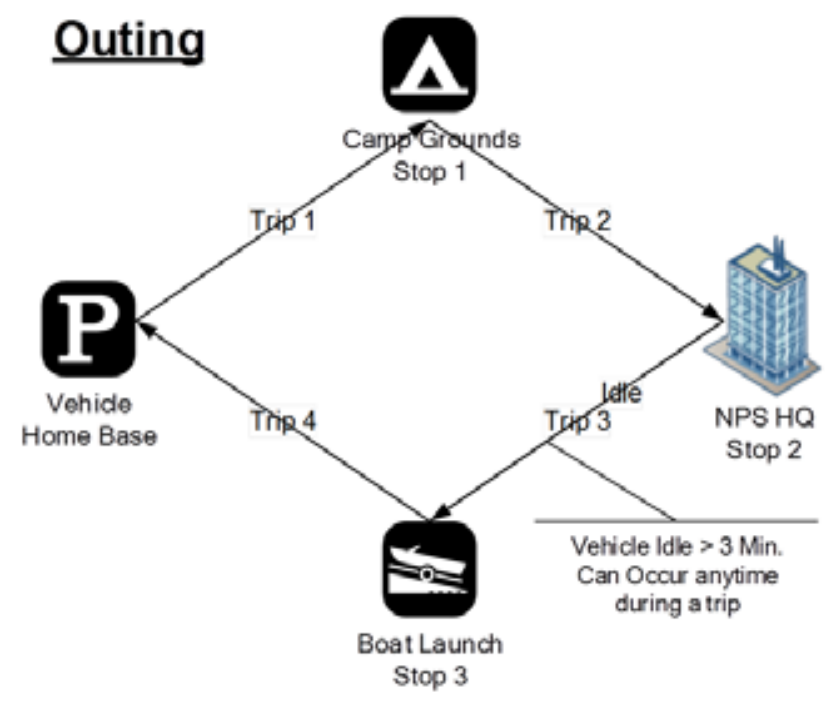

Figure 4. Vehicle outing.

\subsubsection{Data Evaluation}

Processing the data involves removal of null values and aggregation by different spatial and temporal scales. Aggregation was by day, by trip, and by outing to produce figures showing the patterns of use. Aggregation by vehicle mission followed to characterize use for the agency fleet. Section 5 presents these results. Data were extrapolated to provide the overall fleet usage and benefit analysis when fleet information was provided. Section 6 presents these benefits. Intertek observations are included in Section 7.

Statistical data analysis uses Python 2.7 with the MATLAB Plotting Library graphics environment (Matplotlib) and spatial display with ESRI ArcGIS. ${ }^{12}$ Frequency distributions summarize travel behavior of each vehicle and vehicle mission during the study period. Rounding of the tables and figures are to three significant digits.

\section{VEHICLES}

\subsection{Vehicle Missions}

The vehicle mission is an important characteristic in the fleet study. Information used to define the vehicle mission includes the vehicle's configuration, vehicle use, classification per 40 CFR Part 600.31582 and the Environmental Protection Agency (EPA), the participating agency's use, and generally assumed vehicle use. Based on the fleet information gathered, Intertek has established the following seven mission/vehicle categories for analysis. They are listed as follows and examples are depicted in Figure 5:

1. Pool vehicles: A pool vehicle is any automobile (other than the low-speed vehicles identified below) manufactured primarily for use in passenger transportation, with not more than 10 passengers.

2. Enforcement vehicles: Vehicles specifically approved in an agency's appropriation act for use in apprehension, surveillance, police, or other law enforcement work. This category also includes site security vehicles, parking enforcement, and general use, but the vehicles are capable of requirements to support enforcement activities. Appendix C provides further definition.

\footnotetext{
${ }^{12}$ www.esri.com [accessed January 10, 2014].
} 
3. Support vehicles: Vehicles assigned to a specific work function or group to support the mission of that group. Vehicles are generally passenger vehicles or light-duty pickup trucks and may contain after-market modifications to support the mission.

4. Transport vehicles: Light, medium, or heavy-duty trucks used to transport an operator and tools or equipment of a non-specific design or nature. The vehicle's uses include repair, maintenance, or delivery.

5. Specialty vehicles: Vehicles designed to accommodate a specific purpose or mission (such as ambulances, mobile cranes, and handicap controls).

6. Shuttles/buses: Vehicles designed to carry more than 12 passengers and further outlined in 49 CFR 532.2.

7. Low-speed vehicle: Vehicles that are legally limited to roads with posted speed limits up to $45 \mathrm{mph}$ and that have a limited load-carrying capability.

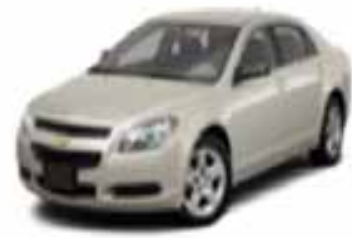

Pool Vehicle

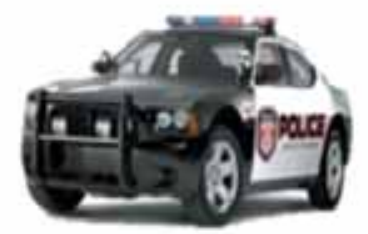

Enforcement Vehicle

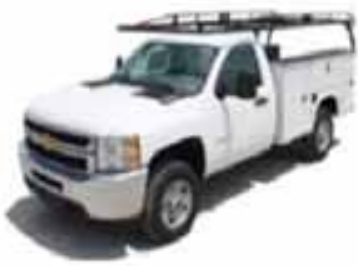

Support Vehicle

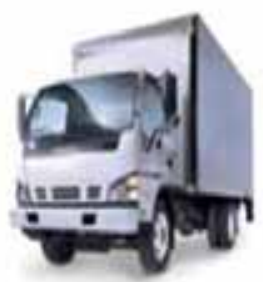

Transport Vehicle

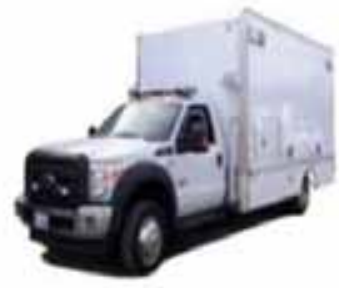

Specialty Vehicle

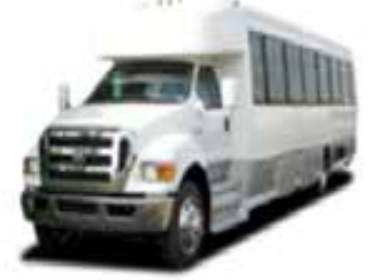

Shuttle / Bus

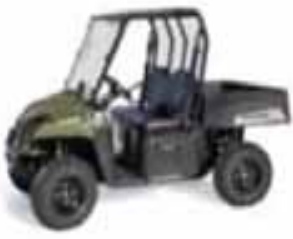

Low Speed

Vehicles

Figure 5. Vehicle missions.

\subsection{Alternative Fuel Vehicles}

As the operating agency, GCNP has a unique opportunity to plan for the adoption of BEVs and PHEVs, along with planning for supporting infrastructure. The adoption of PHEVs and BEVs is a primary goal of GSA and it supports the directives previously referenced.

As GSA increases its certification of PHEVs and BEVs, agencies can plan for vehicle replacement through GSA for passenger vehicles and trucks. Table 2 presents the replacement requirements for fleet vehicles. Note that both of the age and mileage requirements need to be met in order for the vehicle to qualify for replacement, except where noted as "or". 


\subsection{Battery Electric Vehicles and Plug-in Hybrid Electric Vehicles Benefits/Challenges}

BEVs are fully powered by the battery energy storage system available onboard the vehicle. The Nissan Leaf is an example of a BEV. Because the BEV has no other energy source for propulsion, the range, power requirements, and mission of the needed vehicle factor greatly in purchasing decisions. Maximizing BEV capabilities typically requires batteries more than an order of magnitude larger in capacity than the batteries in hybrid electric vehicles.

Table 2. General Services Administration vehicle replacement requirements.

\begin{tabular}{cccc}
\hline & GSA Vehicle Replacement Requirements & \\
& Fuel Type & Years & Miles \\
\hline Passenger vehicles & Gasoline or & 3 & 36,000 \\
& alternative fuel & 4 & 24,000 \\
& vehicle & 5 & Any mileage \\
& Hybrid & Any age & 75,000 \\
Light trucks 4 x 2 & Non-diesel & 5 & Any miles \\
& Diesel & 6 & Any miles \\
& Hybrid & 7 & 65,000 \\
Light trucks 4 x 4 & Non-diesel & 7 & 150,000 \\
& Diesel & 7 or & Any mileage \\
& Hybrid & 8 or & 60,000 \\
& & 7 & 150,000 \\
\hline
\end{tabular}

PHEVs obtain their power from at least two energy sources. The typical PHEV configuration uses a battery and an ICE that is powered by either gasoline or diesel. PHEV designs differ between manufacturers. All have a charge-depleting (CD) mode, in which the battery is depleted of its stored energy to propel the vehicle, and a charge-sustaining (CS) mode (or extended range mode) that is entered after CD mode is complete and in which the battery and the ICE work together to provide propulsion, while the state of charge of the battery is maintained between set limits. Some PHEVs' operation in CD modes is purely electric, while others employ the engine to supplement the battery power during the initial battery depletion to a set state of charge (usually below 50\%).

\subsubsection{Battery Electric Vehicle Benefits/Challenges}

EPA identifies the following benefits of BEVs ${ }^{14}$ :

- Energy efficient: Electric vehicles convert about 59 to $62 \%$ of the electrical energy from the grid to power at the wheels, whereas conventional gasoline vehicles only convert about 17 to $21 \%$ of the energy stored in gasoline to power at the wheels.

- Environmentally friendly: PEVs emit no tailpipe pollutants, although the power plant producing the electricity may emit them. Electricity from nuclear, hydro, solar, or wind-powered plants causes no air pollutants.

\footnotetext{
${ }^{13}$ http://www.gsa.gov/graphics/fas/VehicleReplacementStandardsJune2011Redux.pdf [accessed January 10, 2014].

${ }^{14} \mathrm{http} / / / w w w . f u e l e c o n o m y . g o v / f e g /$ evtech.shtml [accessed December 27, 2013].
} 
- Performance benefits: Electric motors provide quiet, smooth operation and exhibit maximum torque at zero and low speeds, while also requiring less maintenance than ICEs.

- Reduce energy dependence: Electricity is a domestic energy source.

EPA also identifies challenges associated with BEVs, including the following:

- Driving range: Most BEVs can only travel about 100 to 200 miles (or less) before recharging, whereas gasoline vehicles can often travel over 300 miles before refueling and some much further.

- Recharge time: Fully recharging the battery pack can take 4 to 8 hours. With a high-power direct current (DC) fast charger, restoration from a depleted state to $80 \%$ capacity can take approximately 30 minutes.

- Battery cost: The large battery packs are expensive and may need to be replaced one or more times.

- Bulk and weight: Battery packs are heavy and take up considerable vehicle space.

\subsubsection{Plug-in Hybrid Electric Vehicle Benefits/Challenges}

EPA identifies the following benefits of PHEVs ${ }^{15}$ :

- Less petroleum use: PHEVs are expected to use about 40 to $60 \%$ less petroleum than conventional vehicles. Because electricity is produced primarily from domestic resources, PHEVs reduce dependence on oil.

- Fewer emissions: PHEVs are expected to emit fewer GHG emissions than conventional vehicles, but as with BEVs, the difference depends largely on the type of power plant supplying the electricity.

- Higher vehicle costs, lower fuel costs: PHEVs will likely cost $\$ 1,000$ to $\$ 7,000$ more than comparable non-PHEVs. Fuel will cost less because electricity is much cheaper than gasoline, but fuel savings depend on how much of the driving is done with the off-board electrical energy.

- Recharging takes time: Recharging the battery typically takes several hours. However, PHEVs do not have to be plugged in to be driven. They can be fueled solely with gasoline, but will not achieve maximum range, fuel economy, or fuel savings without charging.

- Measuring fuel economy: Because a PHEV can operate on electricity alone, gasoline alone, or a mixture of the two, EPA provides a fuel economy estimate for gasoline-only operation (CS mode), electric-only operation (all-electric CD mode), or combined gasoline and electric operation (blended CD mode).

In most cases, the PEV retail cost is higher than a non-PEV model. This incremental purchase cost may be a fleet budget challenge; however, many original equipment manufacturers (OEMs) have offered incentives to encourage the use and adoption of BEVs and PHEVs. Some OEMs have recently reduced the vehicle cost, while also increasing vehicle range. Additionally, federal and state incentives have increased the attractiveness of purchasing a PEV. A common assumption is that increasing PEV sales will result in a reduction in this incremental purchase cost and a positive feedback loop will ensue.

\subsection{Plug-In Electric Vehicle Availability}

GSA provides a summary of light and medium-duty passenger vehicles available for lease or purchase through the GSA portal ${ }^{16}$; however, not all BEVs and PHEVs currently on the market are certified to be GSA replacements. Vehicles not on the GSA list of certified vehicles require an agency to self-certify a functional need or provide alternative measures for exemptions. Table 3 summarizes the

${ }^{15}$ http://www.fueleconomy.gov/feg/phevtech.shtml [accessed July 19, 2013]

${ }^{16} \mathrm{http} / / / w w w . g s a . g o v / p o r t a l / c o n t e n t / 104224$ [accessed March 6, 2014] 
vehicles that may be suitable replacements and are certified replacements through GSA. Note that the $\mathrm{CD} / \mathrm{CS}$ column provides the EPA fuel economy values for CD and CS modes. The fuel economy of CD mode is provided in units of miles-per-gallon-of-gasoline-equivalent (MPGe). This metric allows for electricity consumption during CD mode to be compared with fuel consumption during CS mode (or against conventional vehicles). The Nissan Leaf and Mitsubishi i-MiEV are not included in the alternative fuel guide for 2014, but they have appeared in previous guides.

Replacement is dependent on vehicle configuration characteristics and the vehicle mission. Further evaluation related to vehicle purpose and mission follows in Section 5.

Tables 4 through 7 provide summaries of PHEVs and BEVs either currently available or near commercialization in both passenger cars and pickup trucks, but do not appear on the GSA certified vehicle list. These vehicles may qualify for use by the agency through demonstrating a functional need.

Table 3. General Services Administration-certified plug-in electric vehicles.

\begin{tabular}{lcccc}
\multicolumn{1}{c}{ Make/Model } & \multicolumn{1}{c}{ GSA Class } & Type & CD/CS & GSA Incremental Price \\
\hline Chevrolet Volt & Sedan, Subcompact & PHEV & $98 \mathrm{MPGe} / 37 \mathrm{mpg}$ & $\$ 17,087.18$ \\
Ford C-MAX & Sedan, Subcompact & PHEV & $100 \mathrm{MPGe} / 38 \mathrm{mpg}$ & $\$ 14,899.52$ \\
Energi & & & \\
Ford Focus Electric & Sedan, Subcompact & BEV & $110 \mathrm{MPGe} / 99 \mathrm{mpg}$ & $\$ 16,573.09$ \\
Ford Fusion Energi & Sedan, Compact & PHEV & $100 \mathrm{MPGe} / 38 \mathrm{mpg}$ & $\$ 19,289.99$ \\
\hline
\end{tabular}

Note that EPA differs in vehicle class. EPA identifies the Volt as a compact, the C_MAX Energi as a midsize, the Fusion Energi as a midsize, and the Focus as a compact. ${ }^{17}$

Table 4. Original equipment manufacturer plug-in hybrid electric vehicle cars and availability.

\begin{tabular}{llc}
\multicolumn{1}{c}{ Make } & \multicolumn{1}{c}{ Model } & Model Year \\
\hline Audi & A3 eTron PHEV & 2015 (estimate) \\
Chevrolet & Volt & 2011 \\
Honda & Accord PHEV & 2014 \\
Toyota & Prius PHEV & 2012 \\
Volvo & V60 Plug-in & 2016 (estimate) \\
BMW & i3 with range extender & 2014 \\
\hline
\end{tabular}

Table 5. Original equipment manufacturer battery electric vehicle cars and availability.

\begin{tabular}{llc}
\multicolumn{1}{c}{ Make } & \multicolumn{1}{c}{ Model } & Model Year \\
\hline BMW & i3 & 2014 \\
Chevrolet & Spark EV & 2014 \\
Fiat & 500e & 2013 \\
Ford & Focus Electric & 2012 \\
Honda & Fit EV & 2013 \\
Kia & Soul EV & 2014 (estimate) \\
Mercedes-Benz & B-Class ED & 2015 (estimate) \\
Nissan & Leaf & 2011 \\
\hline
\end{tabular}

${ }^{17} \mathrm{http}: / /$ www.fueleconomy.gov/feg/Find.do?action=sbs\&id=34130 [accessed August 1, 2014]. 


\begin{tabular}{llc}
\multicolumn{1}{c}{ Make } & \multicolumn{1}{c}{ Model } & Model Year \\
\hline smart & ED & 2014 \\
Tesla & Model S & 2012 \\
Tesla & Model X & 2015 (estimate) \\
Volkswagen & Golf & 2015 (estimate) \\
Volvo & C30 Electric & 2016 (estimate) \\
\hline
\end{tabular}

Table 6. Original equipment manufacturer plug-in hybrid electric vehicle trucks, vans, and availability.

\begin{tabular}{llc}
\multicolumn{1}{c}{ Make } & \multicolumn{1}{c}{ Model } & Model Year \\
\hline Land Rover & Range Rover Sport & 2016 (estimate) \\
Mitsubishi & Outlander PHEV & 2015 (estimate) \\
Via & VTRUX VR300 & 2013 \\
\hline
\end{tabular}

Table 7. Original equipment manufacturer battery electric vehicle trucks, vans, and availability.

\begin{tabular}{clc} 
Make & \multicolumn{1}{c}{ Model } & Model Year \\
\hline Nissan & eNV200 & 2015 (estimate) \\
Toyota & RAV4 EV & 2013 (California only - \\
& & elsewhere 2015 estimate) \\
\hline
\end{tabular}

As further indication of the expanding market for PEVs, companies are offering after-market vehicle upgrades involving the addition of plug-in capabilities to OEM vehicles. For example, Echo Automotive headquartered in Scottsdale, Arizona is a newly formed company that, in 2012, began to offer a "...lowcost, bolt-on, plug-in hybrid system that can quickly be installed on new or existing fleet vehicles to increase fuel efficiency and decrease operating costs - all without affecting the OEM power train or requiring costly infrastructure." ${ }^{18}$ Options, such as this company's conversions, might be of benefit to the passenger vans identified in the GCNP fleet but for which no replacement PEV is currently available.

\subsection{Plug-In Electric Vehicle Charging}

Refueling electric vehicles presents some challenges and some opportunities not encountered when refueling petroleum-fueled vehicles. Recharging the battery of a PHEV follows the same methodology as that for BEVs. This section provides basic information on recharging PEVs.

\subsubsection{Electric Vehicle Supply Equipment Design}

4.5.1.1 Charging Components. Electric vehicle supply equipment (EVSE) stations deliver electric power from the utility to the applicable charge port on the vehicle. Figure 6 illustrates the primary components of a typical EVSE unit (Figure 6 shows an alternating current (AC) Level 2).

The electric utility delivers AC current to the charging location. The conversion from AC to DC electricity that is necessary for battery charging can occur either on or off-board the vehicle. Section 4.5.1.2 provides further explanation of the different EVSE configurations. For onboard conversion, AC current flows through the PEV inlet to the onboard charger. The charger converts AC to the DC current required to charge the battery. A connector attached to the EVSE inserts into a PEV inlet to establish an electrical connection to the PEV for charging and information/data exchange. Off-board conversion, also known as DC charging, proceeds in a similar manner, except that the AC to DC

\footnotetext{
${ }^{18}$ http://www.echoautomotive.com/index.php?option=com_content\&view=article\&id=8 [accessed July 14, 2014].
} 
conversion occurs in a charger that is off board the vehicle and, thus, bypasses any onboard charger. For both AC and DC charging, the PEV's battery management system on board the vehicle controls the battery rate of charge, among other functions. All current PEVs have an onboard charger; some BEVs (but no PHEVs currently) accommodate DC charging.

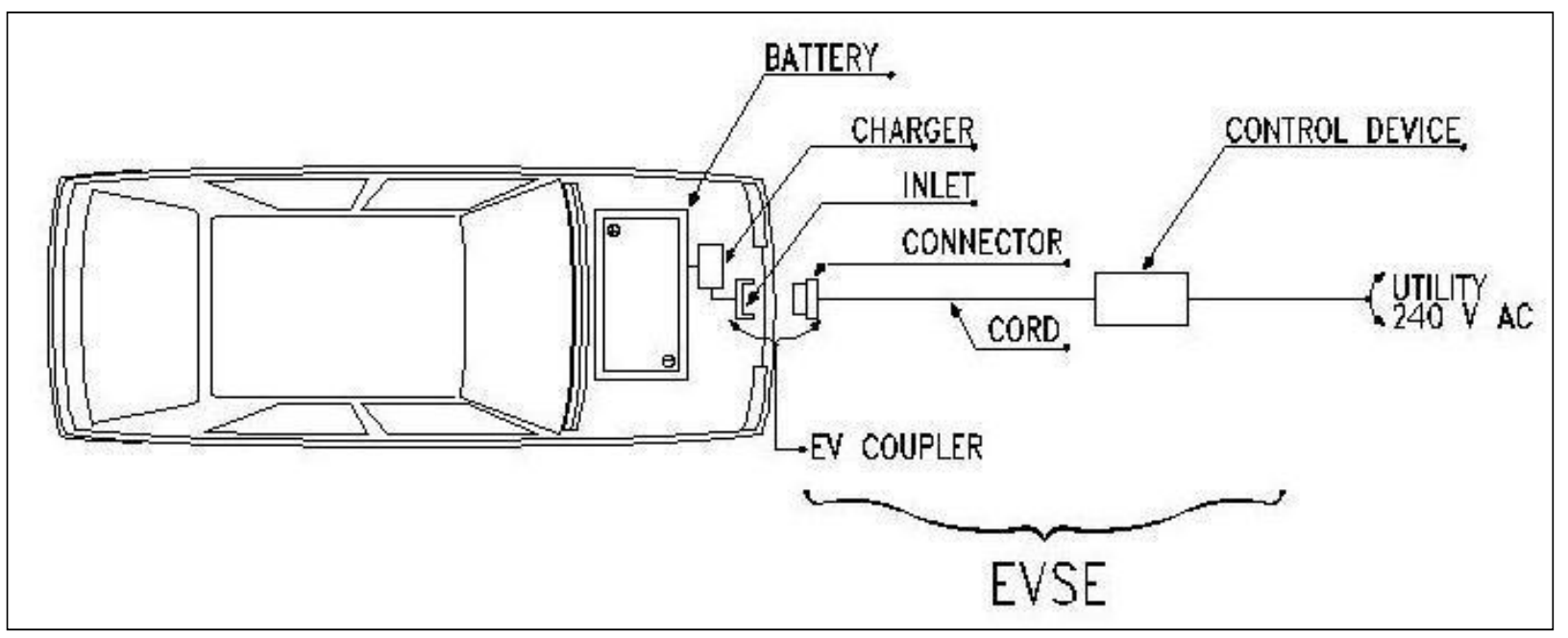

Figure 6. AC Level 2 charging diagram ${ }^{19}$.

4.5.1.2 Charging Configurations and Ratings. The Society of Automotive Engineers standardized the requirements, configurations, and equipment followed by most PEV suppliers in the United States in the J1772 Standard. Figure 7 summarizes these attributes and the estimated recharge times. Actual recharge times depend on the onboard equipment, including the charger, battery, and battery management system.

Most PEV manufacturers supply an AC Level 1 cord-set with the vehicle, which provides sufficient capabilities for some drivers, but often provides an emergency backup capability because of the long recharge times. AC recharging capabilities found in the public arena more typically are AC Level 2. Figure 8 depicts a typical J1772-compliant inlet and connector for both AC Levels 1 and 2.

The J1772 standard also identifies requirements for DC charging. For PEVs that accept both AC and DC inputs, the Society of Automotive Engineers approved a single connector and inlet design. Figure 9 shows this connector, which is colloquially known as the J1772 "combo connector."

Some BEVs introduced in the United States prior to the approval of the J1772 standard for DC charging employ the CHAdeMO (designed in Japan) standard for connector and inlet design. Figure 10 shows this connector. EVSE units that are either J1772-compliant or CHAdeMO-compliant are both known as DC fast chargers.

The presence of the two separate standards for DC charging presents challenges for vehicle owners to ensure the EVSE accessed provides the appropriate connector for their vehicle inlet. Not all PEV suppliers include DC charging options. BEV suppliers have provided DC inlets where PHEV suppliers have not, because the rapid recharging provides opportunities for expanded vehicle range with minimal operator wait times. PHEV operators can rely on the gasoline drive in the event they deplete the vehicle's battery; at present, no PHEV on the market or near commercialization has DC charging capability (although the Mitsubishi Outlander PHEV is rumored to be offering DC charging capability as an option).

\footnotetext{
${ }^{19}$ http://www.theevproject.com/downloads/documents/Electric\%20Vehicle\%20Charging\%20Infrastructure\%20Deployment\%20
} Guidelines $\% 20$ for $\% 20$ the $\% 20$ Greater\%20Phoenix\%20Area\%20Ver\%203.2.pdf [accessed January 15, 2014]. 
It is noted that DC Level 1 and DC Level 2 charging are commonly combined and labeled "DC fast charging."

\begin{tabular}{|c|c|c|c|}
\hline \multicolumn{4}{|c|}{ SAE Charging Configurations and Ratings Terminology } \\
\hline \multirow{5}{*}{$\begin{array}{l}\text { AC level } 1 \\
\left(\text { SAE } J 1772^{m-1}\right)\end{array}$} & PEV includes on-board charger & \multirow[t]{5}{*}{ *DC Level 1} & EVSE includes an off-board charger \\
\hline & $\begin{array}{l}120 \mathrm{~V}, 1.4 \mathrm{~kW} @ 12 \mathrm{amp} \\
120 \mathrm{~V}, 1.9 \mathrm{~kW} @ 16 \mathrm{amp}\end{array}$ & & $200-450 \mathrm{~V} \mathrm{DC}$, up to $36 \mathrm{~kW}(80 \mathrm{~A})$ \\
\hline & Est. charge time: & & Est. charge time ( $20 \mathrm{~kW}$ off-board charger): \\
\hline & PHEV: 7 hrs (SOC* $-0 \%$ to full) & & PHEV: $22 \mathrm{~min}$. (SOC* $-0 \%$ to $80 \%$ ) \\
\hline & BEV: $17 \mathrm{hrs}$ (SOC $-20 \%$ to full) & & BEV: 1.2 hrs. (SOC $-20 \%$ to $100 \%)$ \\
\hline \multirow[t]{11}{*}{$\begin{array}{l}\text { AC level } 2 \\
\text { (SAE J1772 }\end{array}$} & $\begin{array}{l}\text { PEV includes on-board charger (see below for different } \\
\text { types) }\end{array}$ & \multirow[t]{6}{*}{ *DC Level 2} & EVSE includes an off-board charger \\
\hline & $240 \mathrm{~V}$, up to $19.2 \mathrm{~kW}(80 \mathrm{~A})$ & & $200-450 \mathrm{~V} \mathrm{DC}$, up to $90 \mathrm{~kW}(200 \mathrm{~A})$ \\
\hline & Est. charge time for $3.3 \mathrm{~kW}$ on-board charger & & Est. charge time ( $45 \mathrm{~kW}$ off-board charger): \\
\hline & PEV: 3 hrs (SOC* - $0 \%$ to full) & & PHEV: $10 \mathrm{~min}$. (SOC* $-0 \%$ to $80 \%$ ) \\
\hline & BEV: 7 hrs (SOC $-20 \%$ to full) & & BEV: $20 \mathrm{~min}$. (SOC $-20 \%$ to $80 \%$ ) \\
\hline & Est. charge time for $7 \mathrm{~kW}$ on-board charger & & \\
\hline & PEV: 1.5 hrs (SOC* $-0 \%$ to full) & \multirow[t]{6}{*}{ *DC Level 3 (TBD) } & EVSE includes an off-board charger \\
\hline & BEV: 3.5 hrs (SOC $-20 \%$ to full) & & $200-600 \mathrm{~V} \mathrm{DC}$ (proposed) up to $240 \mathrm{~kW}(400 \mathrm{~A})$ \\
\hline & Est. charge time for $20 \mathrm{~kW}$ on-board charger & & Est. charge time ( $45 \mathrm{~kW}$ off-board charger): \\
\hline & PEV: $22 \mathrm{~min}$. (SOC* $-0 \%$ to full) & & BEV (only): $<10 \mathrm{~min}$. (SOC* $-0 \%$ to $80 \%$ ) \\
\hline & BEV: $1.2 \mathrm{hrs}$ (SOC $-20 \%$ to full) & & \\
\hline -AC Level 3 (TBD) & $>20 \mathrm{~kW}$, single phase and 3 phase & & \\
\hline \multicolumn{4}{|c|}{$\begin{array}{l}\text { "Not finalized } \\
\text { Voltages are nominal configuration voltages, not coupler ratings } \\
\text { Rated Power is at nominal configuration operating voltage and coupler rated current } \\
\text { ldeal charge times assume } 90 \% \text { efficient chargers, } 150 \mathrm{~W} \text { to } 12 \mathrm{~V} \text { loads and no balancing of Traction Battery Pack }\end{array}$} \\
\hline \multicolumn{4}{|r|}{ ne hour) will also stop at $80 \%$ SOC instead of } \\
\hline
\end{tabular}

Figure 7. Society of Automotive Engineers charging configurations and ratings terminology. ${ }^{20}$

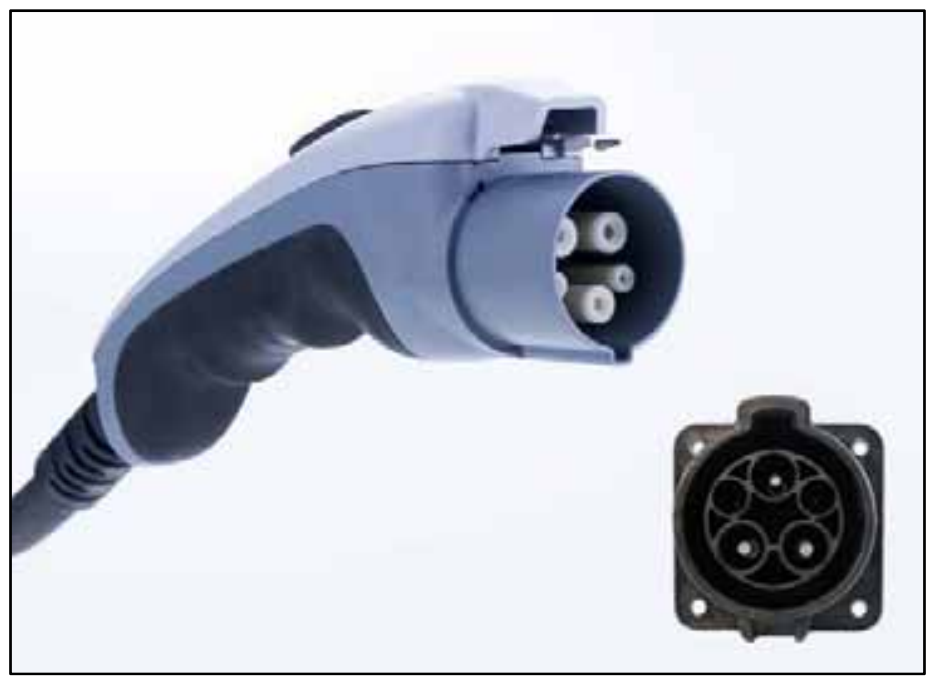

Figure 8. J1772 connector and inlet. ${ }^{21}$

${ }^{20}$ http://www.sae.org/smartgrid/chargingspeeds.pdf [accessed January 15, 2014]. 

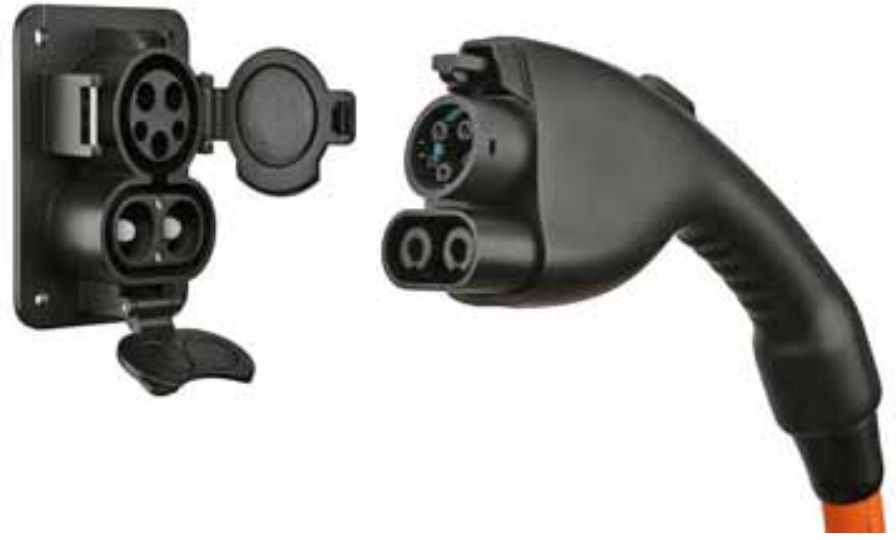

Figure 9. J1772-compliant combo connector ${ }^{22}$.

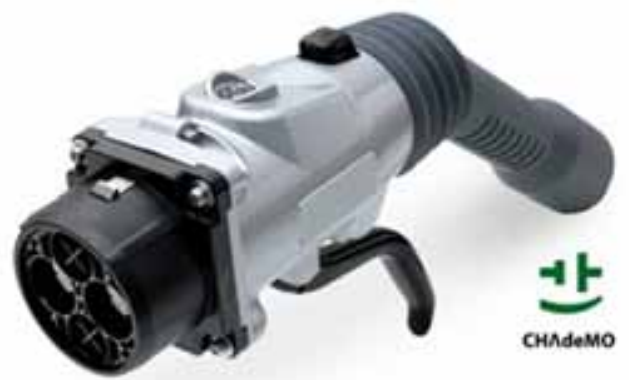

Figure 10. CHAdeMO-compliant connector ${ }^{23}$

Because the battery of a BEV is typically much larger than that of a PHEV, recharge times are longer (see Figure 7). BEVs that see daily mileage near the limits of the advertised range do better when recharged using AC Level 2 EVSE or DC fast charging, because AC Level 1 recharge times are usually extensive. PHEVs, on the other hand, generally can use AC Level 1 EVSE for overnight charging to ensure a fully charged battery at the start of daily use. AC Level 2 EVSE units provide greater range in the shortest amount of time when intermediate or opportunity charging. DC fast charging provides the fastest recharge capability for those vehicles equipped with DC fast charge inlets.

\subsubsection{Electric Vehicle Supply Equipment Stations}

AC Level 2 charging is the predominant rating of publicly accessible EVSE because of its wide acceptance by auto manufacturers and recharge times that are faster than AC Level 1 charging. Purchase and installation costs are more manageable than DC fast chargers and less space is required. There are several manufacturers of AC Level 2 equipment and the agency should review brands for comparison purposes. Figure 10 provides an example of a public AC Level 2 EVSE unit ${ }^{24}$.

\footnotetext{
${ }^{21} \mathrm{http}: / /$ carstations.com/types/j09 [accessed January 15, 2014].

${ }^{22} \mathrm{http}$ ://www.zemotoring.com/news/2012/10/sae-standardizes-j1772-fast-dc-charging-up-to-100-kw [accessed January 15, 2014].

${ }^{23}$ https://radio.azpm.org/p/azspot/2012/5/10/1632-electric-cars/ [accessed January 15, 2014].

${ }^{24}$ www.eaton.com/ [accessed January 29, 2014].
} 


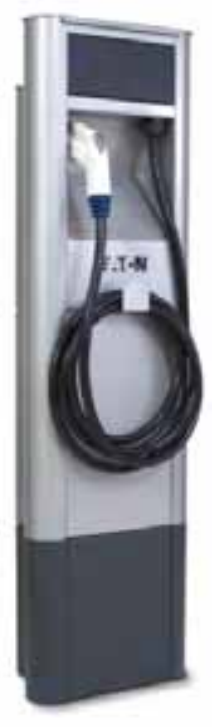

Figure 11. Public alternating current Level 2 electric vehicle support equipment.

DC fast chargers also are available from several manufacturers. Figure 12 illustrates one such charger. ${ }^{25}$ This particular charger uses the CHAdeMO connector standard.

In general, installation costs are higher for the DC fast charger because of the higher voltage requirements and the inclusion of the $\mathrm{AC}$ to $\mathrm{DC}$ converter and other safety and design features. Costs for both types are highly dependent on site characteristics such as distance to the nearest power source, asphalt or concrete cutting and repair, conduit requirements, and payment systems, if any.

Payment and equipment control systems included by some suppliers provide the potential for use by privately owned vehicles for a fee, but can allow agency fleet vehicle use without direct payment. These systems also allow for accurate record keeping of vehicle charging requirements.

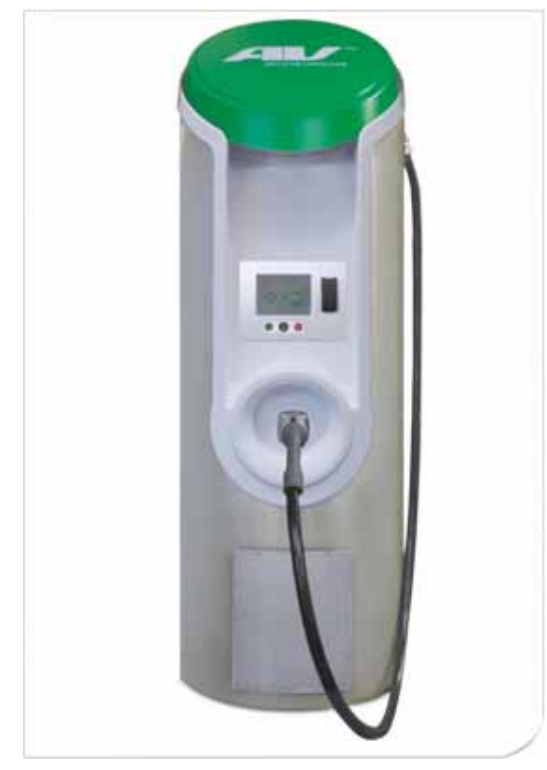

Figure 12. Public direct current fast charger unit.

${ }^{25} \mathrm{http}: / /$ evsolutions.avinc.com/products/public_charging/public_charging_b [Accessed April 16, 2014]. 


\section{GRAND CANYON NATIONAL PARK ANALYSIS \\ 5.1 Grand Canyon National Park Fleet}

GCNP reports a total of 232 interior and GSA vehicles in their complete fleet. Table 8 shows the breakdown of EPA vehicle class by park division.

Table 8. Grand Canyon National Park fleet vehicles.

\begin{tabular}{|c|c|c|c|c|c|c|c|c|c|c|c|}
\hline Division & $\begin{array}{c}\text { Sedan } \\
\text { Compact }\end{array}$ & $\begin{array}{c}\text { Sedan } \\
\text { Midsize }\end{array}$ & $\begin{array}{l}\text { Sedan } \\
\text { Large }\end{array}$ & SUV & $\begin{array}{c}\text { Mini- } \\
\text { van }\end{array}$ & $\begin{array}{c}\text { Cargo } \\
\text { Van } \\
\end{array}$ & $\begin{array}{l}\text { Pass } \\
\text { Van }\end{array}$ & $\begin{array}{c}\text { Pickup } \\
\text { or Light- } \\
\text { Duty } \\
\text { Truck } \\
\end{array}$ & $\begin{array}{c}\text { Heavy- } \\
\text { Duty } \\
\text { Truck }\end{array}$ & Specialty & Total \\
\hline Administration & & 1 & & 6 & 2 & & & & & & 9 \\
\hline Concessions & 2 & & 1 & 2 & & & & & & & 5 \\
\hline Interpretation & 1 & 1 & & 4 & 2 & & & 1 & & & 9 \\
\hline Maintenance & & 1 & 2 & 8 & 1 & 3 & 3 & 80 & 2 & 1 & 101 \\
\hline PMT & & 1 & & 2 & & & & & & & 3 \\
\hline Science/Resource & 1 & & & 7 & & & 1 & 12 & 1 & & 22 \\
\hline Supt Office & & 2 & & 6 & & & & 1 & & & 9 \\
\hline Visitor/Resource & 1 & 1 & 3 & 36 & & & 2 & 15 & & & 58 \\
\hline Fire & & & & 3 & & & & 9 & 1 & 3 & 16 \\
\hline Total & 5 & 7 & 6 & 74 & 5 & 3 & 6 & 118 & 4 & 4 & 232 \\
\hline
\end{tabular}

Based on GCNP input on fleet vehicles, an assessment of mission by vehicle type was completed.

Table 9 shows the results of that assessment.

Table 9. Grand Canyon National Park fleet mission assessment.

\begin{tabular}{|c|c|c|c|c|c|c|c|c|c|c|c|}
\hline Mission & $\begin{array}{c}\text { Sedan } \\
\text { Compact }\end{array}$ & $\begin{array}{c}\text { Sedan } \\
\text { Midsize }\end{array}$ & $\begin{array}{l}\text { Sedan } \\
\text { Large }\end{array}$ & SUV & $\begin{array}{l}\text { Mini- } \\
\text { van }\end{array}$ & $\begin{array}{c}\text { Cargo } \\
\text { Van }\end{array}$ & $\begin{array}{l}\text { Pass } \\
\text { Van }\end{array}$ & $\begin{array}{l}\text { Pickup or } \\
\text { Light-Duty } \\
\text { Truck }\end{array}$ & $\begin{array}{c}\text { Heavy- } \\
\text { Duty } \\
\text { Truck }\end{array}$ & Specialty & Total \\
\hline Pool & 4 & 6 & 3 & 23 & 4 & 3 & 2 & 61 & & & 106 \\
\hline Support & 1 & 1 & 3 & 39 & 1 & & 4 & 56 & 4 & & 109 \\
\hline Law Enforcement & & & & 12 & & & & 1 & & & 13 \\
\hline Specialty & & & & & & & & & & 4 & 16 \\
\hline Total & 5 & 7 & 6 & 74 & 5 & 3 & 6 & 118 & 4 & 4 & 232 \\
\hline
\end{tabular}

\subsection{Survey Results}

Eleven vehicles were included in the study at GCNP. Five vehicles have pool missions and six are support vehicles. Table 10 presents a summary of these vehicles and Table 11 provides details of the monitored vehicles. The Fleet Vehicle IDs are the last five digits of the VIN.

Table 10 . Vehicle study summary.

\begin{tabular}{cccccccc} 
Mission & $\begin{array}{c}\text { Sedan - } \\
\text { Compact }\end{array}$ & $\begin{array}{c}\text { Sedan - } \\
\text { Large }\end{array}$ & Minivan & SUV & $\begin{array}{c}\text { Pickup } \\
\text { Truck }\end{array}$ & $\begin{array}{c}\text { Truck } \\
\text { HD }\end{array}$ & Total \\
\hline Pool & & 1 & 1 & 3 & & & 5 \\
Support Vehicle & 1 & & & 2 & 1 & 2 & 6 \\
Total & 1 & 1 & 1 & 5 & 1 & 2 & 11 \\
\hline
\end{tabular}


Table 11. Detailed Grand Canyon National Park vehicle index.

\begin{tabular}{|c|c|c|c|c|c|}
\hline \multicolumn{6}{|c|}{ Vehicle Index } \\
\hline Logger & Make & Model & Year & Fleet Vehicle Id & Mission \\
\hline 67 & Jeep & Liberty & 2006 & 42612 & Support \\
\hline 68 & Chevy & Uplander & 2008 & 79736 & Pool \\
\hline 69 & Ford & F250 & 2009 & 70762 & Support \\
\hline 70 & Ford & F250 & 2011 & 70202 & Support \\
\hline 71 & Chevy & Impala & 2010 & 38768 & Pool \\
\hline 72 & Ford & Explorer & 2010 & 67912 & Pool \\
\hline 73 & Dodge & Durango & 2008 & 45115 & Pool \\
\hline 74 & Ford & Ranger & 2007 & 90661 & Support \\
\hline 75 & Chevy & HHR & 2010 & 20389 & Pool \\
\hline 79 & Ford & Focus & 2011 & 17523 & Support \\
\hline 80 & Ford & Escape & 2011 & 69685 & Support \\
\hline
\end{tabular}

Specific vehicle references may be made to the vehicle ID or logger ID in this report.

Appendix D provides the analysis of each individual vehicle included in this study. Grouping the vehicles by mission creates an aggregated view of mission requirements to provide observations related to PEV replacement. The missions of these two categories vary considerably; therefore, these missions are evaluated separately, because fleet-wide operations provide little useful information.

\subsection{Data Validity}

GCNP data collection took place from March 29 through July 10, 2013. Vehicle data sheets (presented in Appendix D) detail the collected data for each vehicle, including specific dates the logger provided data.

Of the data collected, validation occurred for $99.3 \%$, while null values exist for the balance. Table 12 shows this information by mission type.

Table 12. Vehicle data logger reporting summary.

\begin{tabular}{lccc}
\hline \multicolumn{4}{c}{ Vehicle Data Logger Reporting Summary } \\
\multicolumn{1}{c}{ Mission } & \% Collected & \% Null Values & Total \\
\hline Pool & 98.5 & 1.5 & $100 \%$ \\
Support Vehicles & 99.4 & 0.6 & $100 \%$ \\
All Vehicles & 99.3 & 0.7 & $100 \%$ \\
\hline
\end{tabular}

\subsection{Pool Vehicles Evaluation}

\subsubsection{Survey and Site Information}

Pool vehicles are typically light-duty motor vehicles for use in passenger transportation, with not more than 10 passengers. Pool missions can vary by agency, location, and jurisdiction; however, they typically utilize sedans, minivans, sport utility vehicles (SUVs), vans, or small pickup trucks and typically do not carry specific cargo or equipment. Table 10 identifies the five vehicles (i.e., three SUVs, a minivan, and a large sedan).

Incorporation of BEVs and/or PHEVs into the pool mission is a definite possibility. Pool vehicles used for shorter trips or outings qualify for BEV or PHEV replacement, while other pool vehicle activities that are associated with longer trips may require PHEV capabilities. 


\subsubsection{Summary for Pool Vehicles}

Appendix D provides the vehicle data sheets for each of the pool vehicles monitored. This section aggregates data for all pool vehicles. Table 13summarizes pool travel during the study period for those days in which the vehicle was driven. Vehicle use occurred primarily between 0700 and 1600 hours daily. The vehicles were driven 6,018 miles, logged 206 hours, and idled for 39 hours during the 103-day study period.

Table 13. Pool vehicles travel summary.

\begin{tabular}{lcccc}
\hline \multicolumn{4}{c}{ Pool Vehicles Travel Summary } & \\
& $\begin{array}{c}\text { Per Day } \\
\text { Average/Peak }\end{array}$ & $\begin{array}{c}\text { Per Outing } \\
\text { Average/Peak }\end{array}$ & $\begin{array}{c}\text { Per Trip } \\
\text { Average/Peak }\end{array}$ & Total \\
\hline Travel Distance (Miles) & $26.9 / 399.9$ & $18.8 / 495.2$ & $4.4 / 198.0$ & 6,018 \\
Travel Time (Minutes) & $55.3 / 451$ & $38.7 / 833.0$ & $9.1 / 221.0$ & 12,396 \\
Idle Time (Minutes) & $10.6 / \mathrm{NA}$ & $7.4 / \mathrm{NA}$ & $1.7 / \mathrm{NA}$ & 2,372 \\
\hline
\end{tabular}

\subsubsection{Pool Vehicles Daily Summary}

Figure 13 identifies daily travel distance and time for all pool vehicles. The green line and bars indicate the typical electric range on a single charge for a PHEV, while the blue line and bars (including the green bars) indicate the same for a BEV. Figures 14 and 15 show the composite history in distance and time traveled for the pool vehicles. In the stacked bar charts of Figures 14 and 15, the contribution of each vehicle is indicated by a different color.

When driven, the average travel distance per day for pool vehicles was 26.9 miles. On $86 \%$ of these vehicle days, the daily travel was less than the 70 miles considered to be within the BEV safe range. That is, while BEV range can vary based on several factors; most BEVs provide at least 70 miles of vehicle range on a single battery charge. Fourteen percent of pool daily travel was greater than 70 miles. Further, $79 \%$ of vehicle travel days were less than 40 miles, which is considered to be within the CD range of a PHEV.

Figures 14 and 15 show that the vehicles were not used every day, although there were many days when most of the vehicles were in use. Vehicles 79736 and 38768 had the highest number of travel days, while Vehicles 20389 and 67912 had the least.

Figure 16 displays the summary of use by time of day for all pool vehicles. Figure 17 shows the outing distances traveled, including data for all pool vehicles.
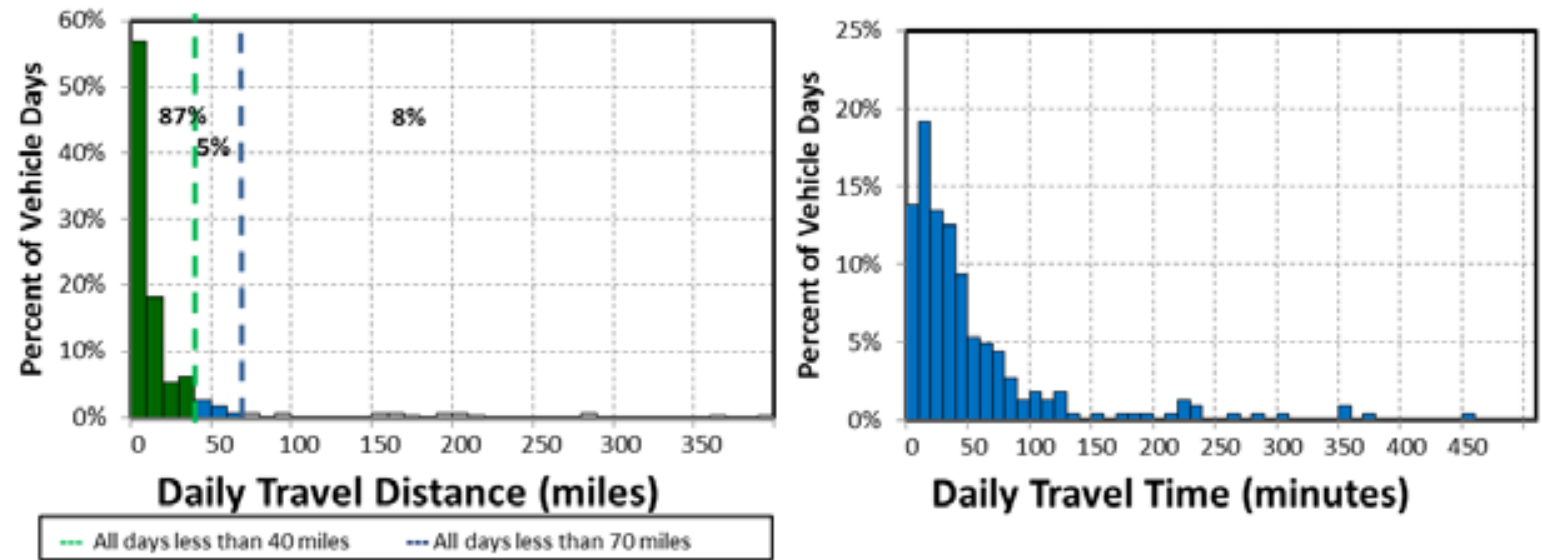

Figure 13. Pool vehicle daily travel miles and time (all vehicles). 


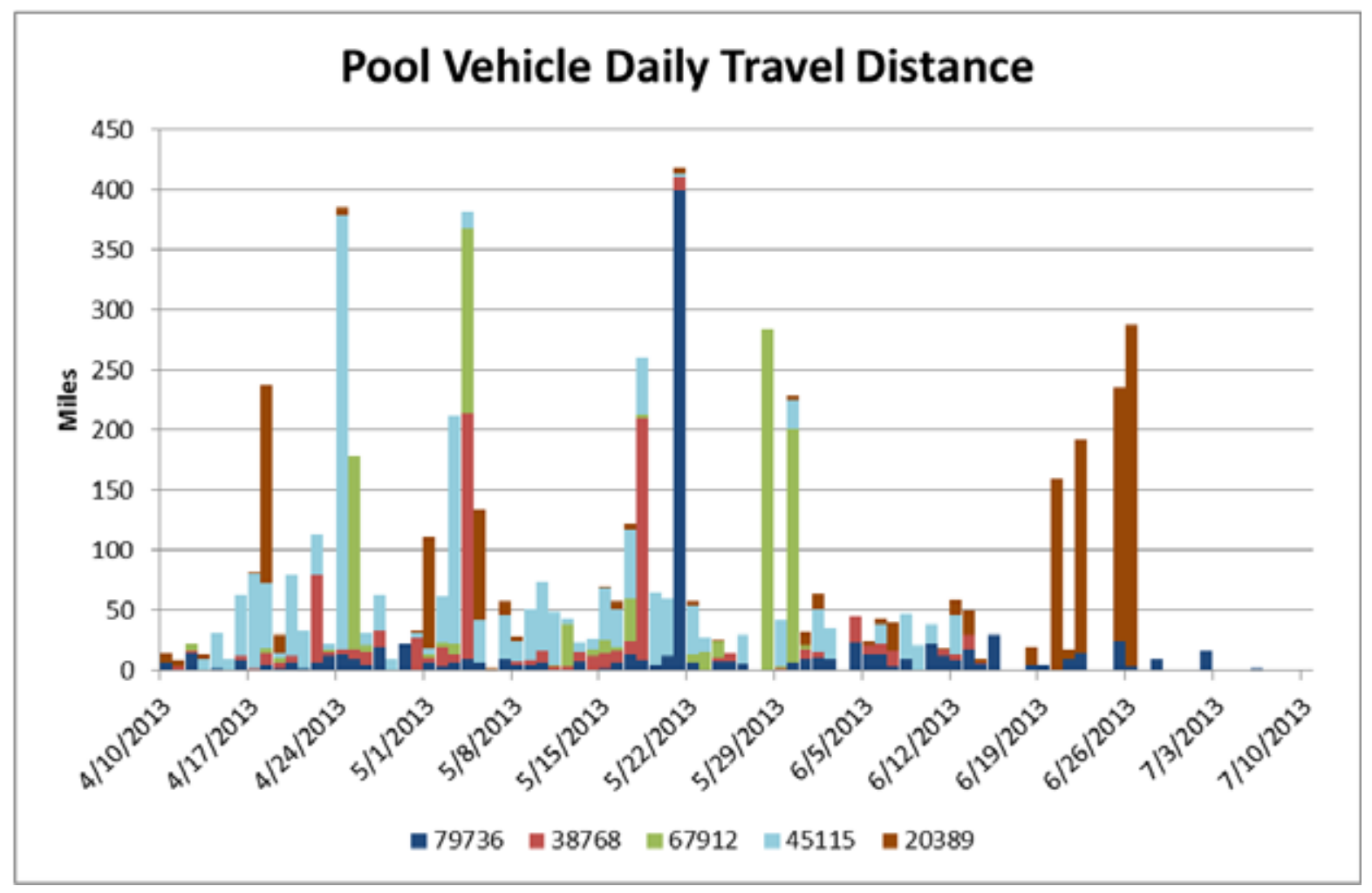

Figure 14. Pool vehicle daily travel history (all vehicles).

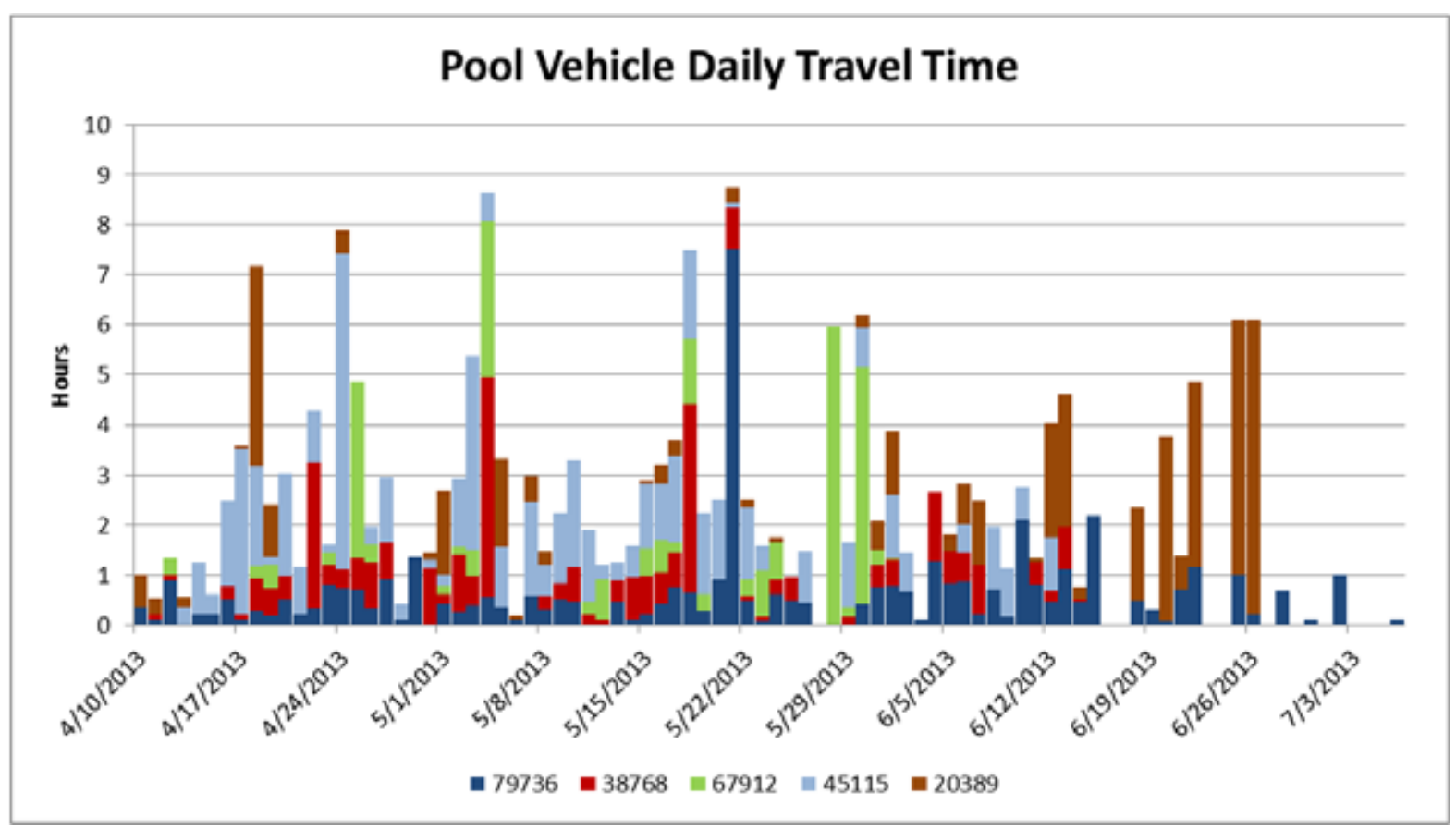

Figure 15. Pool vehicles travel time (all vehicles). 


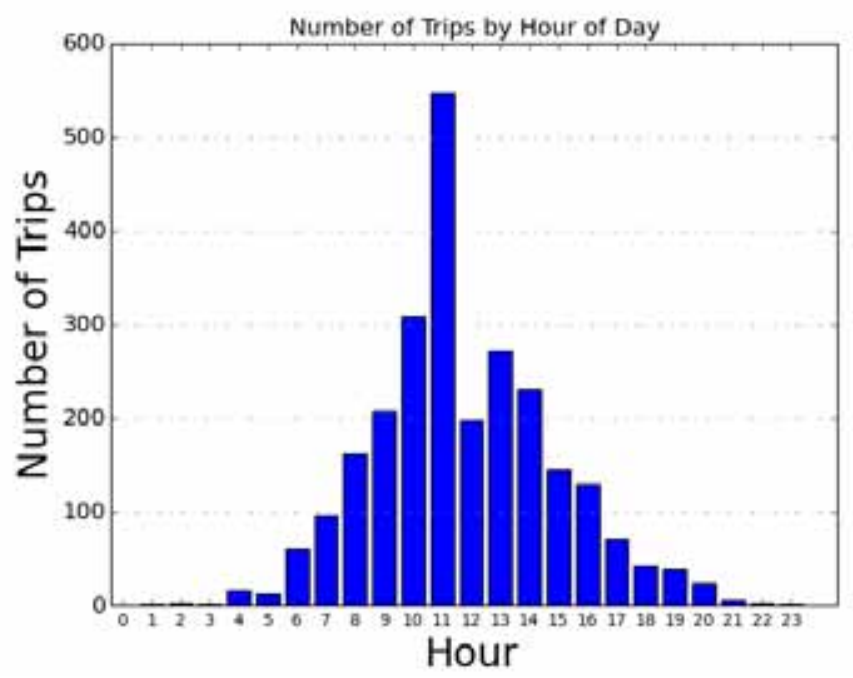

Figure 16. Pool vehicles hourly usage.

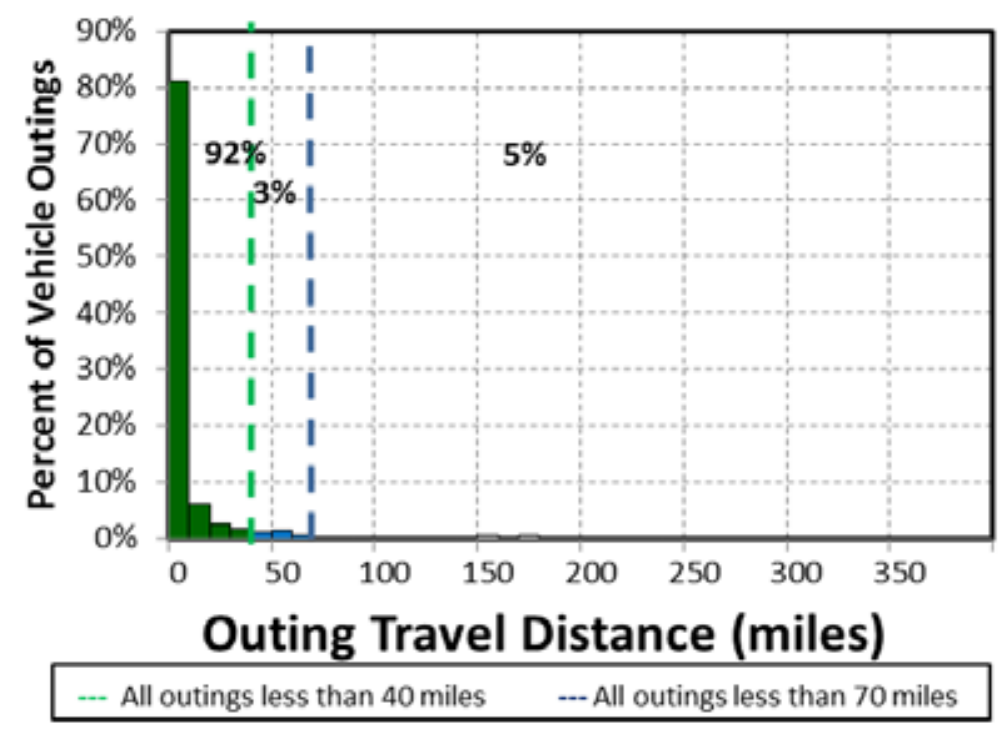

Figure 17. Pool vehicle outings.

Appendix D provides the details of each of the pool vehicle's outing travel. Every vehicle exceeded 70 miles of travel on at least one outing.

The average travel outing for pool vehicles was 18.9 miles. On $95 \%$ of these vehicle outings, the distance traveled was less than the 70 miles considered to be within the BEV safe range. Only $5 \%$ percent of pool outing travel was greater than 70 miles. Further, $92 \%$ of vehicle travel outings were less than the 40 miles considered to be within the CD range of a PHEV. In summary, these vehicles can be characterized by fairly low daily travel and outing distances (the average numbers are quite low), but each vehicle had experienced significantly longer excursions, resulting in high peak daily data.

\subsubsection{Pool Vehicle Observations/Summary}

There appears to be three choices for GCNP in implementing PEVs into the pool fleet. It should be noted that the objective would be to incorporate as many BEVs as possible to realize the advantages of reduced petroleum usage and reduced emissions of GHGs. 
1. All BEV fleet: While some BEV manufacturers report vehicle range exceeding 70 miles, Intertek recommends careful evaluation of experienced range to ensure vehicle missions are accomplished. Nevertheless, assuming the 70-mile safe range for a BEV, an all-BEV fleet does not appear to be possible due to the length of some of the daily travel.

2. Mixed BEV/PHEV fleet: Certainly, PHEVs can accomplish the same mission as the current fleet when only considering travel times and distances, because the PHEV's gasoline engine can provide motive power when the battery has been depleted. Figure 13 shows that on $79 \%$ of all vehicle travel days, the total daily travel is less than 40 miles, which typically is the maximum distance a PHEV will travel in CD mode. This represents a significant operating cost savings opportunity, while retaining the ability to go longer distances when needed. In addition, $92 \%$ of the outings are less than 40 miles and could be completed in CD mode for certain PHEVs if the battery is fully charged prior to the outing.

Meanwhile, $95 \%$ of the outings are within the typical capability of a BEV; therefore, the EVSE at the home base could provide recharge energy for another outing. A mixed fleet requires fleet manager attention to assign vehicles appropriately for the anticipated use on that day.

This would suggest that 5\% of the fleet could be PHEV to handle travel greater than 70 miles per day without requiring additional opportunity charging during daytime stops and $95 \%$ of the fleet could be BEVs. However, this does not allow for the use of several vehicles at the same time and would require a greater level of fleet management, with the daily assignment of vehicles based on anticipated driving distance. Allowing more conservatism in assigning vehicles, three PHEVs and two BEVs could conservatively meet the demand for these five pool vehicles. All monitored pool vehicles were minivans, a sedan, or SUVs and replacement PEVs are currently available for these vehicle types.

3. All PHEV fleet: As noted above, PHEVs can accomplish the same mission as the current fleet when only considering travel times and distances. Replacing all current vehicles with PHEVs only requires an evaluation of the individual vehicle capabilities of currently available PHEVs to meet current pool requirements. These five pool vehicles have replacement PEVs available. Data show that for a significant number of days, the PHEVs will operate in CD mode. The first 40 miles of longer travel days also would be powered by (at least mostly) electricity so that $79 \%$ of all pool vehicle travel would be (again, at least mostly) battery powered with only one charge per day. As above, this represents an opportunity for significant operating cost savings while retaining the ability to go longer distances when needed. Intermediate charging opportunities provide additional benefit, enhancing CD mode. Data show significant charging opportunities throughout the day during stop times.

The vehicle summary shows sufficient time for charging at the base location during the course of the day and additional opportunities at intermediate charging stations are not required. These stations also provide charging opportunities for the visiting public, whose fees may assist in offsetting operating costs. Given the availability of daytime changing, with experience, GCNP may find a greater fraction of BEVs within the pool vehicle fleet may meet their needs.

Considering a full complement of 106 pool vehicles in the total fleet, Intertek notes that two are passenger vans for which no PEV is currently available as a potential replacement. However, for the balance of the vehicles, Intertek suggests that a mixed fleet may be possible. While the remaining vehicles were not monitored, using the same ratio as above suggests that a fleet of 42 BEVs and 62 PHEVs conservatively meets vehicle travel requirements. Typically, additional EVSE at frequently visited locations provides recharging for both the BEV and PHEV that may be of benefit.

The types of vehicles monitored (i.e., minivan, sedan, and SUV) are typical of pool vehicles. Table 9 shows the similarities of the assessed fleet to those vehicles monitored. 


\subsubsection{Pool Vehicle Charging Needs}

Upon review of these data, Intertek suggests replacement of the studied pool fleet with two BEVs and three PHEVs. No available PHEVs at this writing provide for DC fast charging nor do the data suggest that this would be a significant benefit for PHEVs in the pool fleet. A DC fast charger at the home base will provide a more rapid recharge for BEVs, but appears to be unnecessary given that our conservative estimate shows that $95 \%$ of outings are less than a typical BEV's driving range.

As noted above, AC Level 2 overnight charging of BEVs is typical, whereas overnight charging of PHEVs can usually be accomplished with AC Level 1 charging.

Intertek's experience suggests that each vehicle should have an assigned charging parking space at its home base. Assigned stations require less management attention to ensure completion of overnight charging. BEVs and PHEVs not assigned to these stations also benefit during visits to the location as part of their normal operation. For the entire fleet of pool vehicles, the $42 \mathrm{BEVs}$ require $42 \mathrm{AC}$ Level $2 \mathrm{EVSE}$ units for overnight charging and the 64 PHEVs require $64 \mathrm{AC}$ Level 1 outlets at each vehicles overnight parking location. Intertek recommends a minimum of two EVSE at each location to maximize charge capability without a significant increase in installation costs. The PHEVs can utilize the AC Level 2 EVSE at the home base during the day to increase the amount of vehicle miles traveled in CD mode. For these monitored vehicles, Intertek suggests BEVs could replace Vehicles 79736 and 67912. PHEVs could replace Vehicles 38768, 45115, and 20389.

At times, fleet vehicles obtain benefits from using public charging infrastructure. Figure 18 displays the availability of public charging at the time of this writing for the GCNP area. Unfortunately, all EVSE are $\mathrm{AC}$ level 1 (except at the Nissan dealership in Flagstaff) and require extensive recharge time.

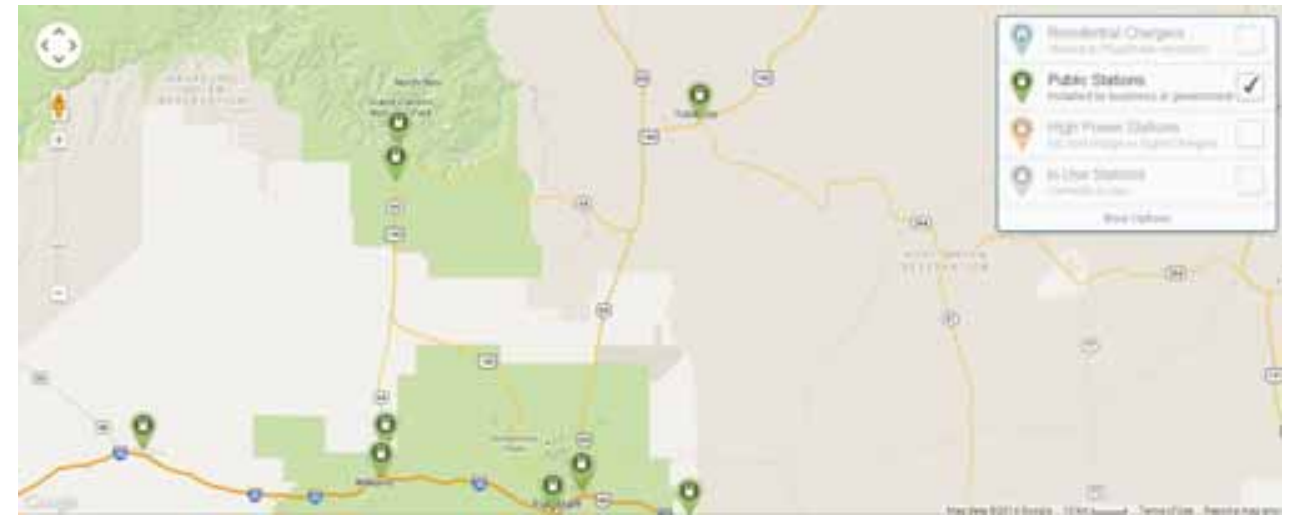

Figure 18. Public electric vehicle supply equipment in the Grand Canyon National Park region ${ }^{26}$.

\subsection{Support Vehicles Evaluation}

Support vehicles provide a specific work function, facilitating the mission of a particular group. The vehicles are generally passenger or light-duty pickup trucks and may contain after-market modifications to support the mission. While assigned to maintenance and service areas, missions may vary depending on agency needs.

\subsubsection{Summary for Support Vehicles}

Appendix D provides the vehicle data sheets for each of the six support vehicles monitored. This section aggregates the data for all support vehicles.

${ }^{26}$ http://www.plugshare.com/ [accessed June 7, 2014]. 
Table 14 summarizes support vehicle travel during the study period. Vehicle use occurred primarily between 0600 and 1400 hours daily. Support vehicles traveled 11,791 miles, logged 500 hours, and idled for 147 hours during the study period.

Table 14. Support vehicle travel summary.

\begin{tabular}{lcccc}
\hline \multicolumn{4}{c}{ Support Vehicle Travel Summary } & \\
& Per Day & Per Outing & Per Trip & \\
& Average/Peak & Average/Peak & Average/Peak & Total \\
\hline Travel Distance (Miles) & $48.2 / 677.1$ & $21.1 / 1,919.7$ & $5.9 / 220.6$ & 11,791 \\
Travel Time (Minutes) & $122.9 / 706.0$ & $53.7 / 3,151.0$ & $15.1 / 290.0$ & 29,986 \\
Idle Time (Minutes) & $36.2 / \mathrm{NA}$ & $15.8 / \mathrm{NA}$ & $4.4 / \mathrm{NA}$ & 8,847 \\
\hline
\end{tabular}

\subsubsection{Support Vehicle Daily Summary}

Figure 19 identifies daily travel distance and time for all support vehicles. The green line and bars indicate typical electric range on a single charge for a PHEV, while the blue line and bars indicate the same for a BEV. Figures 20 and 21 show the composite history in distance and time traveled for the support vehicles. In the stacked bar charts of Figures 20 and 21, the contribution of each vehicle is indicated by a different color.

The history graphs identify when several support vehicles may be in use at the same time and the total miles driven.

During the April to June study period, the average travel distance per day (when driven) for support vehicles was 48.2 miles. On $81 \%$ of these vehicle days, the daily travel was less than the 70 miles considered to be within the BEV safe range. Nineteen percent of support vehicle daily travel was greater than 70 miles, and $68 \%$ of vehicle travel days were less than the 40 miles considered to be within the CD mode of a PHEV.

Figures 19, 20, and 21 show that the vehicles are not used every day, although there are many days when most of the vehicles are in use. Vehicles 70762 and 42612 had the highest number of travel days, while Vehicles 17523 and 69685 had the least. Figure 22 displays the summary of use by time of day for all support vehicles combined. Figure 23 shows the outing distances for all support vehicles. Note that three of the outings at 1,919.7 miles, 1,050.7 miles, and 882.2 miles are not shown for clarity of the remaining data.

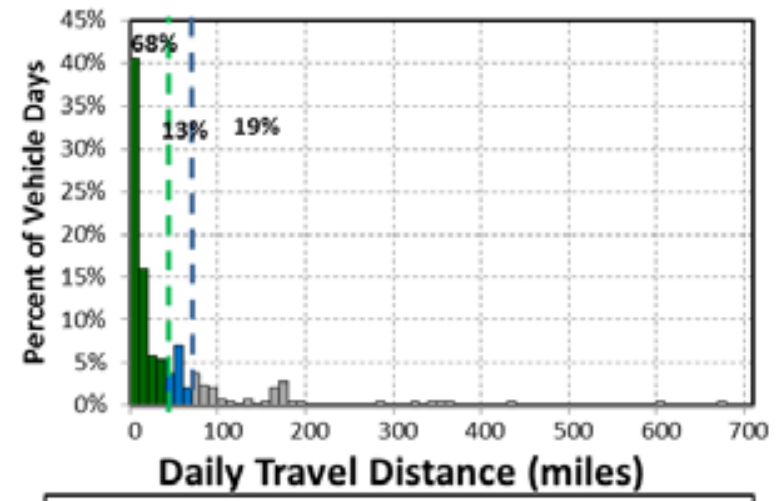

- All days less than 40 miles $\quad$...-All days less than 70 miles

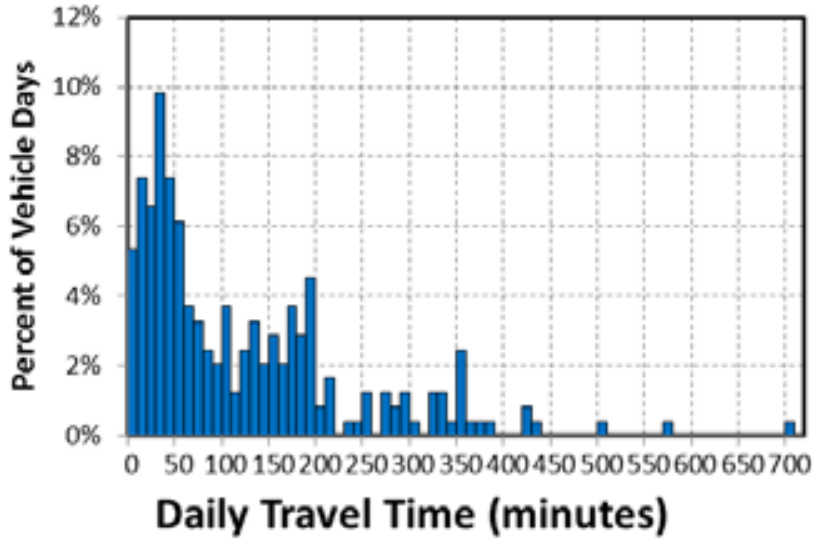

Daily Travel Time (minutes)

Figure 19. Support vehicles percentage of daily use versus daily travel miles and time (all vehicles). 


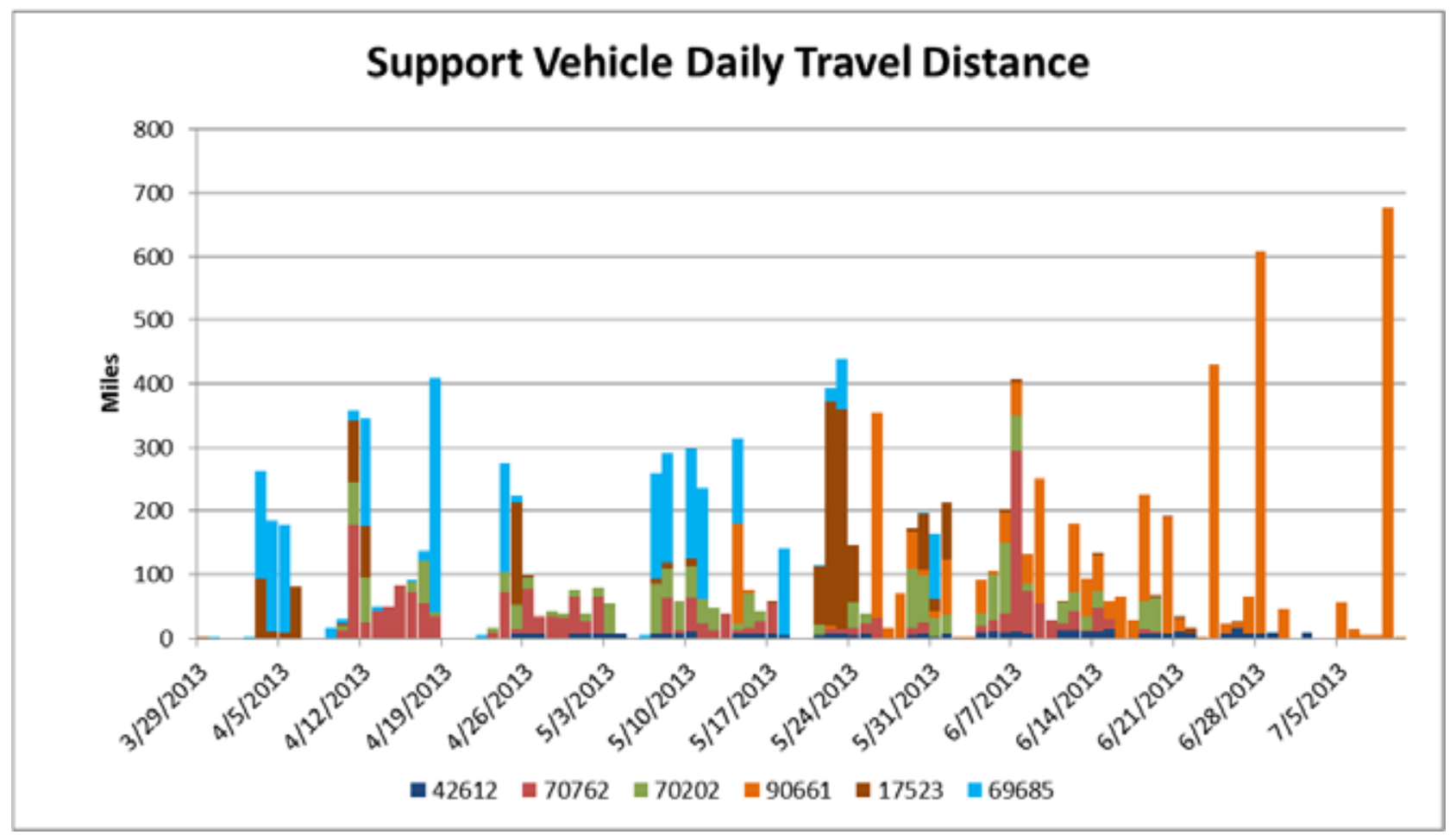

Figure 20. Support vehicle daily travel miles (all vehicles).

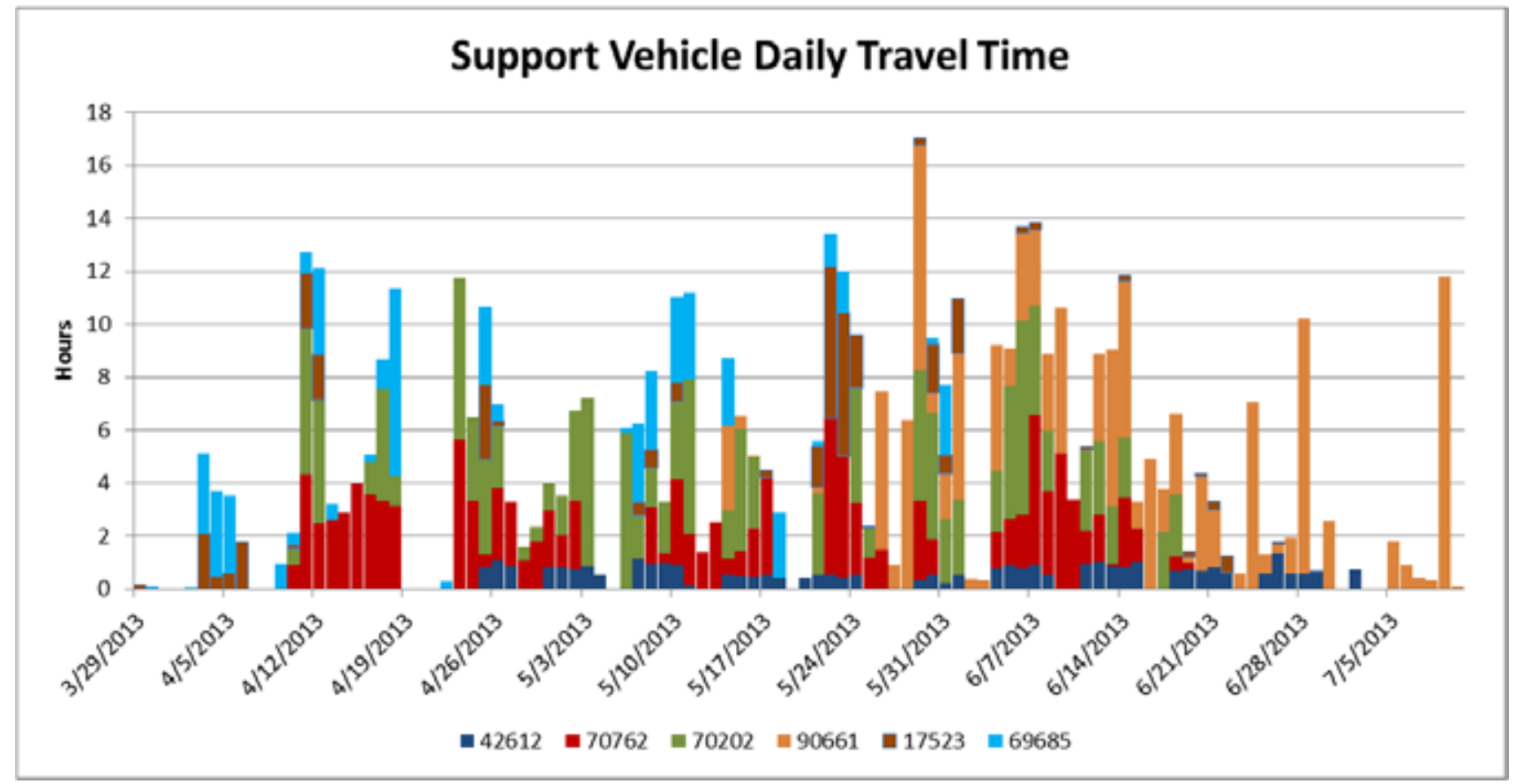

Figure 21. Support vehicle daily travel time (all vehicles). 


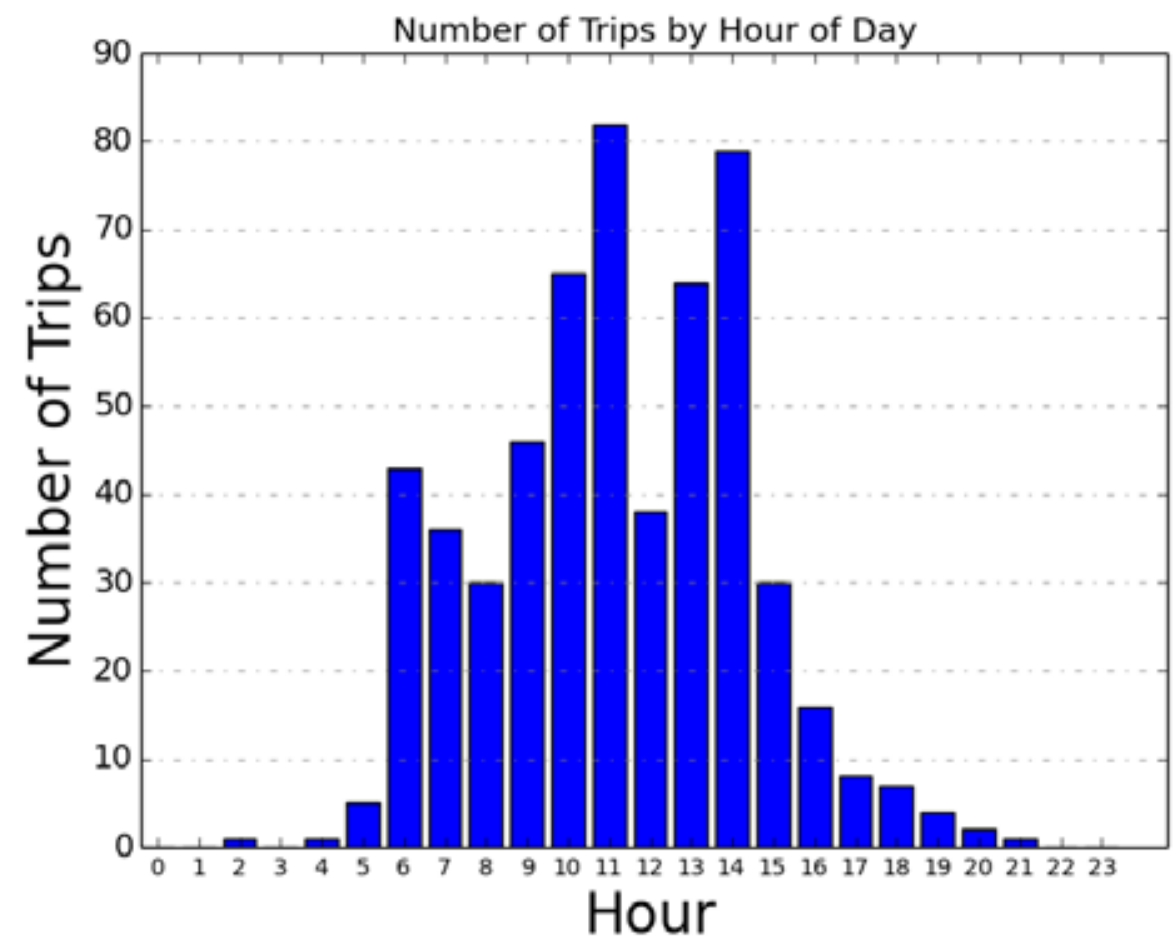

Figure 22. Support vehicles hourly usage.

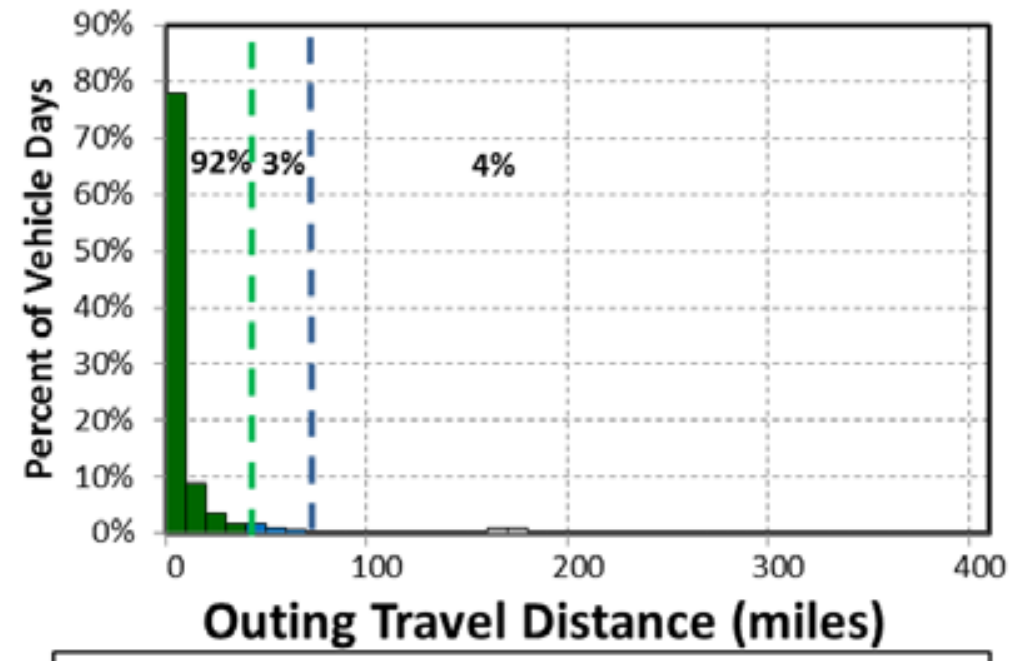

-.. All outings less than 40 miles _..- All outings less than 70 miles

Figure 23. Support vehicle outings.

Appendix D provides the details of each of the support vehicle's daily travel. Vehicle 42612 was the only vehicle that did not exceed the 70 miles of daily travel during the study period.

The average travel outing for support vehicles was 21.1 miles. Ninety-two percent of vehicle travel outings were less than 40 miles and are considered to be within the CD mode of a PHEV.

In summary, these vehicles could be characterized by fairly low daily travel and outing distances (the average numbers are quite low), but each experienced significantly longer excursions, which resulted in high peak daily data. 


\subsubsection{Support Vehicle Observations/Summary}

As a group, the support vehicles had infrequent daily travel distances exceeding 70 miles.

The support vehicles studied consisted of one sedan, two SUVs, and three pickup trucks. Pickup trucks are a popular choice for support vehicles because they are versatile to support various types of support activities needed (e.g., special cargo or equipment transport). In some cases, SUVs or minivans can perform the same mission. Section 4.4 provides information on PEV trucks and vans currently or soon to be available.

As before, there appear to be three possible options for RMNPGCNP in implementing PEVs into the support vehicle fleet. It should again be noted that the objective would be to incorporate as many BEVs as possible to realize the advantages of reduced petroleum usage and reduced emissions of GHGs.

1. All BEV fleet: While some BEV manufacturers report vehicle ranges exceeding 70 miles, Intertek recommends careful evaluation of experienced range to ensure vehicle missions are accomplished. Nevertheless, assuming the 70-mile safe range for a BEV, an all-BEV fleet is not possible for support vehicles due to the long distances experienced by the vehicles and the heavy-duty requirements of the vehicles.

2. Mixed BEV/PHEV fleet: Certainly, PHEVs can accomplish the same mission as the current fleet when only considering travel times and distances because the PEV's gasoline engine can provide motive power when the battery has been depleted. Figure 19 shows that on $68 \%$ of all vehicle travel days, the total daily travel was less than 40 miles, which typically is the maximum distance a PHEV will travel in CD mode. This represents a significant operating cost savings opportunity, while retaining the ability to go longer distances when needed. In addition, $92 \%$ of the outings are less than 40 miles and could be completed in CD mode for certain PHEVs if the battery is fully charged prior to the outing.

Fully $95 \%$ of the outings are within the typical capability of a BEV; therefore, EVSE at the home base could provide recharge energy for another outing. A mixed fleet requires fleet manager attention to appropriately assign vehicles for the anticipated use on that day.

The data suggests that $19 \%$ of the fleet could be PHEVs for handling the travel greater than 70 miles per day without requiring additional opportunity charging during daytime stops and $81 \%$ of the fleet could be BEVs. However, this does not allow for the use of several vehicles at the same time and would require a greater level of fleet management, with the daily assignment of vehicles based on anticipated driving distance. Replacing these vehicles with four PHEVs and two BEVs could conservatively meet the demand.

3. All PHEV fleet: As noted above, PHEVs can accomplish the same mission as the current fleet when only considering travel times and distances. Replacing all current vehicles with PHEVs only requires an evaluation of the individual vehicle capabilities of currently available PHEVs to meet current support vehicle requirements.

The vehicle summary shows sufficient time for charging at the base location during the course of the day. These stations also provide charging opportunities for the visiting public, whose fees may assist in offsetting operating costs.

The current fleet contains 109 total support vehicles (whose assessment of mission by vehicle type is shown in Table 9). Intertek suggests further mission evaluation be given to support vehicles when considering the adoption of BEVs and PHEVs. It may be possible to replace some of the heavy-duty pickups with the Via Motors PHEV pickup. However, to be conservative, it is assumed some of the fleet must remain heavy-duty ICE trucks. In addition, the support fleet contains passenger and cargo vans. There are potential PEV replacements for the cargo van, but no replacement currently exists for the passenger vans. Thus, it is assumed that the composition would include approximately four heavy-duty 
pickup trucks and four passenger vans. Of the balance of 101 vehicles, Intertek suggests that $34 \mathrm{BEVs}$ and 67 PHEVs could replace the fleet and continue to carry out the same mission.

\subsubsection{Support Vehicle Charging Needs}

Upon review of these data, Intertek suggests replacement of most of the support vehicle fleet with 34 BEVs and 67 PHEVs. No available PHEVs at the time of this writing provide for DC fast charging nor do the data suggest that this would be a significant benefit for PHEVs in the support vehicle fleet. A DC fast charger at the home base will provide a more rapid recharge for BEVs, but it appears to be unnecessary. The majority of the support vehicle activity occurs during daytime hours, which leaves significant time during the nighttime hours for recharging.

As noted above, AC Level 2 overnight charging of BEVs is typical, whereas overnight charging of PHEVs usually can be accomplished with AC Level 1 charging. Opportunity charging at intermediate stops obtains the greater benefits from AC Level 2 EVSE. However, remote intermediate stop locations were not identified in the data.

For the entire fleet of support vehicles, 34 BEVs require 34 AC Level 2 EVSE for overnight charging and 67 PHEVs require $67 \mathrm{AC}$ Level 1 outlets for home base charging. Intertek recommends a minimum of two EVSE at each location to maximize charge capability without a significant increase in installation costs. As noted above, there are no publicly accessible EVSE in the vicinity to provide significant backup charging resources.

Greater management attention provides the possibility of reducing the overall number of AC Level 2 EVSE. A ratio of two AC Level 2 charging stations to three vehicles typically sustains a normal fleet operation. Fleet managers rotate vehicles on the charger to complete charging of all vehicles in the allotted time. This analysis does assume a fully recharged battery at the start of each day. GCNP will gain experience in this management as the PEV fleet grows.

\subsection{Balance of Fleet Vehicles}

The balance of the GCNP fleet consists of specialty and law enforcement vehicles. Certain select PEVs are being demonstrated for various specialty applications, but none are listed in the GSA schedule. The SUVs and pickup truck utilized by GCNP are typical law enforcement vehicles for which PEVs are available for suitable replacement. Some enforcement functions involve routine travel paths or duties for which BEVs may provide a suitable alternative. However, in all cases, PHEVs can be utilized to replace the enforcement vehicles.

\section{GREENHOUSE GAS EMISSIONS AVOIDED AND FUEL COST REDUCTION ANALYSIS}

PEV substitution for an existing conventional vehicle avoids GHG emissions and reduces fuel costs. The GHG emissions avoided occur due to the difference in emissions associated with power plant electricity generation versus fuel combustion that occurs in the engine of a conventional vehicle. This analysis does not account for life-cycle emissions that occur outside of electricity generation and fuel combustion phases (i.e., materials and resource extraction, production supply chains, and decommissioning are not accounted for). These phases are beyond the scope of this report due to the significant effort required to conduct an accurate environmental life-cycle assessment for a transportation system in a very specific setting. The analysis used is known as a "tank-to-wheel" analysis rather than a "well-to-wheel" analysis that would include the aforementioned phases. Cost reduction also occurs because the cost of electricity is comparable to the cost of gasoline on a unit of energy basis; however, PEVs are more efficient than conventional ICE vehicles. Because fuel logs were not kept, the mileage accumulated by each vehicle and the extrapolation to annual miles provide the source of fuel consumption estimates for the study vehicles. 
In order to perform the analysis, EPA fuel economy ratings are used. ${ }^{27}$ Tables 15 and 16 provide these ratings. Ratings for the PHEVs in Table 16 include CD operation. Because these data are estimates, assumptions include the following:

1. PHEVs operate in CD mode only for the percentage of travel less than 40 miles per day. This is reasonable for most daily operations, as described in Section 5. This is conservative because additional charge time exists between most outings. It is also conservative because the replacement PEV typically will have greater fuel economy when operating in CS mode. BEVs operate in electric mode for $100 \%$ of travel.

2. Energy consumption for the Mitsubishi Outlander is assigned the same value as the RAV4 EV and Via Motors VTRUX PU and is estimated because EPA has not yet created ratings for these vehicles.

3. Table 16 suggests the PEVs for replacing the existing fleet vehicles, with the exception of the heavy-duty pickups and passenger vans. See Section 4.4 for vehicle availability. For the entire fleet of support vehicles (where a mix of PHEVs and BEVs are possible) the Toyota RAV4 EV or Nissan LEAF is suggested for BEVs ${ }^{28}$.

4. Annual miles are calculated from the actual miles identified in the study and extrapolated to a full 365-day year. This is compared to the annual miles reported by GCNP for information. The GCNP annual miles are used for reduction calculations. Miles in CD mode are the GCNP annual miles times percent of daily travel less than 40 miles for the PHEV replacement and full annual miles for the BEV replacement.

Table 17 provides a pictorial view of potential replacement PEVs.

Table 15. U.S. Environmental Protection Agency fuel economy ratings for current fleet vehicles.

\begin{tabular}{cccccc}
\hline Vehicle & Logger & Mission & Make \& Model & Model Year & $\begin{array}{c}\text { Fuel Economy-Combined } \\
\text { (miles/gallon) }\end{array}$ \\
\hline 42612 & 67 & Support & Jeep Liberty & 2006 & 17 \\
79736 & 68 & Pool & Chevrolet Uplander & 2008 & 19 \\
70762 & 69 & Support & Ford F250 & 2009 & 16 \\
70202 & 70 & Support & Ford F250 & 2011 & 14 \\
38768 & 71 & Pool & Chevrolet Impala & 2010 & 17 \\
67912 & 72 & Pool & Ford Explorer & 2010 & 16 \\
45115 & 73 & Pool & Dodge Durango & 2008 & 15 \\
90661 & 74 & Support & Ford Ranger & 2007 & 23 \\
20389 & 75 & Pool & Chevrolet HHR & 2010 & 26 \\
17523 & 79 & Support & Ford Focus & 2011 & 31 \\
69685 & 67 & Support & Ford Escape & 2011 & 20 \\
\hline
\end{tabular}

\footnotetext{
${ }^{27}$ http://www.fueleconomy.gov/feg/Find.do?action=sbs\&id=33558 [accessed February 2, 2014].

${ }^{28}$ An SUV BEV replaces the pickups due to current market availability. PEV pickup trucks will be increasingly available in the near future. One example is the PHEV Via Motors VTRUX (http://www.viamotors.com/wp-content/uploads/VIA-SmallBrochure.pdf). [accessed July 19, 2013].
} 
Table 16. U.S. Environmental Protection Agency plug-in electric vehicle energy consumption assumptions.

\begin{tabular}{ccc}
\hline Mission & Replacement PEV & Wh/mile \\
\hline Support & Nissan Leaf & 300 \\
Pool & Nissan Leaf & 300 \\
Support & Via Motors VTRUX & 475 \\
Support & Toyota Rav4 & 440 \\
Pool & Ford Fusion & 370 \\
Pool & Toyota Rav4 & 440 \\
Pool & Mitsubishi Outlander & 440 \\
Support & Via Motors VTRUX & 475 \\
Pool & Mitsubishi Outlander & 440 \\
Support & Chevrolet Volt & 350 \\
Support & Mitsubishi Outlander & 440 \\
\hline
\end{tabular}

Table 17. Plug-in electric vehicle substitutions for current vehicles.

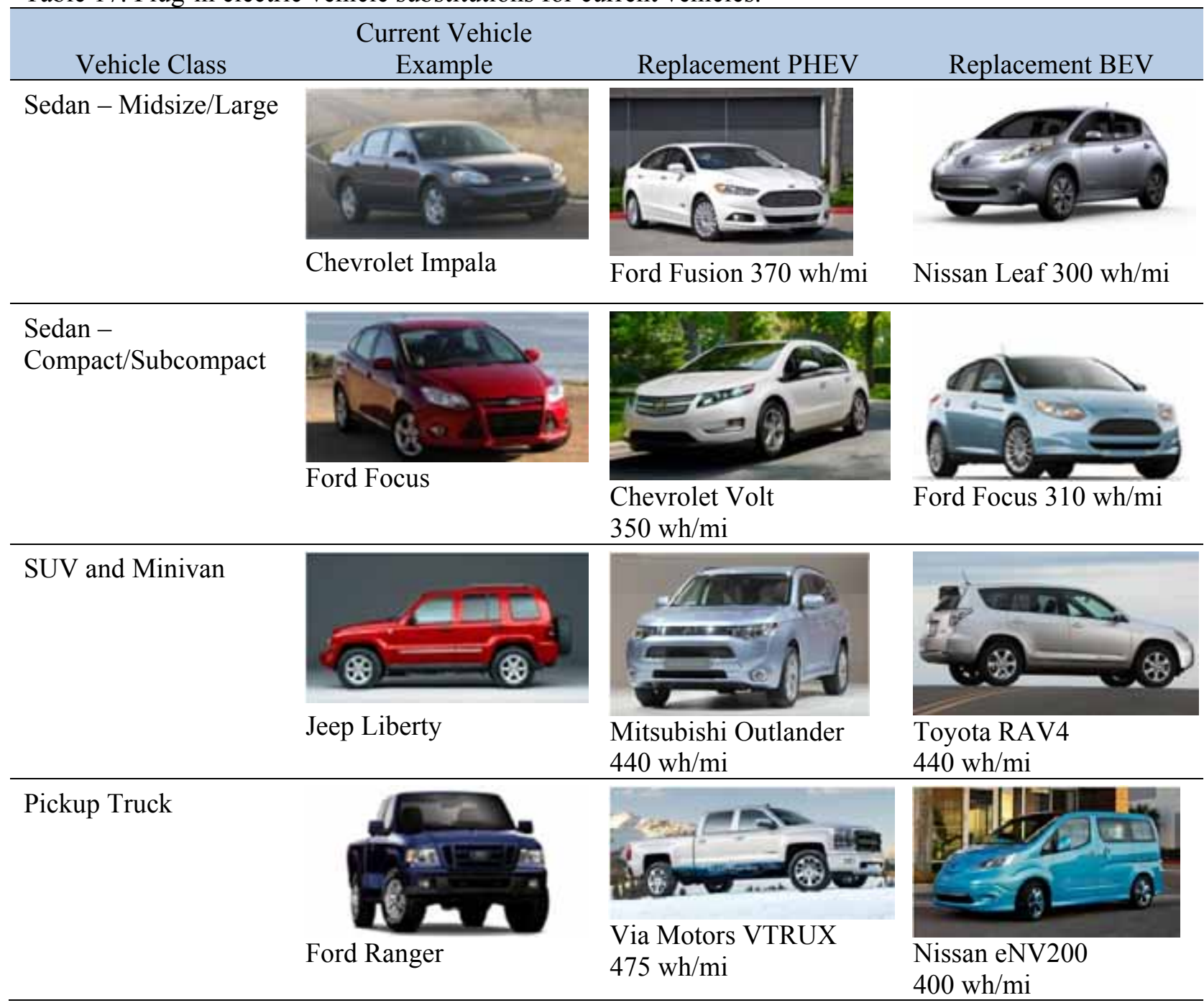




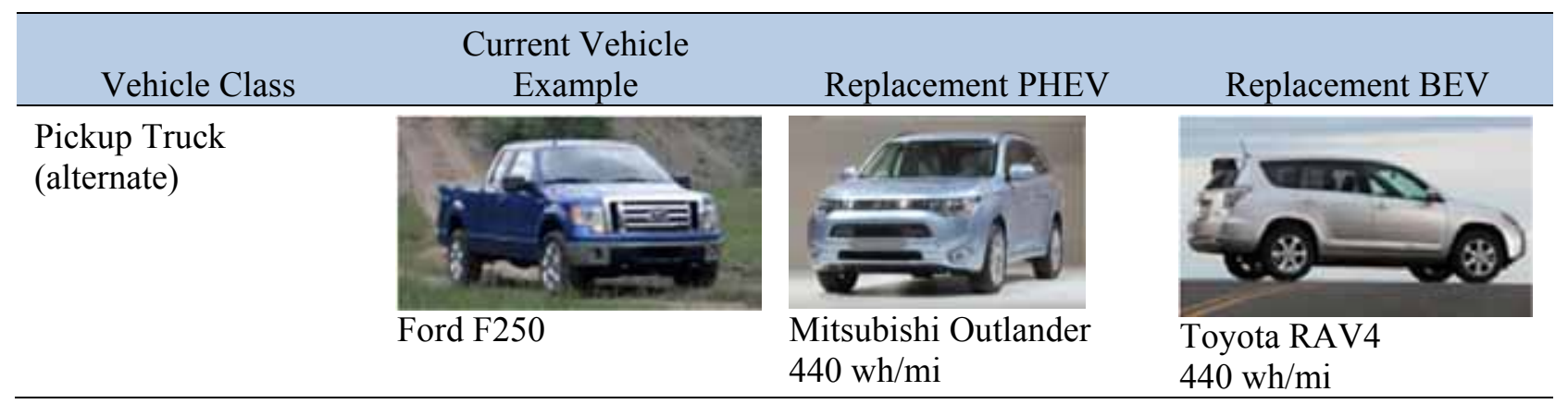

Calculations provided for GHG emissions and fuel savings include both a total United States perspective and local area perspective. The electricity generation mix of power plants for the total United States is different from the local mix of generation in the GCNP area. Likewise, the national average cost for petroleum fuel is different from the local cost for fuel. This analysis includes both approaches in order to allow for local evaluation and to provide the potential benefit for fleet vehicles in other locations of the United States that may be of interest. The final report from Intertek to Idaho National Laboratory summarizing results from all sites studied across the United States primarily will consider the national figures.

For the GHG emissions avoided portion of the analysis, the GHG emissions (in pounds of carbon dioxide equivalent (which also accounts for other GHGs such as methane and nitrous oxide), $l b-\mathrm{CO}_{2} e$, from combustion of gasoline is $20.1 \mathrm{lb}-\mathrm{CO}_{2} \mathrm{e} /$ gallon. ${ }^{29}$ The United States averages for GHG emissions for the production of electricity is $1.53 \mathrm{lb}-\mathrm{CO}_{2} \mathrm{e} / \mathrm{kWh}^{30}$.

GCNP electric power is provided by Arizona Public Service. Arizona Public Service reports a mix of generation from several power plants, including nuclear, coal, gas, solar, and wind generation ${ }^{31}$. EPA reports GHG emissions from the production of electricity. The annual report is available in the Emissions and Generation Resource Integrated Database. The most recent publication is for $2010^{32}$. Using the generation mix reported by Arizona Public Service and the Emissions and Generation Resource Integrated Database plant reports, emissions for 2010 for the production of electricity were $0.874 \mathrm{lb}-\mathrm{CO}_{2} \mathrm{e} / \mathrm{kWh}$. This emission rate reflects high local dependence on nuclear as generation fuel.

GHG emissions avoided are the GHG emissions emitted by the current vehicle (total annual gallons gasoline $\times$ GHG emissions/gallon) minus the annual GHG emitted by the replacement PEV (total annual $\mathrm{kWh} \times \mathrm{GHG}$ emissions $/ \mathrm{kWh}$ ). For PHEVs, the percentages of outings less than 40 miles are counted for the annual miles saved in CD mode, with the balance of the miles accounted as fueled with gasoline.

Table 18 shows the calculation of annual miles based on the recorded and extrapolated miles in this study. GCNP's reported annual miles are also shown for comparison. A replacement vehicle is identified for each vehicle. It is important to note that the analysis conducted suggests replacement vehicles for the fleet of vehicles rather than necessarily replacing the exact vehicle monitored. The percent of miles in CD mode is $100 \%$ for BEVs because all travel is battery powered. The percent of miles in CD mode for PHEVs is obtained from the daily travel shown in Appendix D. Therefore, miles in CD mode is the percentage of GCNP reported annual miles.

\footnotetext{
${ }^{29} \mathrm{http}: / / w w w . t h e e v p r o j e c t . c o m / c m s-a s s e t s / d o c u m e n t s / 106077-891082 . g h g . p d f$ [accessed 19 July 2013].

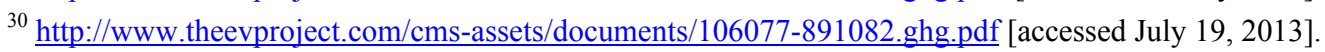

${ }^{31} \mathrm{http}: / /$ www.aps.com/en/ourcompany/news/latestnews/Pages/new-report-shows-changing-arizona-energy-mix.aspx [accessed July 30. 2014].

32 http://www.epa.gov/cleanenergy/energy-resources/egrid/ [accessed June 8, 2014].
} 
Table 18. Miles calculations for charge-depleting mode.

\begin{tabular}{cccccc}
\hline Vehicle & $\begin{array}{c}\text { Replacement } \\
\text { Vehicle }\end{array}$ & $\begin{array}{c}\text { Study Calculated } \\
\text { Annual Miles }\end{array}$ & $\begin{array}{c}\text { GCNP } \\
\text { Reported } \\
\text { Annual Miles }\end{array}$ & $\begin{array}{c}\text { Percent of } \\
\text { Miles CD } \\
\text { Mode }\end{array}$ & $\begin{array}{c}\text { CD Mode } \\
\text { Miles }\end{array}$ \\
\hline 42612 & Leaf & 1,904 & 3,566 & $100 \%$ & 3,566 \\
79736 & Leaf & 4,112 & 11,429 & $100 \%$ & 11,429 \\
70762 & VTRUX PU & 9,954 & 10,131 & $69 \%$ & 6,991 \\
70202 & Rav4 & 8,226 & 15,628 & $100 \%$ & 15,628 \\
38768 & Fusion & 4,497 & 7,000 & $93 \%$ & 6,510 \\
67912 & Rav4 & 5,890 & 10,000 & $100 \%$ & 10,000 \\
45115 & Outlander & 11,588 & 13,750 & $69 \%$ & 9,488 \\
90661 & VTRUX PU & 24,234 & 5,464 & $48 \%$ & 2,623 \\
20389 & Outlander & 6,525 & 4,994 & $80 \%$ & 3,995 \\
17523 & Volt & 6,944 & 7,000 & $56 \%$ & 3,920 \\
69685 & Outlander & 15,295 & 7,000 & $50 \%$ & 3,500 \\
\hline
\end{tabular}

For the cost-avoided piece of the analysis, fuel cost assumptions are \$3.621/gallon of gasoline for the United States and \$3.530/gallon in Arizona ${ }^{33}$. Electrical costs are $0.0984 \$ / \mathrm{kWh}$ for the United States and $0.0981 \$ / \mathrm{kWh}$ in Arizona ${ }^{34}$. Therefore, fuel costs savings are the current vehicle's calculated annual gasoline cost (total annual gallons gasoline $\times$ cost/gallon) minus the electricity cost (total annual $\mathrm{kWh} \times$ cost $/ \mathrm{kWh}$ ) of the replacement PEV traveling the same distance.

The miles calculated above for CD mode yields estimates for yearly GHG emissions avoided and fuel cost reductions. The results of this analysis (shown in Table 19) demonstrate that the substitution of a conventional ICE vehicle with a PEV can reduce the GHG emissions and fuel costs dramatically. The table also shows the percentage reduction in GHG emissions and fuel costs for ease of comparison. For example, if the Ford Fusion replaces Vehicle 38768, a 73\% reduction in GHG emissions in Arizona occurs. The Impala produces 7,697 lb- $\mathrm{CO}_{2 \mathrm{e}}$ year for the distance traveled, whereas the Fusion produces $2,105 \mathrm{lb}-\mathrm{CO}_{2 \mathrm{e}} /$ year for that same distance for a reduction of 5,592 $\mathrm{lb}-\mathrm{CO}_{2 \mathrm{e}} /$ year.

Table 19. Greenhouse gas emissions avoidance and fuel cost reduction analysis summary.

\begin{tabular}{|c|c|c|c|c|c|}
\hline Mission & $\begin{array}{c}\text { Replacement } \\
\text { Model }\end{array}$ & $\begin{array}{l}\text { Extrapolated } \\
\text { U.S. Yearly } \\
\mathrm{CO}_{2} \mathrm{e} \text { Avoided } \\
\text { (lb- } \mathrm{CO}_{2} \mathrm{e} / \text { year)/ } \\
\% \text { reduction }\end{array}$ & $\begin{array}{l}\text { Extrapolated } \\
\text { Local Yearly } \\
\mathrm{CO}_{2} \mathrm{e} \mathrm{Avoided} \\
\left.\text { (lb- } \mathrm{CO}_{2} \mathrm{e} / \text { year }\right) \\
/ \% \text { reduction }\end{array}$ & $\begin{array}{l}\text { Extrapolated } \\
\text { U.S. Yearly } \\
\text { Fuel Cost } \\
\text { Reduction/ \% } \\
\text { reduction }\end{array}$ & $\begin{array}{c}\text { Extrapolated } \\
\text { Local Yearly } \\
\text { Fuel Cost } \\
\text { Reduction/ \% } \\
\text { reduction }\end{array}$ \\
\hline Support & Leaf & $2,579.6 / \mathbf{6 1 \%}$ & $3,281.4 / 78 \%$ & $\$ 620.51 / 82 \%$ & $\$ 635.55 / 86 \%$ \\
\hline Pool & Leaf & $6,844.6 / \mathbf{5 7 \%}$ & $9,093.8 / 75 \%$ & $\$ 1,732.36 / \mathbf{8 0} \%$ & $\$ 1,786.99 / \mathbf{8 4} \%$ \\
\hline Support & VTRUX PU & $3,701.5 / 42 \%$ & $5,879.8 / \mathbf{6 7 \%}$ & $\$ 1,150.39 / 73 \%$ & $\$ 1,216.56 / 79 \%$ \\
\hline Support & Rav4 & $11,916.6 / \mathbf{5 3} \%$ & $16,427.4 / 73 \%$ & $\$ 3,148.15 / 78 \%$ & $\$ 3,265.92 / \mathbf{8 3} \%$ \\
\hline Pool & Fusion & $4,011.8 / \mathbf{5 2} \%$ & $5,591.9 / 73 \%$ & $\$ 1,073.50 / 77 \%$ & $\$ 1,115.49 / \mathbf{8 3} \%$ \\
\hline Pool & Rav4 & $5,830.5 / 46 \%$ & $8,716.9 / \mathbf{6 9 \%}$ & $\$ 1,691.13 / 75 \%$ & $\$ 1,774.61 / \mathbf{8 0} \%$ \\
\hline Pool & Outlander & $6,326.3 / \mathbf{5 0} \%$ & $9,064.7 / 71 \%$ & $\$ 1,747.60 / 76 \%$ & $\$ 1,823.21 / \mathbf{8 2} \%$ \\
\hline
\end{tabular}

${ }^{33} \mathrm{http}: / /$ www.eia.gov/dnav/pet/pet_pri_gnd_dcus_sco_w.htm [accessed July 25, 2014].

${ }^{34} \mathrm{http}: / / w w w . e i a . g o v / e l e c t r i c i t y /$ state/ [Accessed July 25, 2014]. 


\begin{tabular}{|c|c|c|c|c|c|c|}
\hline & Mission & $\begin{array}{c}\text { Replacement } \\
\text { Model }\end{array}$ & $\begin{array}{c}\text { Extrapolated } \\
\text { U.S. Yearly } \\
\mathrm{CO}_{2} \mathrm{e} \text { Avoided } \\
\text { (lb-CO } \mathrm{CO}_{2} \mathrm{e} / \text { year)/ } \\
\% \text { reduction }\end{array}$ & $\begin{array}{l}\text { Extrapolated } \\
\text { Local Yearly } \\
\mathrm{CO}_{2} \mathrm{e} \mathrm{Avoided} \\
\text { (lb- } \mathrm{CO}_{2} \mathrm{e} / \text { year) } \\
/ \% \text { reduction }\end{array}$ & $\begin{array}{c}\text { Extrapolated } \\
\text { U.S. Yearly } \\
\text { Fuel Cost } \\
\text { Reduction/ \% } \\
\text { reduction }\end{array}$ & $\begin{array}{c}\text { Extrapolated } \\
\text { Local Yearly } \\
\text { Fuel Cost } \\
\text { Reduction/ \% } \\
\text { reduction }\end{array}$ \\
\hline & Support & VTRUX PU & $386.0 / \mathbf{1 7 \%}$ & $1,203.3 / \mathbf{5 2} \%$ & $\$ 250.96 / 61 \%$ & $\$ 280.33 / 79 \%$ \\
\hline & Pool & Outlander & $399.0 / \mathbf{1 3 \%}$ & $1,552.0 / \mathbf{5 0} \%$ & $\$ 327.85 / \mathbf{5 9 \%}$ & $\$ 369.94 / 68 \%$ \\
\hline & Support & Volt & $442.5 / \mathbf{1 7} \%$ & $1,342.5 / \mathbf{5 3} \%$ & $\$ 279.52 / 61 \%$ & $\$ 311.78 / 70 \%$ \\
\hline & Support & Outlander & $1,161.3 / \mathbf{3 3} \%$ & $2,171.5 / 62 \%$ & $\$ 433.48 / \mathbf{6 8 \%} \%$ & $\$ 466.68 / 76 \%$ \\
\hline \multirow{3}{*}{\multicolumn{3}{|c|}{$\begin{array}{l}\text { Total } \\
\text { Total Pool }\end{array}$}} & $43,600 / \mathbf{4 7} \%$ & $64,325 / 70 \%$ & $\$ 12,455 / 75 \%$ & $\$ 13,047 / \mathbf{8 1} \%$ \\
\hline & & & $23,412 / 49 \%$ & $34,019 / 71 \%$ & $\$ 6,572 / 76 \%$ & $\$ 6,870 / \mathbf{8 1} \%$ \\
\hline & & Total Support & $20,188 / \mathbf{4 6} \%$ & $30,306 / 69 \%$ & $\$ 5,883 / 75 \%$ & $\$ 6,177 / 80 \%$ \\
\hline
\end{tabular}

Table 19 shows the high potential benefit for reducing GHG emissions in the local GCNP area. This is primarily due to the heavy reliance on nuclear as the generating fuel (which also explains why the reduction experienced based on national figures is less than the Arizona reduction). In addition, the fuel cost reduction potential benefit is also significant due to the low local cost of power.

As presented in Section 5, 42 BEVs and 62 PHEVs could replace the pool fleet of 106 vehicles while retaining the two passenger vans. The support fleet of 109 vehicles would retain four heavy-duty pickups and four passenger vans and replace the balance with 34 BEVs and 67 PHEVs. Four BEVs and nine PHEVs are assumed to replace the enforcement fleet of 13 vehicles. Using an average savings per vehicle, Table 20 provides the avoided GHG and fuel cost savings should these replacements occur. Additional savings result if GCNP includes the portions of their fleet with other missions. The table also shows the percentage reduction in GHG emissions and fuel costs for ease of comparison. Only local Arizona savings are projected in Table 20.

Table 20. Extrapolated greenhouse gas emissions avoided and fuel cost savings for the entire fleet.

\begin{tabular}{|c|c|c|}
\hline Mission & $\begin{array}{c}\text { Extrapolated Local Yearly } \mathrm{CO}_{2} \mathrm{e} \\
\left.\text { Avoided (lb- } \mathrm{CO}_{2} \mathrm{e} / \text { year }\right) / \% \text { reduction }\end{array}$ & $\begin{array}{l}\text { Extrapolated Local Yearly Fuel Cost } \\
\text { Reduction (\$/year)/\% reduction }\end{array}$ \\
\hline Pool & $632,242 / \mathbf{6 8 \%}$ & $\$ 129,506 / \mathbf{8 0} \%$ \\
\hline Support & $734,435 / 70 \%$ & $\$ 148,616 / 81 \%$ \\
\hline Enforcement & $87,932 / \mathbf{6 9} \%$ & $\$ 17,922 / \mathbf{8 0} \%$ \\
\hline Total & $1,454,610 / \mathbf{6 9} \%$ & $\$ 296,044 / \mathbf{8 0} \%$ \\
\hline
\end{tabular}

\section{OBSERVATIONS}

Intertek appreciates the opportunity to present the results of this evaluation. Observations for possible follow-up action include the following:

\section{Observation \#1:}

Implementation: GCNP can move forward in the near future with the replacement of pool and support vehicles with PEVs as current budget and vehicle replacement schedules allow. Certainly, most of the vehicle types studied in this report are candidates for immediate replacement.

\section{Observation \#2:}

Fleet Inventory: A more thorough examination of the quantities and types of fleet vehicles within each usage category may be beneficial to quantify the potential for replacement by PEVs. While Intertek 
suggests a mix of BEVs and PHEVs, a more refined look may be possible. In addition, this study did not look at the other fleet vehicle categories such as specialty, transport vehicles, and shuttles/buses.

\section{Observation \#3:}

Vehicle Replacement Plan: The development of a detailed vehicle replacement plan could be beneficial. This plan would include cost and schedule for vehicle replacement. A more detailed survey and calculation of fleet vehicles use (such as vehicle parking locations, age of vehicle, expected replacement time, expected replacement costs, GSA vehicle costs, EVSE cost, total life costs, and EVSE installation costs) provide support to this replacement plan. A more refined estimate for reduced GHG emissions, petroleum usage reduction, and fuel cost savings flow from this detailed plan.

\section{Observation \#4:}

Infrastructure Planning: In conjunction with the replacement plan, evaluation of the GCNP sites for placement of PEV charging infrastructure could be beneficial. Intertek has significant experience in this area and such plans will consider not only fleet vehicle charging needs, but the convenience that charging infrastructure provides employees and visitors. This planning also considers the existing facility electrical distribution system. Vehicle home base considerations factor into the ratio of PEVs to EVSE units to maintain all vehicles at operational readiness.

Charging stations located at various destination points may provide additional infrastructure for PEV charging of the GCNP fleet. Charging stations at GCNP may also provide an opportunity for charging by the public. GCNP can benefit through collection of charging fees during times when these stations are not required for the overnight charging of fleet vehicles. The fees avoid the questions associated with a federal agency providing fuel for privately owned vehicles and support the costs for installation and operation of EVSE. 


\section{Appendix A, Fleet Survey Form}

\begin{tabular}{ll} 
& \multicolumn{1}{c}{ Fleet Survey Sample } \\
\hline Project Name: & BEA-FEMP Fleet II \\
Agency Name: & National Park Service \\
Location: & GCNP \\
Date Requested: & $2 / 2 / 2013$ \\
\hline
\end{tabular}

The following survey questions are used to lead the discussion concerning the mission of the current fleet of vehicles. If responding by e-mail, please use one form for each vehicle.

Please submit the data sheets to Ian Nienhueser at ian.nienhueser@intertek.com by fax at (602) 443-9007. If you have questions, please contact Ian at the email above or by phone at (702) $738-2706$.

\begin{tabular}{llll}
\hline \multicolumn{4}{c}{ Vehicle Information } \\
\hline Today's Date: & $2 / 17 / 2013$ & Odometer Reading: & 17,589 \\
Make: & Chevrolet & Data Logger ID: & 64 \\
Model: & Tahoe & Data Logger Installed: & $2 / 17 / 2013$ \\
Year: & 2012 & Fuel Type: & Gasoline \\
VIN: & 1GNSK2E01CR301102 & Miles per Gallon: & $23 / 31$ \\
Agency Fleet ID: & NA & Miles per Year: & 10,000 \\
\hline
\end{tabular}

\begin{tabular}{|c|c|l|}
\hline 1. & \multicolumn{2}{|l|}{ Vehicle Mission: } \\
\cline { 2 - 3 } & $\square$ & Pool Vehicle \\
\hline$\square$ & Enforcement Vehicle \\
\hline$\square$ & Support Vehicle \\
\hline$\square$ & Transport Vehicle \\
\hline$\square$ & Specialty Vehicle \\
\hline$\square$ & Shuttle/Bus \\
\hline & $\square$ & Low-Speed Vehicle \\
\hline \multirow{2}{*}{2.} & \multicolumn{2}{|l}{ Vehicle Typical Parking Location: (parking lot name/designation, nearest building number) } \\
\cline { 2 - 3 } & \multicolumn{2}{|}{} \\
\hline
\end{tabular}




\section{Appendix B, Definitions}

Alternative fuel

City fuel economy (MPG)

Conventional fuel

Daily travel

Diesel fuel

E85

Electric vehicle

Ethanol-fueled vehicle

Federal vehicle standards

Government motor vehicle

Gross vehicle weight rating

GSA fleet
An alternative fuel means any fuel other than gasoline and diesel fuels, such as methanol, ethanol, and gaseous fuels (40 CFR 86.1803-01). A fuel type other than petroleum-based gasoline or diesel as defined by the Energy Policy Act (examples include ethanol, methanol, compressed natural gas, propane, and electrical energy).

City fuel economy means the city fuel economy determined by operating a vehicle (or vehicles) over the driving schedule in the federal emission test procedure or determined according to the vehicle-specific 5-cycle or derived 5-cycle procedures (40 CFR 600.001).

A petroleum-based fuel (examples include gasoline and diesel fuel).

The sum of daily trips and stops in one day.

Diesel means a type of engine with operating characteristics significantly similar to the theoretical diesel combustion cycle. The non-use of a throttle during normal operation is indicative of a diesel engine (49 CFR 86-1803).

Ethanol fuel blend of up to $85 \%$ denatured ethanol fuel and gasoline or other hydrocarbons by volume.

Electric vehicle means a motor vehicle that is powered solely by an electric motor drawing current from a rechargeable energy storage system, such as from storage batteries or other portable electrical energy storage devices, including hydrogen fuel cells, provided that

(1) The vehicle is capable of drawing recharge energy from a source off the vehicle, such as residential electric service

(2) The vehicle must be certified to the emission standards of Bin \#1 of Table S04-1 in $\S 86.1811-09$ (c)(6)

(3) The vehicle does not have an onboard combustion engine/generator system as a means of providing electrical energy (40 CFR 86-1803).

Ethanol-fueled vehicle-means any motor vehicle or motor vehicle engine that is engineered and designed to be operated using ethanol fuel (i.e., a fuel that contains at least $50 \%$ ethanol $\left(\mathrm{C}_{2} \mathrm{H}_{5} \mathrm{OH}\right)$ by volume) as fuel (40 CFR 86.1803-01).

The document that establishes classifications for various types and sizes of vehicles, general requirements, and equipment options. It is issued annually by the GSA Vehicle Acquisition and Leasing Service's Automotive Division.

Any motor vehicle that the government owns or leases. This includes motor vehicles obtained through purchase, excess, forfeiture, commercial lease, or GSA fleet lease.

Gross vehicle weight rating (GVWR) means the value specified by the vehicle manufacturer as the maximum design loaded weight of a single vehicle (e.g., vocational vehicle) (US Government Printing Office 2009)

GSA fleet lease means obtaining a motor vehicle from the General Services Administration fleet (GSA fleet) (41 CFR 102-34). 
Heavy light-duty truck

Highway fuel economy (Hwy MPG)

Hybrid electric vehicle

Idle time

Law enforcement

Light-duty motor vehicle Light-duty truck
Heavy light-duty truck means any light-duty truck rated greater than 6,000 lb GVWR. The light-duty truck 3 (LDT3) and LDT4 classifications comprise the heavy light-duty truck category (40 CFR 86.1803-01).

Highway fuel economy means the highway fuel economy determined either by operating a vehicle (or vehicles) over the driving schedule in the federal highway fuel economy test procedure or determined according to either the vehicle-specific, 5-cycle equation, or the derived 5-cycle equation for highway fuel economy (40 CFR 600.001).

Hybrid electric vehicle means a motor vehicle that draws propulsion energy from onboard sources of stored energy that are both an internal combustion engine or heat engine using consumable fuel and a rechargeable energy storage system (such as a battery, capacitor, hydraulic accumulator, or flywheel), where recharge energy for the energy storage system comes solely from sources on board the vehicle.

Idle time is logged whenever a vehicle idles with the engine running for 3 minutes or longer.

Law enforcement motor vehicle means a light-duty motor vehicle that is specifically approved in an agency-s appropriation act for use in apprehension, surveillance, police, or other law enforcement work or specifically designed for use in law enforcement. If not identified in an agency's appropriation language, a motor vehicle qualifies as a law enforcement motor vehicle only in the following cases:

(1) A passenger automobile having heavy-duty components for electrical, cooling, and suspension systems and at least the next higher cubic inch displacement or more powerful engine than is standard for the automobile concerned

(2) A light truck having emergency warning lights and identified with markings such as "police"

(3) An unmarked motor vehicle certified by the agency head as essential for the safe and efficient performance of intelligence, counterintelligence, protective, or other law enforcement duties

(4) A forfeited motor vehicle seized by a federal agency that subsequently is used for performing law enforcement activities (41 CFR Part 102-34.35).

Any motor vehicle with a GVWR of 8,500 pounds or less (41 CFR 102-34). Light-duty truck means any motor vehicle rated at 8,500 pounds GVWR or less, which has a curb weight of 6,000 pounds or less and, which has a basic vehicle frontal area of 45 square feet or less, which is as follows:

(1) Designed primarily for purposes of transportation of property or is a derivation of such a vehicle

(2) Designed primarily for transportation of persons and has a capacity of more than 12 persons

(3) Available with special features, enabling off-street or off-highway operation and use.

LDT1 means any light light-duty truck up through 3,750-lb loaded vehicle weight.

LDT2 means any light light-duty truck greater than 3,750-lb loaded vehicle weight. 


\section{Light-duty vehicle \\ Low-speed vehicle \\ Light-duty vehicle means a passenger car or passenger car derivative capable of seating 12 passengers or less. \\ Low-speed vehicle means a motor vehicle \\ (1) That is 4-wheeled \\ (2) Whose speed attainable in $1.6 \mathrm{~km}$ (1 mile) is more than 32 kilometers per hour (20 miles per hour) and not more than 40 kilometers per hour (25 miles per hour) on a paved level surface \\ (3) Whose GVWR is less than 1,361 kilograms (3,000 pounds) (49 CFR 571.3 - Definitions).}

Medium-duty passenger vehicle

Model year

$M P G$

$M P G e$

Non-passenger automobile
LDT3 means any heavy light-duty truck up through 5,750-lb adjusted loaded vehicle weight.

LDT4 means any heavy light-duty truck greater than 5,750-lb adjusted loaded vehicle weight (US Government Printing Office 2009)

Medium-duty passenger vehicle means any heavy-duty vehicle (as defined in this subpart) with a GVWR of less than 10,000 pounds that is designed primarily for transportation of persons. The medium-duty passenger vehicle definition does not include any vehicle which

(1) Is an "incomplete truck" as defined in this subpart

(2) Has a seating capacity of more than 12 persons

(3) Is designed for more than 9 persons in seating rearward of the driver's seat

(4) Is equipped with an open cargo area (for example, a pick-up truck box or bed) of 72.0 inches in interior length or more. A covered box not readily accessible from the passenger compartment will be considered an open cargo area for purposes of this definition (US Government Printing Office 2009)

Model year means the manufacturer's annual production period (as determined by the administrator), which includes January 1 of such calendar year; provided that if the manufacturer has no annual production period, the term "model year" shall mean the calendar year (40 CFR 86-1803.01).

"MPG" or "mpg" means miles per gallon. This generally may be used to describe fuel economy as a quantity or it may be used as the units associated with a particular value.

MPGe means miles per gallon equivalent. This generally is used to quantify a fuel economy value for vehicles that use a fuel other than gasoline. The value represents miles the vehicle can drive with the energy equivalent of one gallon of gasoline:

(c) SCF means standard cubic feet

(d) SUV means sport utility vehicle

(e) CREE means carbon-related exhaust emissions [76 FR 39527, July 6, 2011].

A non-passenger automobile means an automobile that is not a passenger automobile or a work truck and includes vehicles described in paragraphs (a) and (b) of 49 CFR 523.5. 
Owning agency

Passenger automobile

Pickup truck

Plug-in hybrid electric vehicle

Vehicle class

Vehicle configuration

Vehicle days

Vehicle home base

Vehicle study period
Owning agency means the executive agency that holds the vehicle title, manufacturer's Certificate of Origin or is the lessee of a commercial lease. This term does not apply to agencies that lease motor vehicles from the GSA fleet (41 CFR Part 102-34.35).

A passenger automobile is any automobile (other than an automobile capable of off-highway operation) manufactured primarily for use in the transportation of not more than 10 individuals (49 CFR 523.4 - Passenger automobile). A sedan or station wagon designed primarily to transport people (41 CFR 102-34).

Pickup truck means a non-passenger automobile, which has a passenger compartment and an open cargo bed (49 CFR 523.2).

PHEV means a hybrid electric vehicle that has the capability to charge the battery from an off-vehicle electric source, such that the off-vehicle source cannot be connected to the vehicle while the vehicle is in motion (40 CFR 86.1803).

The designation of motor vehicle types that include sedans, station wagons, ambulances, buses, and trucks, or different categories of vehicles according to Federal vehicle standards and further defined in 49 CFR 600.315-82.

Vehicle configuration means a unique combination of basic engine, engine code, inertia weight class, transmission configuration, and axle ratio.

The number of days a vehicle was driven or utilized during the (vehicle) study period.

The primary assigned outing beginning and ending parking location for the vehicle.

The time period the vehicle, within the study, has been equipped with a data logger. 


\section{Appendix C, Grand Canyon National Park Map}

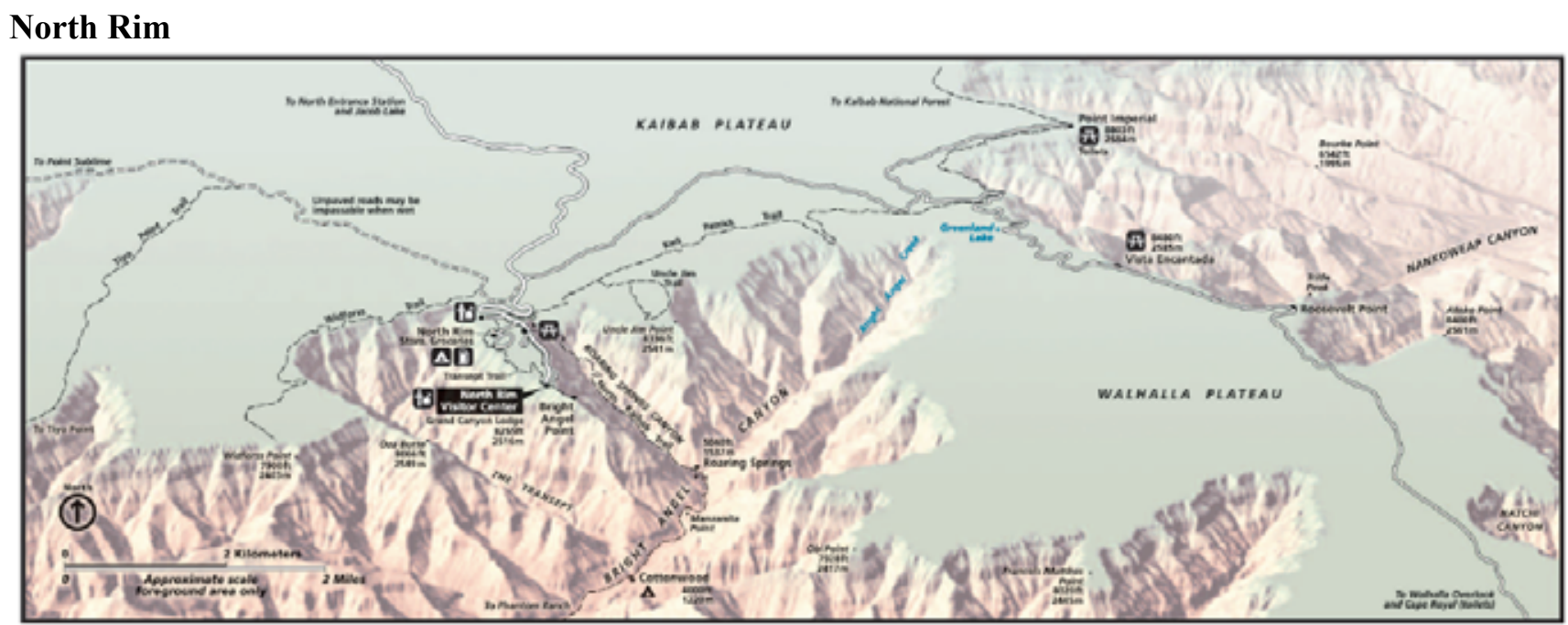

\section{South Rim}

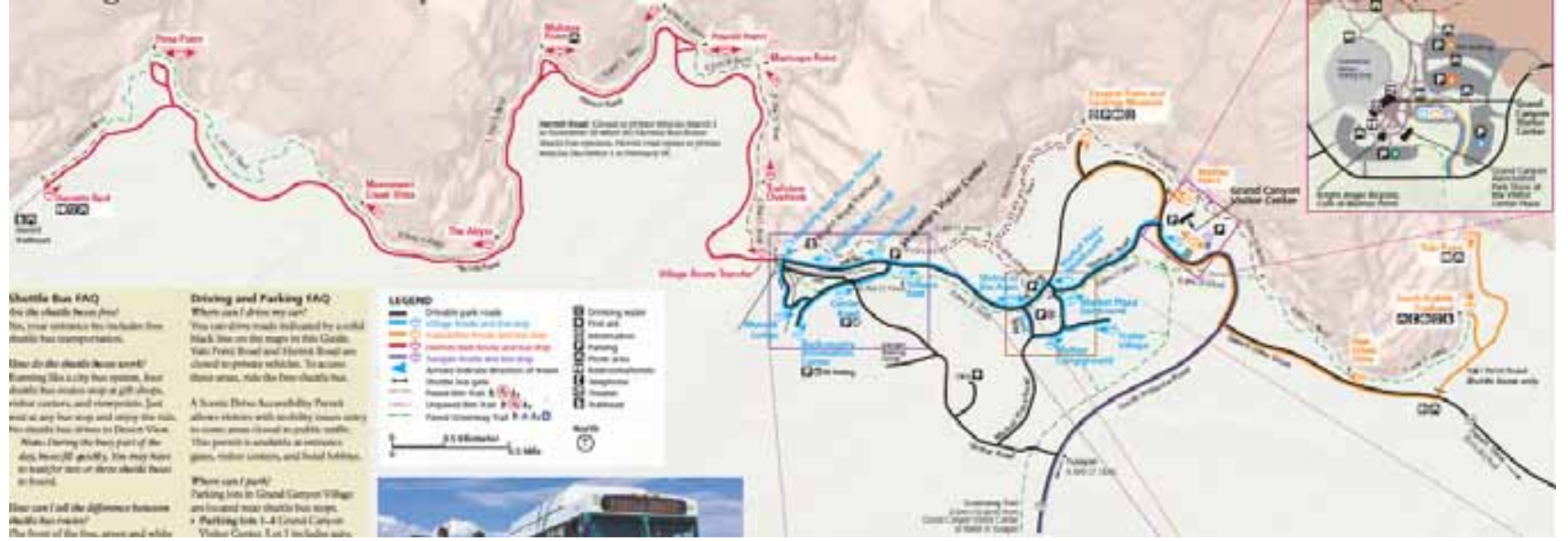

\section{Desert View Drive}

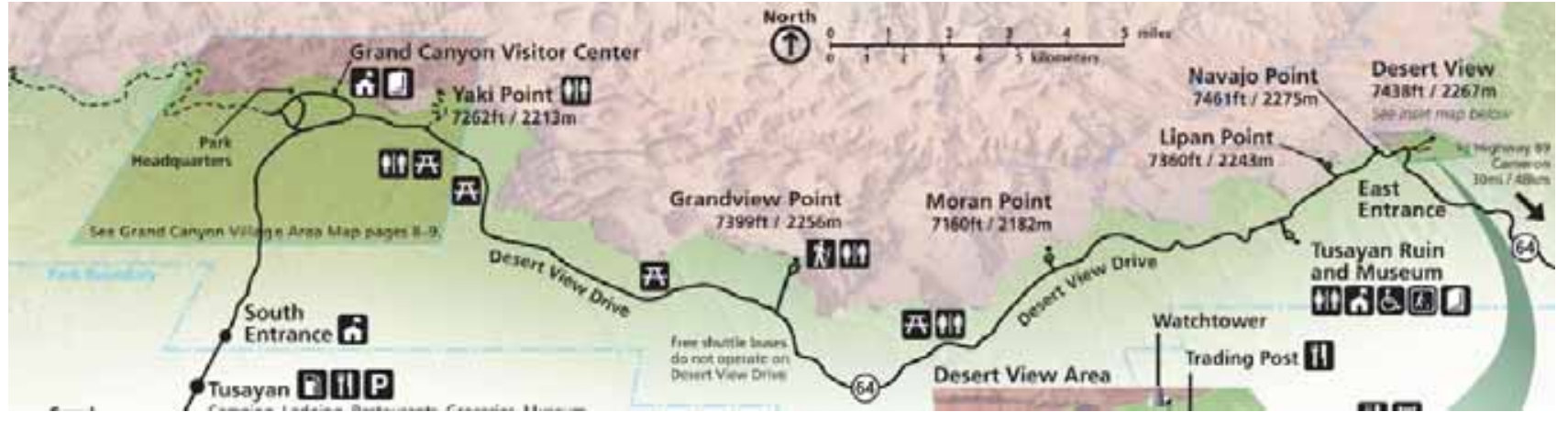




\section{Appendix D, Vehicle Characterization}

Table D-1. Grand Canyon National Park vehicle index.

\begin{tabular}{|c|c|c|c|c|c|c|}
\hline \multicolumn{7}{|c|}{ Vehicle Index } \\
\hline $\log$ & Fleet Vehicle Id & Make & Model & Year & EPA Class & Mission \\
\hline 67 & 42612 & Jeep & Liberty & 2006 & SUV & Support \\
\hline 68 & 79736 & Chevrolet & Uplander & 2008 & Minivan & Pool \\
\hline 69 & 70762 & Ford & $\mathrm{F} 250$ & 2009 & Pickup Truck & Support \\
\hline 70 & 70202 & Ford & F250 & 2011 & Pickup Truck & Support \\
\hline 71 & 38768 & Chevrolet & Impala & 2010 & Sedan-Large & Pool \\
\hline 72 & 67912 & Ford & Explorer & 2010 & SUV & Pool \\
\hline 73 & 45115 & Dodge & Durango & 2008 & SUV & Pool \\
\hline 74 & 90661 & Ford & Ranger & 2007 & Pickup Truck & Support \\
\hline 75 & 20389 & Chevrolet & HHR & 2010 & SUV & Pool \\
\hline 79 & 17523 & Ford & Focus & 2011 & Sedan-Compact & Support \\
\hline 80 & 69685 & Ford & Escape & 2011 & SUV & Support \\
\hline
\end{tabular}




\section{Appendix E, \\ GCNP Vehicle Data Sheets}

\section{Vehicle 42612}

\begin{tabular}{|c|c|c|c|c|}
\hline & \multicolumn{2}{|c|}{ Make/Model/Year } & \multicolumn{2}{|c|}{ Jeep/Liberty/2006 } \\
\hline$=2-12$ & \multicolumn{2}{|l|}{ EPA Class Size } & \multicolumn{2}{|c|}{ SUV } \\
\hline$=8$ & \multicolumn{2}{|l|}{ Mission } & \multicolumn{2}{|c|}{ Support } \\
\hline & \multicolumn{2}{|l|}{ VIN } & \multicolumn{2}{|c|}{ 1J4GL48K76W242612 } \\
\hline & \multicolumn{2}{|c|}{ Parking Location } & \multicolumn{2}{|c|}{ Mail Room } \\
\hline & \multicolumn{2}{|c|}{ Fleet Vehicle ID (VIN5) } & \multicolumn{2}{|c|}{42612} \\
\hline & \multicolumn{2}{|l|}{ Fuel Type } & \multicolumn{2}{|c|}{ Gas } \\
\hline & \multicolumn{2}{|c|}{ EPA Label/MPG (City/Hwy/Combined) } & \multicolumn{2}{|c|}{$16 / 20 / 17$} \\
\hline & \multicolumn{2}{|c|}{ EPA GHG Emissions (Grams $\mathrm{CO}_{2} / \mathrm{Mi}$ ) } & \multicolumn{2}{|c|}{523} \\
\hline & \multicolumn{2}{|l|}{ Study Logger ID } & \multicolumn{2}{|c|}{ Logger 67} \\
\hline & \multicolumn{2}{|c|}{ Total Vehicle Days/Total Study Days } & \multicolumn{2}{|c|}{$48 / 68$} \\
\hline \multicolumn{5}{|c|}{ Vehicle 42612 Travel Summary } \\
\hline & $\begin{array}{c}\text { Per Day } \\
\text { Average/Peak }\end{array}$ & $\begin{array}{c}\text { Per Outing } \\
\text { Average/Peak }\end{array}$ & $\begin{array}{c}\text { Per Trip } \\
\text { Average/Peak }\end{array}$ & Total \\
\hline Travel Distance (Miles) & $7.4 / 16.5$ & $2.3 / 8.8$ & $0.6 / 4.5$ & 335 \\
\hline Travel Time (Minutes) & $43 / 81.0$ & $13.3 / 60.0$ & $3.3 / 55.0$ & 2,046 \\
\hline Idle Time (Minutes) & $5.5 / \mathrm{NA}$ & $1.7 / \mathrm{NA}$ & $0.4 / \mathrm{NA}$ & 264 \\
\hline
\end{tabular}

\begin{tabular}{|c|c|c|c|c|}
\hline \multicolumn{2}{|c|}{ Total Stops } & \multicolumn{2}{c|}{ Stop Duration } \\
\hline $\begin{array}{c}\text { Distance From } \\
\text { Home Base (Miles) }\end{array}$ & Stops & Percentages & Stop Duration (Hours) & Stops \\
\hline Less than 10 & 425 & $100 \%$ & Less than 2 & 365 \\
\hline 10 to 20 & 0 & $0 \%$ & 2 to 4 & 18 \\
\hline 20 to 40 & 0 & $0 \%$ & 4 to 8 & 0 \\
\hline 40 to 60 & 0 & $0 \%$ & Greater than 8 & 42 \\
\hline
\end{tabular}

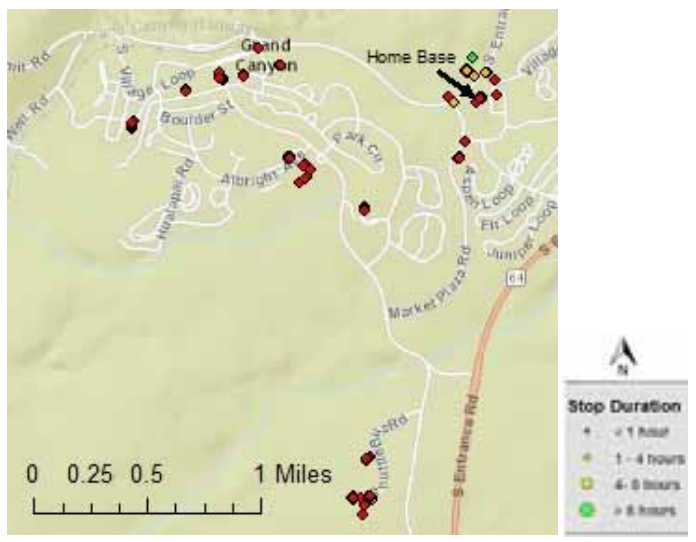

Figure D-1. Vehicle 42612 stops.

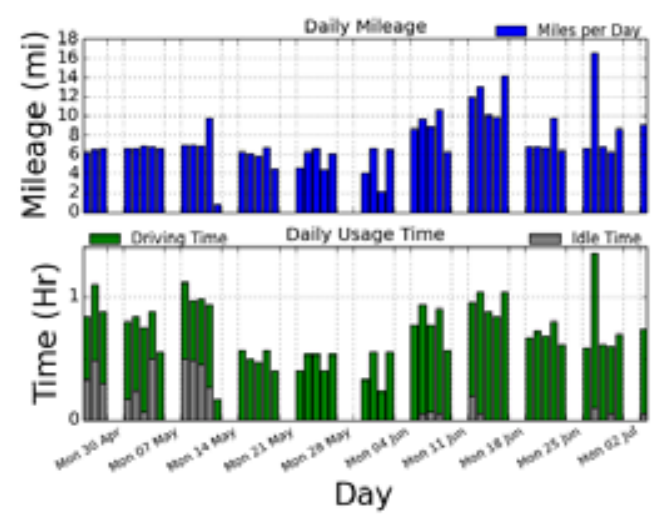

Figure D-2. Vehicle 42612 history. 

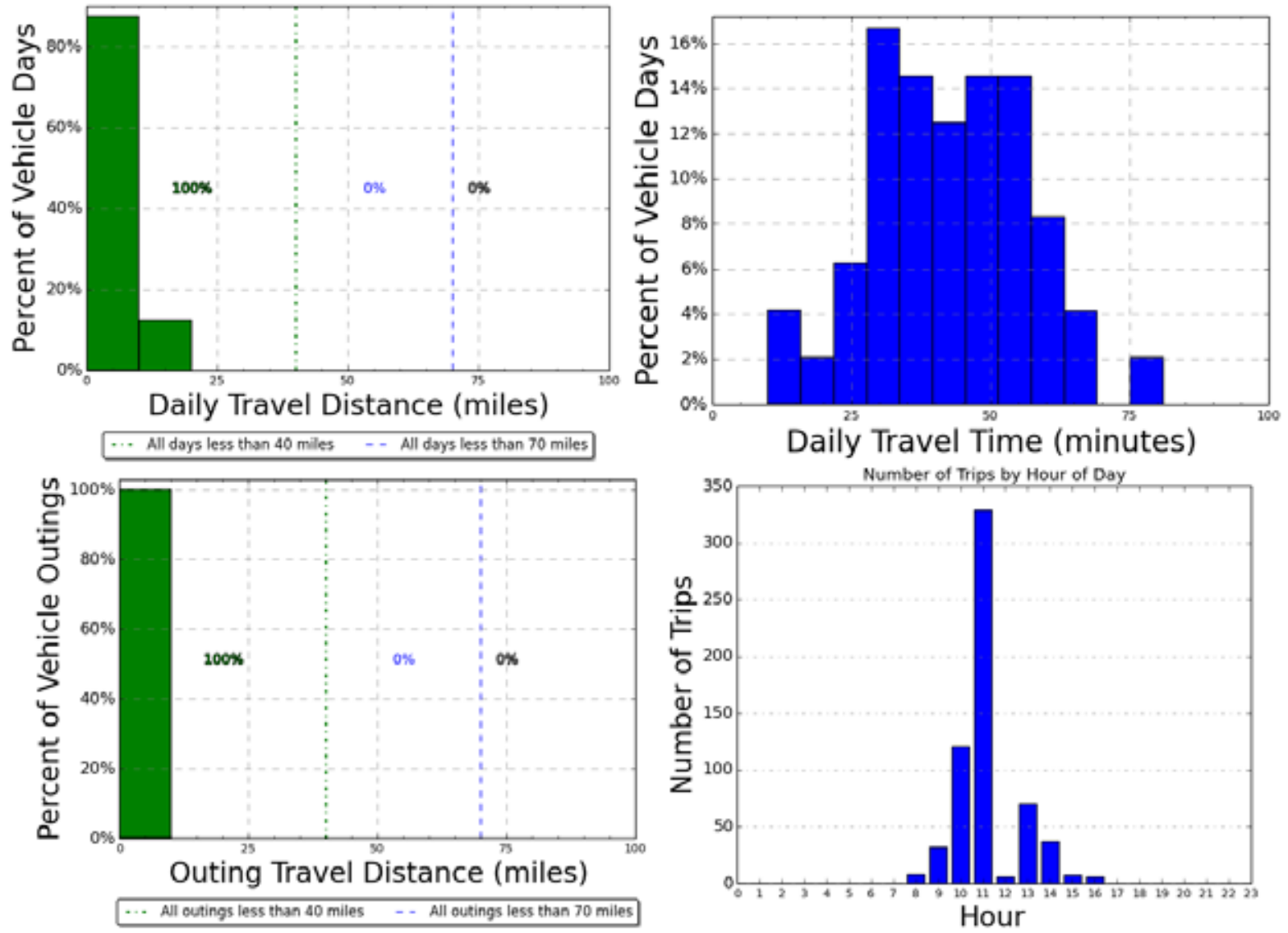

Figure D-3. Vehicle 42612 travel graphs.

\section{Vehicle 42612 Observations}

Logger 67 collected data on this vehicle for a period of 48 days of the 63-day study period. Validation occurred on $99.7 \%$ of the input data. Information provided on the vehicle survey for this vehicle indicated that this is a support vehicle typically used year around for park mail delivery and at the mailroom. Vehicle data indicate that this vehicle parks overnight on the South Entrance Road in Grand Canyon village.

The reported odometer reading was 21,397 miles, with an estimated usage of 3,566 miles/year.

All stops exceeding 2-hour duration occurred at the home base. The longest single trip and outing were within the typically advertised range of a BEV, which is approximately 70 miles. The longest day's travel also was within the advertised range of a BEV.

A BEV may provide acceptable performance for this vehicle should the BEV meet other mission requirements. The survey information suggests no other special requirements exist. BEVs currently are available as potential replacement for this vehicle. 


\begin{tabular}{|l|l|c|}
\hline Make/Model/Year & Chevrolet/Uplander/2008 \\
\hline EPA Class Size & Minivan \\
\hline Mission & Pool \\
\hline VIN & South Entrance Road \\
\hline Parking Location & 79736 \\
\hline Fleet Vehicle ID (VIN5) & Gas \\
\hline Fuel Type & $16 / 23 / 19$ \\
\hline EPA Label/MPG (City/Hwy/Combined) & 468 \\
\hline EPA GHG Emissions (Grams CO $/$ Mi) & Logger 68 \\
\hline & Study Logger ID & $71 / 87$ \\
\hline
\end{tabular}

\begin{tabular}{|l|c|c|c|c|}
\hline \multicolumn{5}{|c|}{ Vehicle 79736 Travel Summary } \\
\hline & $\begin{array}{c}\text { Per Day } \\
\text { Average/Peak }\end{array}$ & $\begin{array}{c}\text { Per Outing } \\
\text { Average/Peak }\end{array}$ & $\begin{array}{c}\text { Per Trip } \\
\text { Average/Peak }\end{array}$ & Total \\
\hline Travel Distance (Miles) & $13.8 / 399.9$ & $7.6 / 399.1$ & $2.3 / 124.2$ & 980 \\
\hline Travel Time (Minutes) & $39 / 451.0$ & $21.3 / 51.0$ & $6.4 / 132$ & 2,752 \\
\hline Idle Time (Minutes) & $1.7 / \mathrm{NA}$ & $0.9 / \mathrm{NA}$ & $0.3 / \mathrm{NA}$ & 119 \\
\hline
\end{tabular}

\begin{tabular}{|c|c|c|c|c|}
\hline \multicolumn{2}{|c|}{ Total Stops } & \multicolumn{2}{c|}{ Stop Duration } \\
\hline $\begin{array}{c}\text { Distance From } \\
\text { Home Base (Miles) }\end{array}$ & Stops & Percentages & Stop Duration (Hours) & Stops \\
\hline Less than 10 & 376 & $98.9 \%$ & Less than 2 & 250 \\
\hline 10 to 20 & 2 & $0.5 \%$ & 2 to 4 & 42 \\
\hline 20 to 40 & 0 & $0 \%$ & 4 to 8 & 21 \\
\hline 40 to 60 & 2 & $0.5 \%$ & Greater than 8 & 67 \\
\hline
\end{tabular}

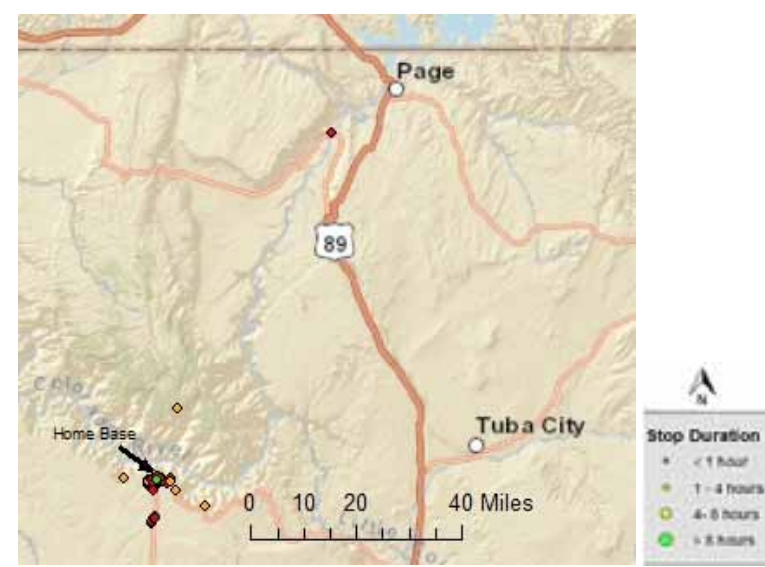

Figure D-4. Vehicle 79736 stops.

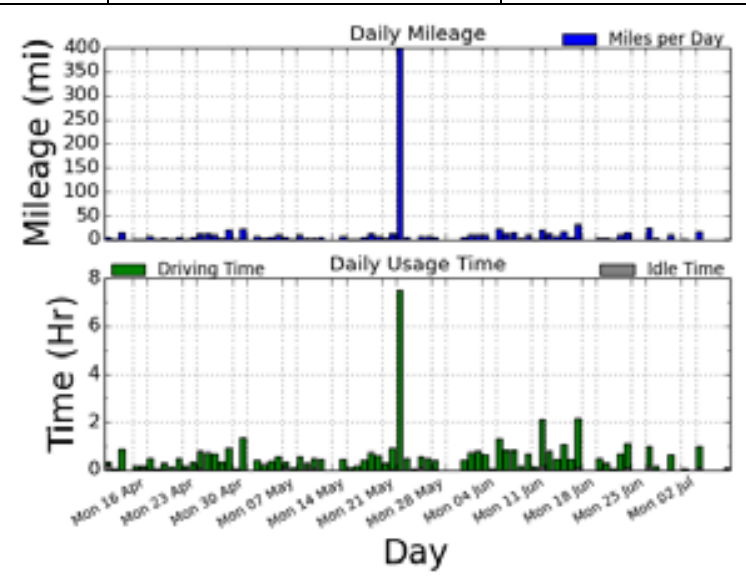

Figure D-5. Vehicle 79736 history. 

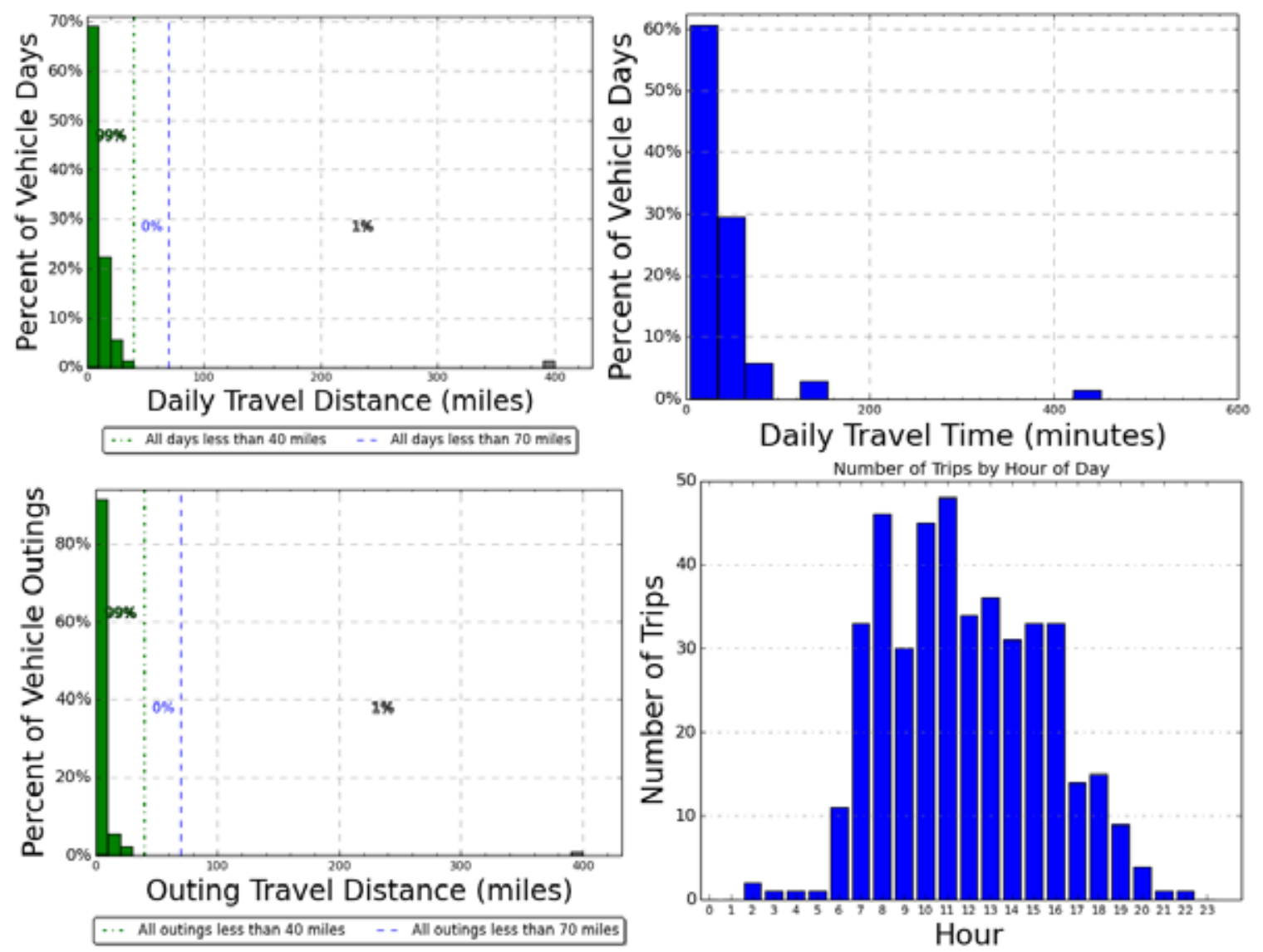

Figure D-4. Vehicle 79736 travel graphs.

\section{Vehicle 79736 Observations}

Logger 68 collected data on this vehicle for a period of 71 of the 87 study days. Validation occurred on $99.8 \%$ of the vehicle data. Survey information on this vehicle indicates it is used by the Division of Interpretation and Resource Education for trips in the park, to Flagstaff, and other travel. A pool mission is implied. Vehicle stop data indicate that this vehicle parks overnight primarily on South Entrance Road in Grand Canyon Village.

The reported odometer reading was 45,715 miles, with an estimated usage of 11,429 miles/year.

Ninety-nine percent of this vehicle's travel is less than 40 miles per day. One trip occurred on May 21, 2013, on a round-trip to Kaibab, Arizona and the North Rim. The maximum outing and daily travel coincide with this trip.

Aside from this one outing, a BEV may provide the acceptable performance of this minivan should the BEV meet other mission requirements. Information is unavailable suggesting special requirements exist. Daily travel requirements also allow the use of a PHEV to travel all day on CD mode power, thus providing an alternative for this vehicle. A BEV may be a suitable replacement for the fleet if PHEVs are available for longer trips. 
Vehicle 70762

\begin{tabular}{|c|c|c|c|c|}
\hline$=$ & \multicolumn{2}{|c|}{ Make/Model/Year } & \multicolumn{2}{|c|}{ Ford/F250/2009 } \\
\hline$x-x=1 \equiv$ & \multicolumn{2}{|l|}{ EPA Class Size } & \multicolumn{2}{|c|}{ Pickup Truck } \\
\hline $8 \ln =2 \mathrm{a}$ & \multicolumn{2}{|l|}{ Mission } & \multicolumn{2}{|c|}{ Support } \\
\hline & \multicolumn{2}{|l|}{ VIN } & \multicolumn{2}{|c|}{ IFTSW21569EA70762 } \\
\hline & \multicolumn{2}{|c|}{ Parking Location } & \multicolumn{2}{|c|}{ Roads } \\
\hline & \multicolumn{2}{|c|}{ Fleet Vehicle ID (VIN5) } & \multicolumn{2}{|c|}{70762} \\
\hline & \multicolumn{2}{|l|}{ Fuel Type } & \multicolumn{2}{|c|}{ Gas } \\
\hline & \multicolumn{2}{|c|}{ EPA Label/MPG (City/Hwy/Combined)* } & \multicolumn{2}{|c|}{$14 / 19 / 16$} \\
\hline & \multicolumn{2}{|c|}{ EPA GHG Emissions (Grams $\left.\mathrm{CO}_{2} / \mathrm{Mi}\right)^{*}$} & \multicolumn{2}{|c|}{523} \\
\hline & \multicolumn{2}{|c|}{ Study Logger ID } & \multicolumn{2}{|c|}{ Logger 69} \\
\hline & \multicolumn{2}{|c|}{ Total Vehicle Days/Total Study Days } & \multicolumn{2}{|c|}{$51 / 70$} \\
\hline \multicolumn{5}{|c|}{ Vehicle 70762 Travel Summary } \\
\hline & $\begin{array}{c}\text { Per Day } \\
\text { Average/Peak }\end{array}$ & $\begin{array}{c}\text { Per Outing } \\
\text { Average/Peak }\end{array}$ & $\begin{array}{c}\text { Per Trip } \\
\text { Average/Peak }\end{array}$ & Total \\
\hline Travel Distance (Miles) & $37.4 / 284.2$ & $10.8 / 284.0$ & $4.6 / 136.8$ & 1,909 \\
\hline Travel Time (Minutes) & $141 / 355.0$ & $40.7 / 338.0$ & $17.1 / 290.0$ & 7,202 \\
\hline Idle Time (Minutes) & $55.4 / \mathrm{NA}$ & $16.0 / \mathrm{NA}$ & 6.7/NA & 2,825 \\
\hline
\end{tabular}

\begin{tabular}{|c|c|c|c|c|}
\hline \multicolumn{2}{|c|}{ Total Stops } & \multicolumn{2}{c|}{ Stop Duration } \\
\hline $\begin{array}{c}\text { Distance From } \\
\text { Home Base (Miles) }\end{array}$ & Stops & Percentages & Stop Duration (Hours) & Stops \\
\hline Less than 10 & 416 & $93.9 \%$ & Less than 2 & 362 \\
\hline 10 to 20 & 24 & $5.4 \%$ & 2 to 4 & 26 \\
\hline 20 to 40 & 0 & $0 \%$ & 4 to 8 & 7 \\
\hline 40 to 60 & 3 & $0.7 \%$ & Greater than 8 & 48 \\
\hline
\end{tabular}

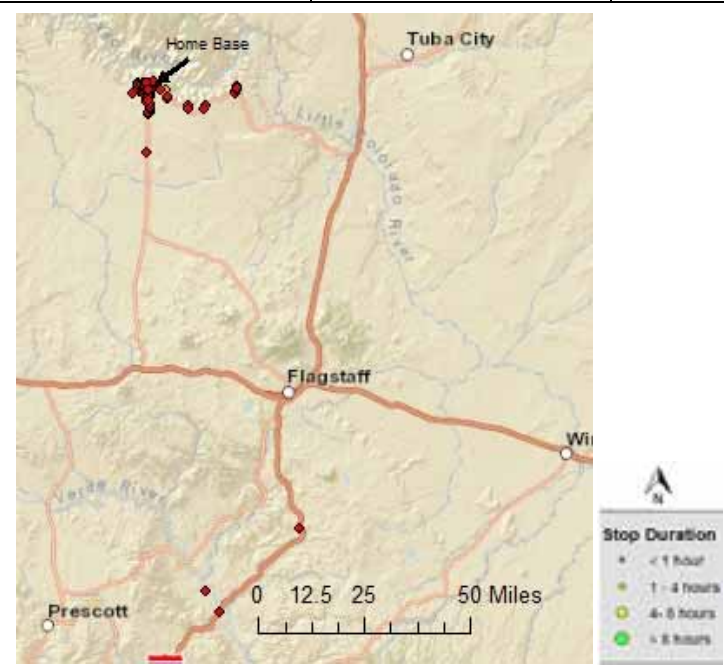

Figure D-7. Vehicle 70762 stops.

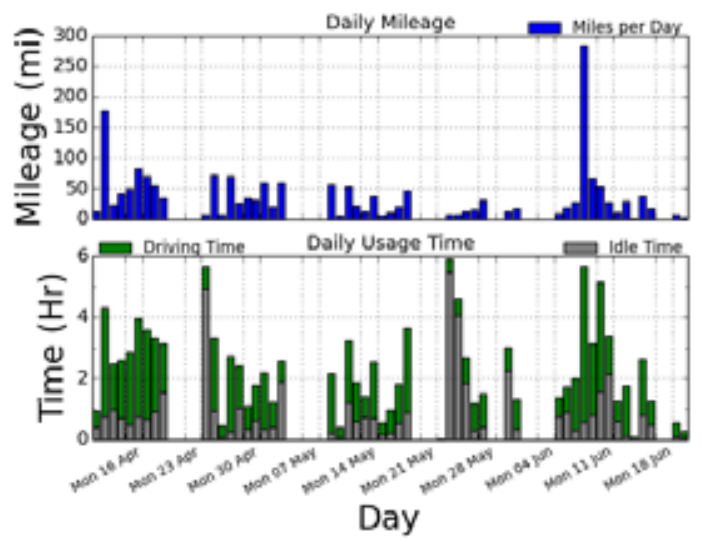

Figure D-8. Vehicle 70762 history.

*2009 Ford F150 truck information. EPA figures for 2009 Ford F250 not available. 

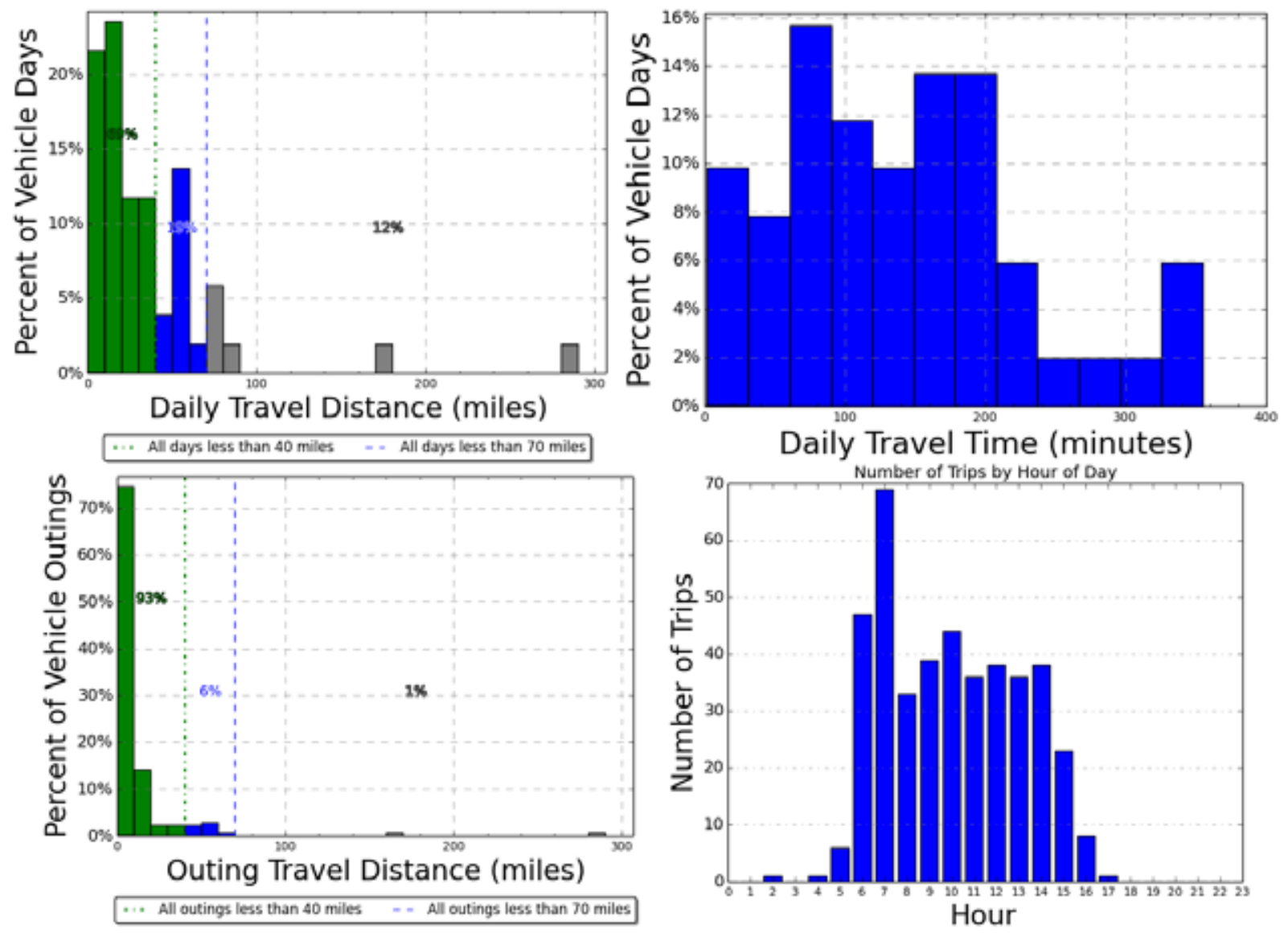

Figure D-5. Vehicle 70762 travel graphs.

\section{Vehicle 70762 Observations}

Logger 69 collected data on this vehicle for a period of 51 of the 70 study days. Validation occurred on $99.5 \%$ of the vehicle data. Vehicle survey information on this vehicle indicated it is a work truck for roadwork (e.g., painting, fixing holes, construction, etc.) and assigned to the Roads group of the Division of Facility Management. A support function is implied. Vehicle stop data indicate that this vehicle parks overnight primarily on Sunset Drive in Grand Canyon Village, although other overnight stops occurred on South Entrance Road and Albright Avenue in Grand Canyon Village.

The reported odometer reading was 30,394 miles, with an estimated usage of 10,131 miles/year.

Sixty-nine percent of daily travel is less than 40 miles and $88 \%$ is less than 70 miles. Most of vehicle travel is of relatively short distances, interrupted by two longer travel excursions. The two longest days occurred on April 11 at 177.9 miles and on June 7 at 284.2 miles. The longest single daily travel was an outing that included Camp Verde, Arizona as a destination.

A BEV may provide acceptable performance for $99 \%$ of this vehicle's outings and $88 \%$ of this vehicle's daily travel should a BEV meet other mission requirements. The use of this vehicle for roadwork suggests that the cargo capabilities of a pickup are required. Daily travel requirements allow the use of a PHEV, which would handle $93 \%$ of the outings and $69 \%$ of daily travel in CD mode; therefore, it provides an alternative for this vehicle. 


\begin{tabular}{|l|l|c|}
\hline & Make/Model/Year & Ford/F250/2011 \\
\hline EPA Class Size & Pickup Truck \\
\hline Mission & Support \\
\hline VIN & IFT7W2B68BEA70202 \\
\hline Parking Location & Roads \\
\hline Fleet Vehicle ID (VIN5) & 70202 \\
\hline Fuel Type & Gas \\
\hline EPA Label/MPG (City/Hwy)* & $13 / 18 / 14$ \\
\hline & EPA GHG Emissions (Grams $\left.\mathrm{CO}_{2} / \mathrm{Mi}\right)^{*}$ & 635 \\
\hline & Study Logger ID & Logger 70 \\
\hline & Total Vehicle Days/Total Study Days & $43 / 70$ \\
\hline
\end{tabular}

\begin{tabular}{|l|c|c|c|c|}
\hline \multicolumn{5}{|c|}{ Vehicle 70202Travel Summary } \\
\hline & $\begin{array}{c}\text { Per Day } \\
\text { Average/Peak }\end{array}$ & $\begin{array}{c}\text { Per Outing } \\
\text { Average/Peak }\end{array}$ & $\begin{array}{c}\text { Per Trip } \\
\text { Average/Peak }\end{array}$ & Total \\
\hline Travel Distance (Miles) & $36.7 / 112.7$ & $12.2 / 92.2$ & $4.4 / 51.1$ & 1,578 \\
\hline Travel Time (Minutes) & $187 / 439.0$ & $62.3 / 366.0$ & $22.5 / 192.0$ & 8,031 \\
\hline Idle Time (Minutes) & $61.9 / \mathrm{NA}$ & $20.6 / \mathrm{NA}$ & $7.5 / \mathrm{NA}$ & 2,662 \\
\hline
\end{tabular}

\begin{tabular}{|c|c|c|c|c|}
\hline \multicolumn{2}{|c|}{ Total Stops } & \multicolumn{2}{c|}{ Stop Duration } \\
\hline $\begin{array}{c}\text { Distance From } \\
\text { Home Base (Miles) }\end{array}$ & Stops & Percentages & Stop Duration (Hours) & Stops \\
\hline Less than 10 & 376 & $93.5 \%$ & Less than 2 & 343 \\
\hline 10 to 20 & 26 & $6.5 \%$ & 2 to 4 & 10 \\
\hline 20 to 40 & 0 & $0 \%$ & 4 to 8 & 6 \\
\hline 40 to 60 & 0 & $0 \%$ & Greater than 8 & 43 \\
\hline
\end{tabular}

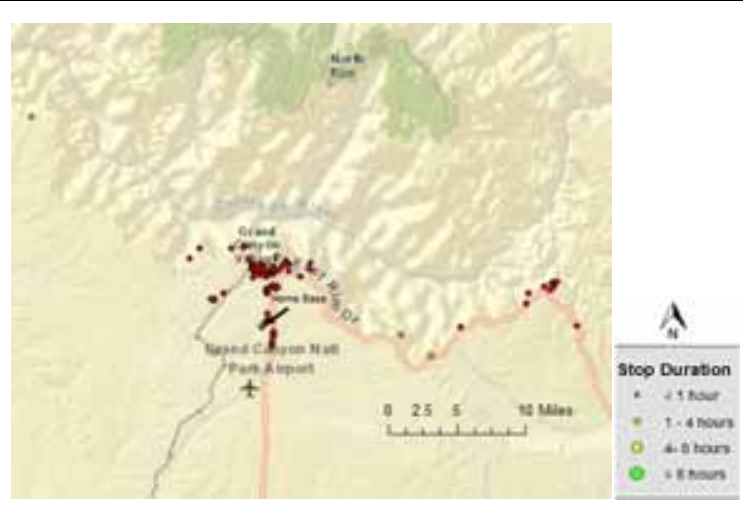

Figure D-10. Vehicle 70202 stops.

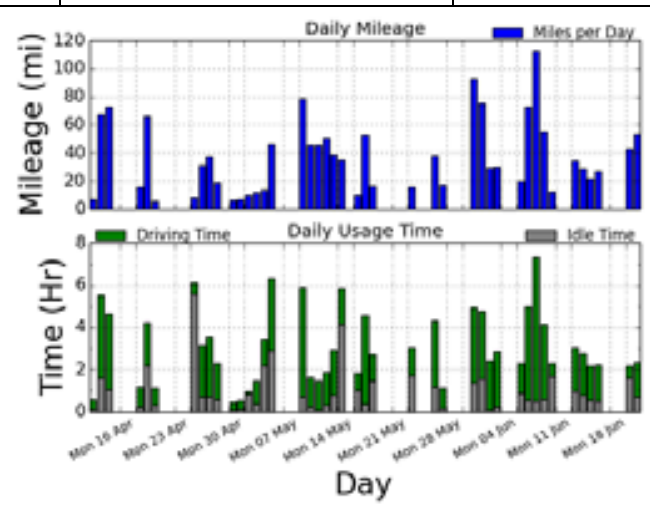

Figure D-11. Vehicle 70202 history.

*2011 Ford F150 truck information. EPA figures for 2011 Ford F250 not available. 

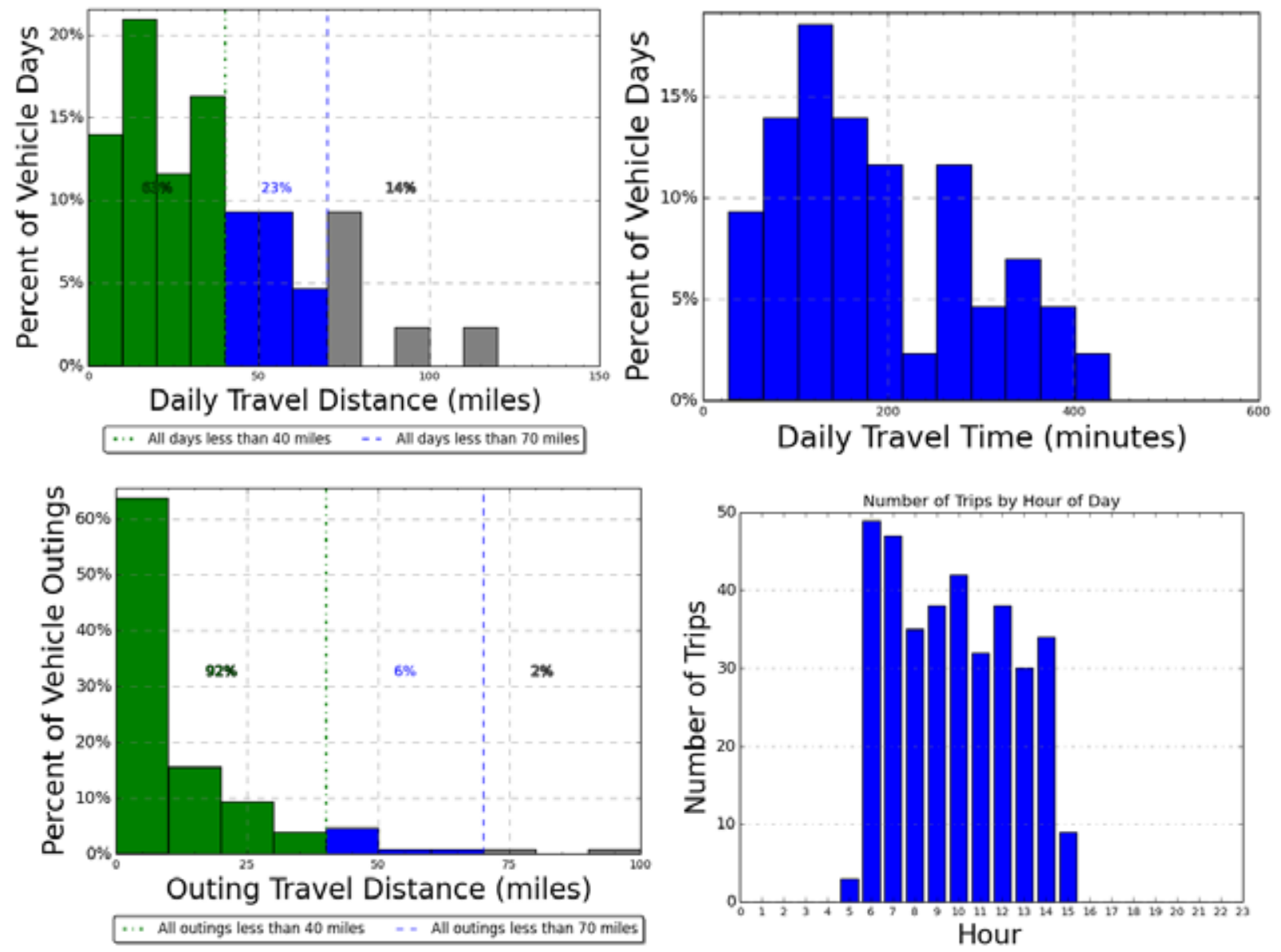

Figure D-6. Vehicle 70202 travel graphs.

\section{Vehicle 70202 Observations}

Logger 70 collected data on this vehicle for a period of 43 of the 70 study days. Validation occurred on $98.9 \%$ of the vehicle data. Vehicle survey information on this vehicle indicated it is a work truck for roadwork (e.g., painting, fixing holes, and construction) and is assigned to the Roads group of the Division of Facility Management. A support function is implied.

The reported odometer reading was 15,628 miles.

Eighty-six percent of daily travel and $98 \%$ of all outings are less than 70 miles and are well within the advertised range of a BEV. Some daily travel exceeds this 70-mile range, but this is rather infrequent and random, suggesting that with a PHEV available to support this vehicle with the longer trips, the mission can be accomplished. Single, long-duration outings are not typical with this vehicle.

With $67 \%$ of daily travel and $92 \%$ of outings less than 40 miles, the advertised range of a PHEV on CD mode only, the PHEV could provide significant travel on battery power. The PHEV may provide the acceptable performance of this vehicle should the PHEV meet other mission requirements. This pickup's roadwork assignment suggests that cargo capability may be an important requirement. PHEVs with this capability do exist and could provide an alternative for this vehicle. 
Vehicle 38768

\begin{tabular}{|l|l|c|}
\hline & Make/Model/Year & Chevrolet/Impala/2010 \\
\cline { 2 - 3 } & EPA Class Size & Sedan-Large \\
\hline Mission & Pool \\
\hline VIN & 2G1WA5EK8A1138768 \\
\cline { 2 - 3 } & Parking Location & 38768 \\
\hline Fleet Vehicle ID (VIN5) & Gas/ETH \\
\hline & Fuel Type & $18 / 29 / 22 ~ 14 / 22 / 17$ \\
\hline & EPA Label/MPG (City/Hwy) & $440 / 370$ \\
\cline { 2 - 3 } & EPA GHG Emissions (Grams $\left.\mathrm{CO}_{2} / \mathrm{Mi}\right)$ & Logger 71 \\
\hline & Study Logger ID & $45 / 64$ \\
\hline
\end{tabular}

\begin{tabular}{|l|c|c|c|c|}
\hline \multicolumn{5}{|c|}{ Vehicle 38768 Travel Summary } \\
\hline & $\begin{array}{c}\text { Per Day } \\
\text { Average/Peak }\end{array}$ & $\begin{array}{c}\text { Per Outing } \\
\text { Average/Peak }\end{array}$ & $\begin{array}{c}\text { Per Trip } \\
\text { Average/Peak }\end{array}$ & Total \\
\hline Travel Distance (Miles) & $17.5 / 205.3$ & $15.8 / 490.5$ & $2.6 / 198.0$ & 789 \\
\hline Travel Time (Minutes) & $44.0 / 263.0$ & $39.2 / 833.0$ & $6.6 / 221.0$ & 1,958 \\
\hline Idle Time (Minutes) & $11.1 / \mathrm{NA}$ & $10.0 / \mathrm{NA}$ & $1.7 / \mathrm{NA}$ & 499 \\
\hline
\end{tabular}

\begin{tabular}{|c|c|c|c|c|}
\hline \multicolumn{2}{|c|}{ Total Stops } & \multicolumn{2}{c|}{ Stop Duration } \\
\hline $\begin{array}{c}\text { Distance From } \\
\text { Home Base (Miles) }\end{array}$ & Stops & Percentages & Stop Duration (Hours) & Stops \\
\hline Less than 10 & 137 & $63.7 \%$ & Less than 2 & 166 \\
\hline 10 to 20 & 76 & $35.3 \%$ & 2 to 4 & 17 \\
\hline 20 to 40 & 1 & $0.5 \%$ & 4 to 8 & 6 \\
\hline 40 to 60 & 1 & $0.5 \%$ & Greater than 8 & 26 \\
\hline
\end{tabular}

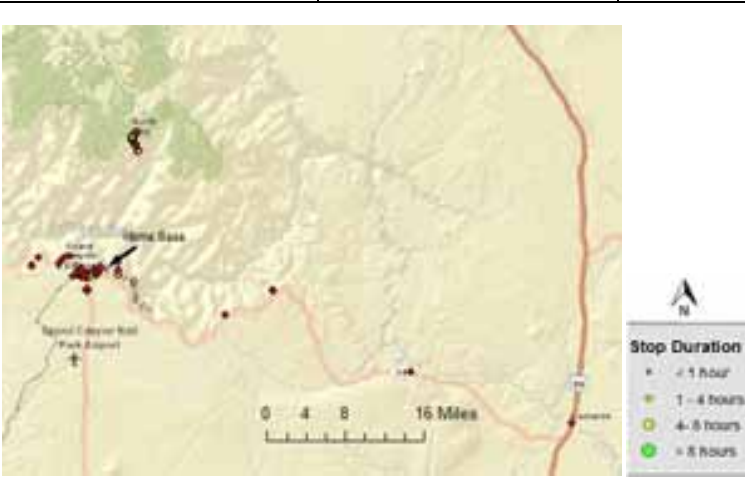

Figure D-10. Vehicle 38738 stops.

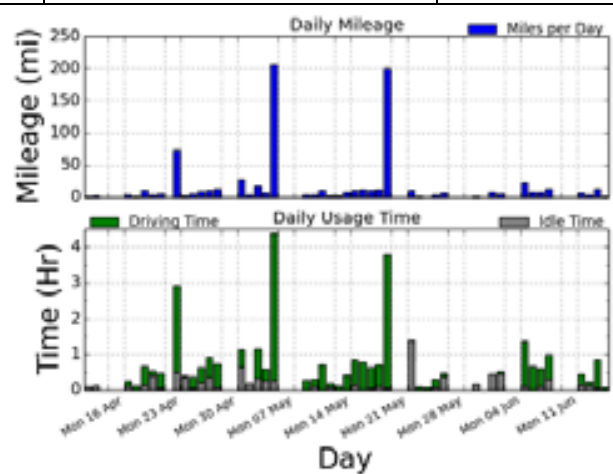

Figure D-11. Vehicle 38768 history. 

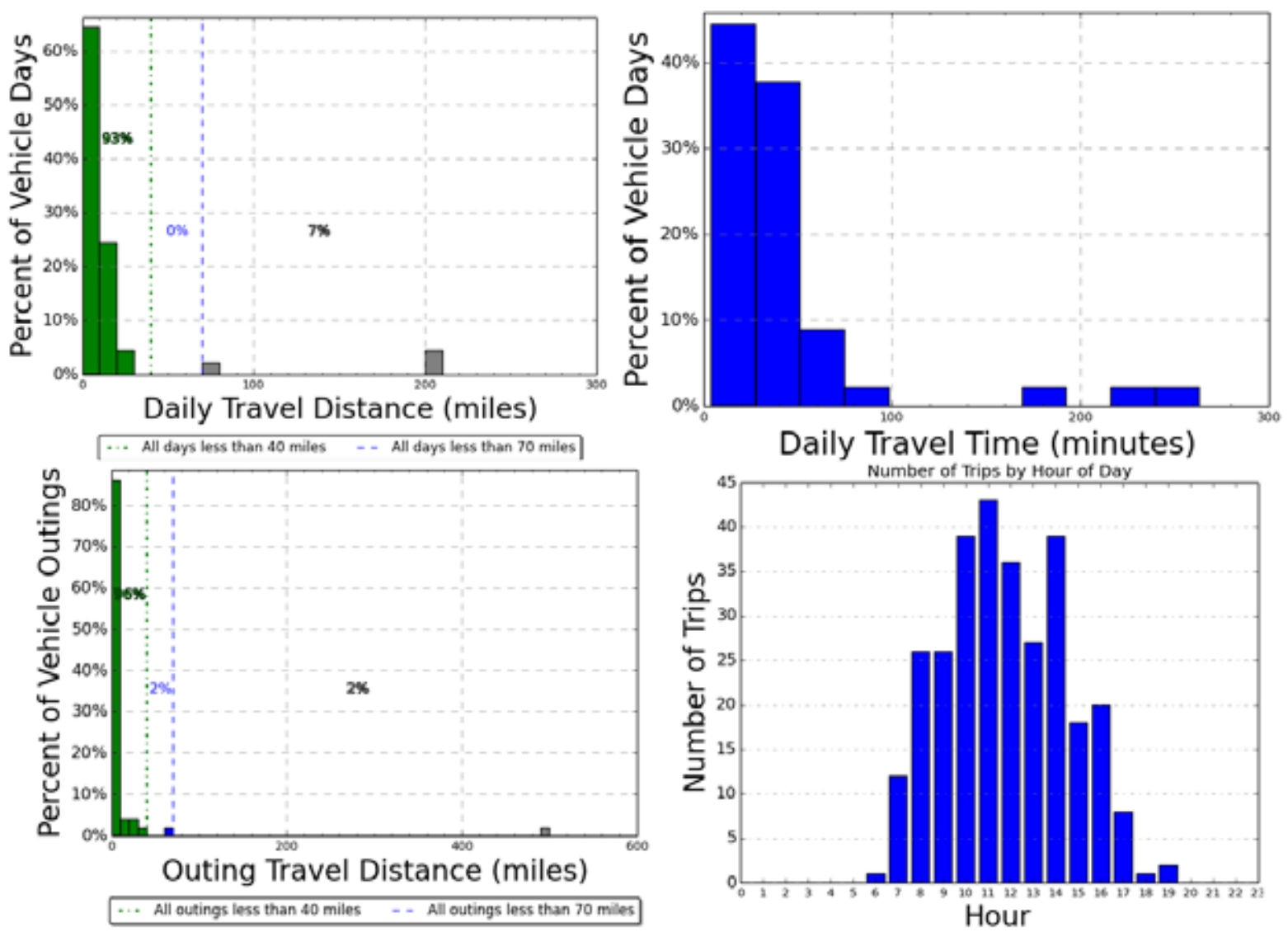

Figure D-7. Vehicle 38768 travel graphs.

\section{Vehicle 38768 Observations}

Logger 71 collected data on this vehicle for 45 days of the 64-day study period. Validation occurred on $98.7 \%$ of the vehicle data. Vehicle survey information on this vehicle indicates that it is used for administrative purposes supporting the Division of Concessions Management. A pool mission is implied.

The reported odometer reading was 16,871 miles, with an estimated usage of 7,000 miles/year.

Ninety-three percent of daily travel and $98 \%$ of outings are within the 70 -mile advertised range of a BEV. One outing of 490 miles occurred when the vehicle traveled to the North Rim and remained there for 2 weeks. Both days of daily travel at 200 miles were involved in this single outing. Even if charging stations were available at the North Rim, the extensive travel to transit between North and South Rims exceed the range of a BEV.

A PHEV may provide the acceptable performance of this vehicle. With $96 \%$ of outings and $93 \%$ of daily travel within the advertised 40-mile battery-only range, a PHEV provides an alternative for this vehicle. Several PHEV sedans are available. 


\begin{tabular}{|l|l|c|}
\hline & Make/Model/Year & Ford/Explorer/2010 \\
\hline EPA Class Size & SUV \\
\hline Mission & Pool \\
\hline VIN & 1 FMEU7DE4AUA67912 \\
\hline Parking Location & Pool \\
\hline Fleet Vehicle ID (VIN5) & 67912 \\
\hline Fuel Type & Gas \\
\hline EPA Label/MPG (City/Hwy/Combined) & $14 / 19 / 16$ \\
\hline & EPA GHG Emissions (Grams CO $\left.\mathrm{CO}_{2} / \mathrm{Mi}\right)$ & 555 \\
\hline & Study Logger ID & Logger 72 \\
\hline & Total Vehicle Days/Total Study Days & $25 / 60$ \\
\hline
\end{tabular}

\begin{tabular}{|l|c|c|c|c|}
\hline \multicolumn{5}{|c|}{ Vehicle 67912 Travel Summary } \\
\hline & $\begin{array}{c}\text { Per Day } \\
\text { Average/Peak }\end{array}$ & $\begin{array}{c}\text { Per Outing } \\
\text { Average/Peak }\end{array}$ & $\begin{array}{c}\text { Per Trip } \\
\text { Average/Peak }\end{array}$ & Total \\
\hline Travel Distance (Miles) & $38.7 / 284.0$ & $21.0 / 477.4$ & $7.2 / 147.7$ & 968 \\
\hline Travel Time (Minutes) & $64 / 358.0$ & $34.6 / 636.0$ & $11.9 / 164.0$ & 1,591 \\
\hline Idle Time (Minutes) & $5.8 / \mathrm{NA}$ & $3.2 / \mathrm{NA}$ & $1.1 / \mathrm{NA}$ & 146 \\
\hline
\end{tabular}

\begin{tabular}{|c|c|c|c|c|}
\hline \multicolumn{2}{|c|}{ Total Stops } & \multicolumn{2}{c|}{ Stop Duration } \\
\hline $\begin{array}{c}\text { Distance From } \\
\text { Home Base (Miles) }\end{array}$ & Stops & Percentages & Stop Duration (Hours) & Stops \\
\hline Less than 10 & 96 & 80.7 & Less than 2 & 84 \\
\hline 10 to 20 & 7 & $5.9 \%$ & 2 to 4 & 9 \\
\hline 20 to 40 & 0 & $0 \%$ & 4 to 8 & 5 \\
\hline 40 to 60 & 16 & $13.4 \%$ & Greater than 8 & 21 \\
\hline
\end{tabular}

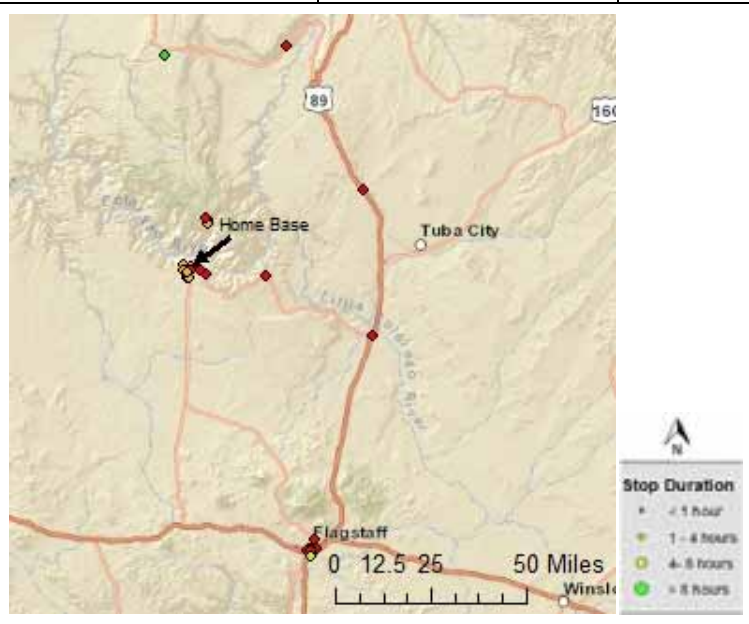

Figure D-16. Vehicle 67912 stops.

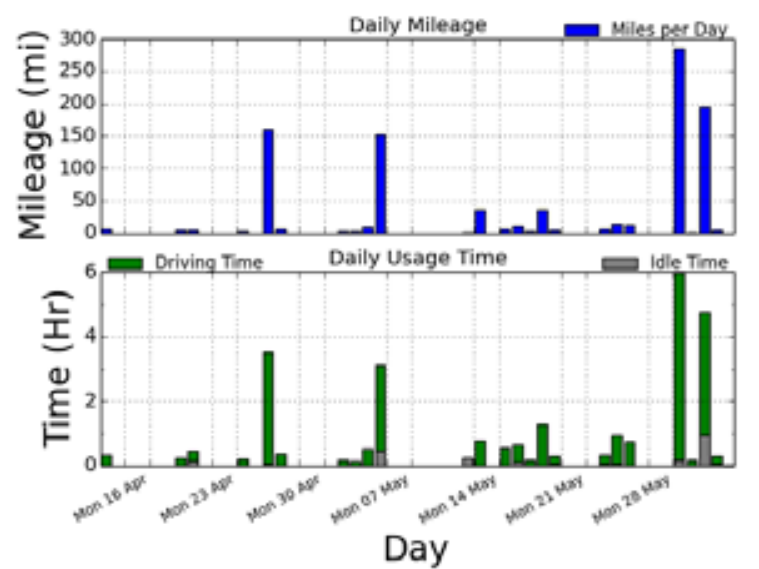

Figure D-17. Vehicle 67912 history. 

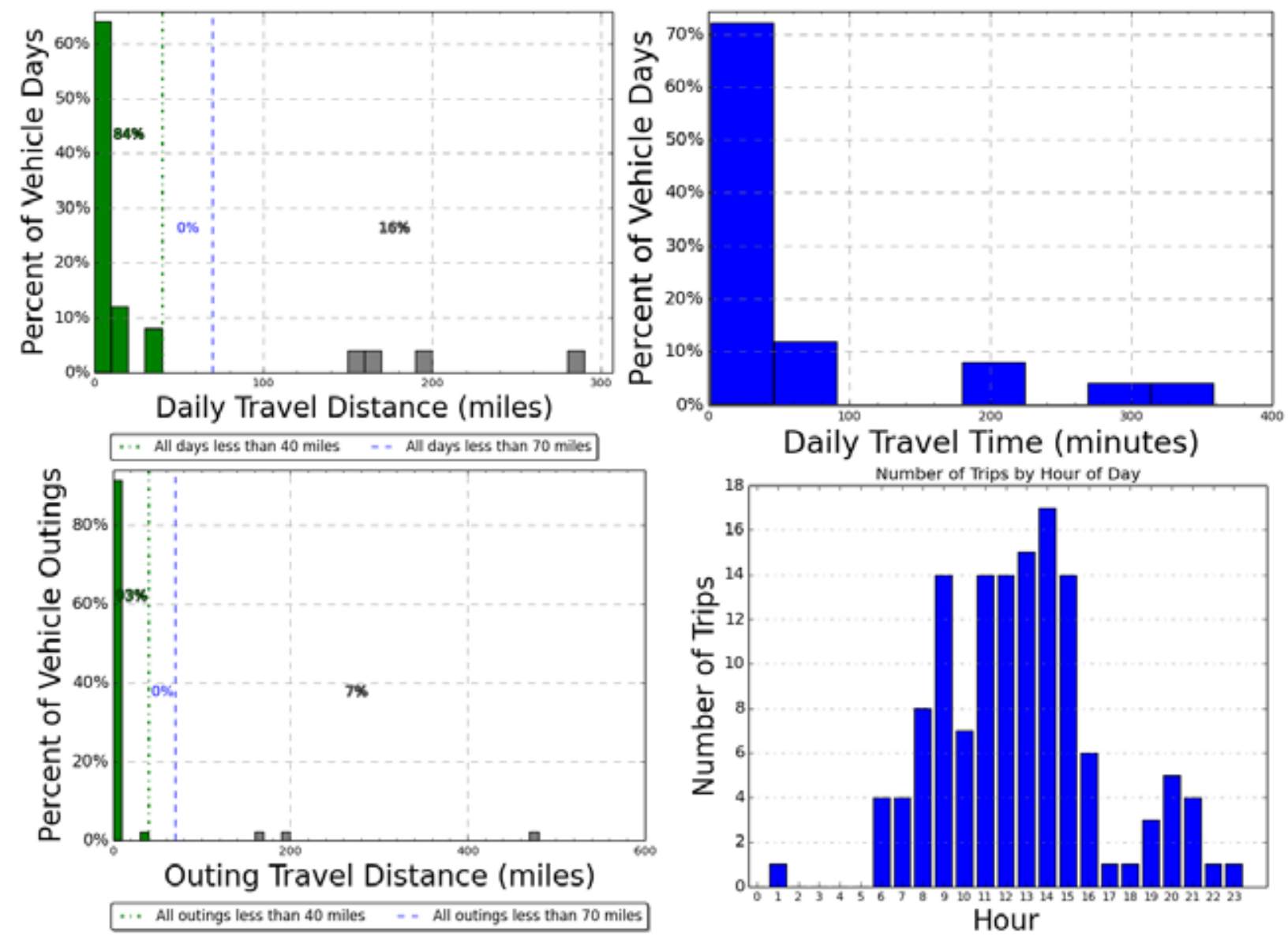

Figure D-8. Vehicle 67912 travel graphs.

\section{Vehicle 67912 Observations}

Logger 72 collected data on this vehicle for 25 days of the 60-day study. Validation occurred on $98.9 \%$ of the vehicle data. Survey information indicates this vehicle is used for administrative purposes and trips and is typically parked at the Division of Science and Resource Education. A pool mission is implied.

The reported odometer reading was 23,792 miles, with an estimated usage of 10,000 miles/year.

Long daily travel occurred on four occasions involving travel to Flagstaff and to Kaibab and the North Rim. Several days were spent at the North Rim; therefore, the maximum outing reported exceeds the maximum daily travel. While these excursions were not necessarily typical, they were frequent enough to suggest a BEV may not be suitable to replace this particular vehicle. However, as a pool vehicle, a BEV replacing this vehicle could be paired with a PHEV that could be used on the longer trips. (see Vehicle 73.) A charging station at the North Rim would support charging as well.

Eighty-four percent of daily travel and $93 \%$ of outings are within the 70 -mile advertised range of a BEV and the same percentages of daily travel and outings are within the 40-mile advertised battery only range of a PHEV. 


\begin{tabular}{|c|c|c|}
\hline in & Make/Model/Year & Dodge/Durango/2008 \\
\hline$=$ & EPA Class Size & SUV \\
\hline$f_{2}=\frac{1}{m} x^{2}$ & Mission & Pool \\
\hline & VIN & 1D8HB38N48F145115 \\
\hline & Parking Location & Albright Ave, GC Village \\
\hline & Fleet Vehicle ID (VIN5) & 45115 \\
\hline & Fuel Type & Gas/ETH \\
\hline & EPA Label/MPG (City/Hwy) & $13 / 17 / 15 \quad 9 / 12 / 10$ \\
\hline & EPA GHG Emissions (Grams $\mathrm{CO}_{2} / \mathrm{Mi}$ ) & $529 / 630$ \\
\hline & Study Logger ID & Logger 73 \\
\hline & Total Vehicle Days/Total Study Days & $48 / 60$ \\
\hline
\end{tabular}

\begin{tabular}{|l|c|c|c|c|}
\hline \multicolumn{5}{|c|}{ Vehicle 227 Travel Summary } \\
\hline & $\begin{array}{c}\text { Per Day } \\
\text { Average/Peak }\end{array}$ & $\begin{array}{c}\text { Per Outing } \\
\text { Average/Peak }\end{array}$ & $\begin{array}{c}\text { Per Trip } \\
\text { Average/Peak }\end{array}$ & Total \\
\hline Travel Distance (Miles) & $39.7 / 360.4$ & $42.3 / 369.6$ & $6.1 / 142.1$ & 1,905 \\
\hline Travel Time (Minutes) & $73.0 / 378.0$ & $77.8 / 399.0$ & $11.2 / 144.0$ & 3,500 \\
\hline Idle Time (Minutes) & $19.5 / \mathrm{NA}$ & $20.8 / \mathrm{NA}$ & $3.0 / \mathrm{NA}$ & 938 \\
\hline
\end{tabular}

\begin{tabular}{|c|c|c|c|c|}
\hline \multicolumn{2}{|c|}{ Total Stops } & \multicolumn{2}{c|}{ Stop Duration } \\
\hline $\begin{array}{c}\text { Distance From } \\
\text { Home Base (Miles) }\end{array}$ & Stops & Percentages & Stop Duration (Hours) & Stops \\
\hline Less than 10 & 101 & $55.2 \%$ & Less than 2 & 141 \\
\hline 10 to 20 & 77 & $42.1 \%$ & 2 to 4 & 12 \\
\hline 20 to 40 & 1 & $0.5 \%$ & 4 to 8 & 7 \\
\hline 40 to 60 & 4 & $2.2 \%$ & Greater than 8 & 23 \\
\hline
\end{tabular}

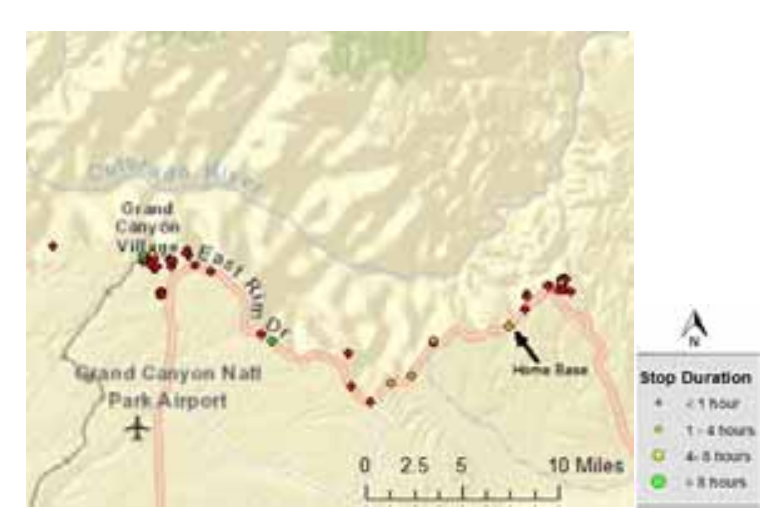

Figure D-19. Vehicle 227 stops.

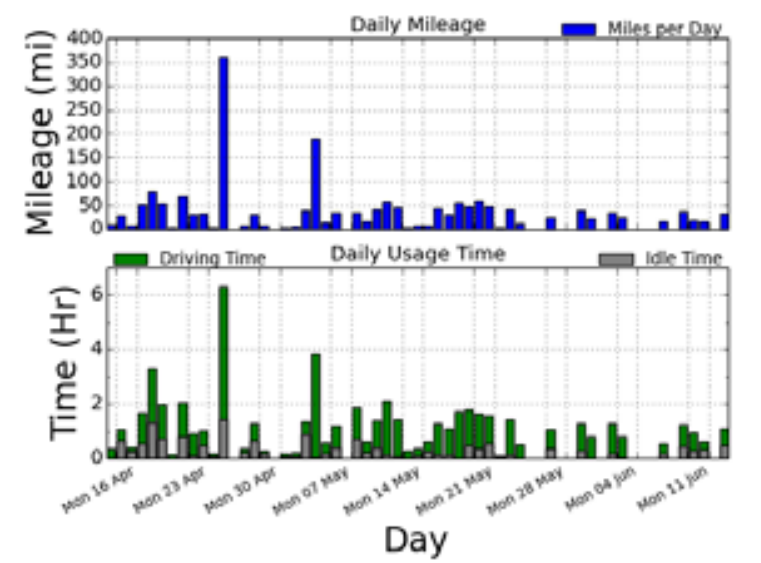

Figure D-20. Vehicle 227 history. 

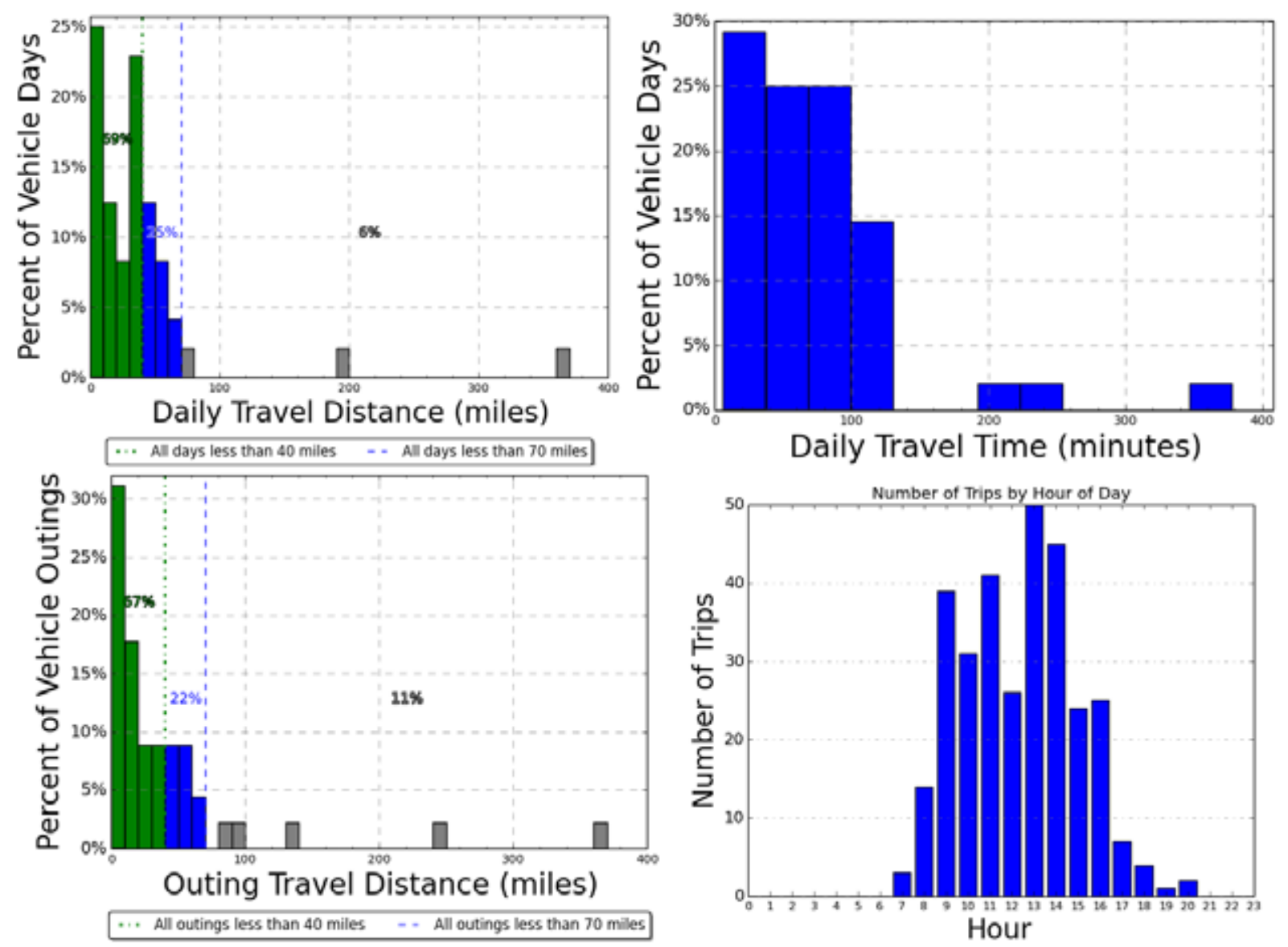

Daily Travel Time (minutes)

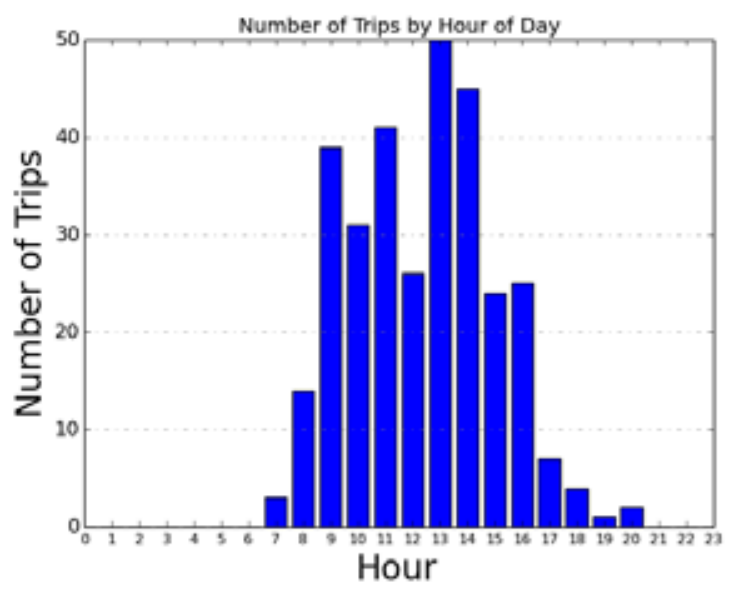

Figure D-9. Vehicle 227 travel graphs.

\section{Vehicle 227 Observations}

Logger 73 collected data on this vehicle for 48 days of the 60 -day study period. Validation occurred on $94.9 \%$ of the vehicle data. Vehicle survey information indicates the Division of Interpretation and Resource Education uses this vehicle for trips to the South Rim from Desert View. A pool mission is implied.

The reported odometer reading was 54,984 miles, with an estimated usage of 13,750 miles/year.

The longest daily outing of 360 miles occurred on April 24 on a multi-day excursion to Kaibab, Coconino, and Williams, Arizona. The multi-day excursion is why the maximum outing distance is greater than the daily maximum. Ninety-four percent of daily travel and $89 \%$ of outings are within the typically advertised 70 -mile range of a BEV, while $69 \%$ of daily travel and $67 \%$ of outings were within the typically advertised 40-mile battery-only range of a PHEV. The longer daily travel would not support the direct replacement of this vehicle with a BEV, but as a pool vehicle, a replacement PHEV here could also assume the longer trips of Vehicle 72. 


\begin{tabular}{|l|l|c|}
\hline & Make/Model/Year & Ford/Ranger/2007 \\
\hline EPA Class Size & Pickup Trucks 4WD \\
\hline Mission & Support \\
\hline VIN & $\begin{array}{c}\text { South Entrance Road, Grand } \\
\text { Canyon Village }\end{array}$ \\
\cline { 2 - 3 } & Parking Location & 90661 \\
\hline Fleet Vehicle ID (VIN5) & Gas \\
\hline Fuel Type & $21 / 26 / 23$ \\
\hline EPA Label/MPG (City/Hwy) & 386 \\
\hline & EPA GHG Emissions (Grams CO $\left.\mathrm{CO}_{2} / \mathrm{Mi}\right)$ & Logger 74 \\
\hline & Study Logger ID & $42 / 57$ \\
\hline
\end{tabular}

\begin{tabular}{|l|c|c|c|c|}
\hline \multicolumn{5}{|c|}{ Vehicle 90661 Travel Summary } \\
\hline & $\begin{array}{c}\text { Per Day } \\
\text { Average/Peak }\end{array}$ & $\begin{array}{c}\text { Per Outing } \\
\text { Average/Peak }\end{array}$ & $\begin{array}{c}\text { Per Trip } \\
\text { Average/Peak }\end{array}$ & Total \\
\hline Travel Distance (Miles) & $90.1 /$ & $122.1 /$ & $11.9 /$ & 3,785 \\
\hline Travel Time (Minutes) & $177 /$ & $240.4 /$ & $23.4 /$ & 7,451 \\
\hline Idle Time (Minutes) & $61.5 / \mathrm{NA}$ & $83.3 / \mathrm{NA}$ & $8.1 / \mathrm{NA}$ & 2,583 \\
\hline
\end{tabular}

\begin{tabular}{|c|c|c|c|c|}
\hline \multicolumn{2}{|c|}{ Total Stops } & \multicolumn{2}{c|}{ Stop Duration } \\
\hline $\begin{array}{c}\text { Distance From } \\
\text { Home Base (Miles) }\end{array}$ & Stops & Percentages & Stop Duration (Hours) & Stops \\
\hline Less than 10 & 50 & $24.2 \%$ & Less than 2 & 145 \\
\hline 10 to 20 & 23 & $11.1 \%$ & 2 to 4 & 11 \\
\hline 20 to 40 & 0 & $0 \%$ & 4 to 8 & 11 \\
\hline 40 to 60 & 134 & $64.8 \%$ & Greater than 8 & 40 \\
\hline
\end{tabular}

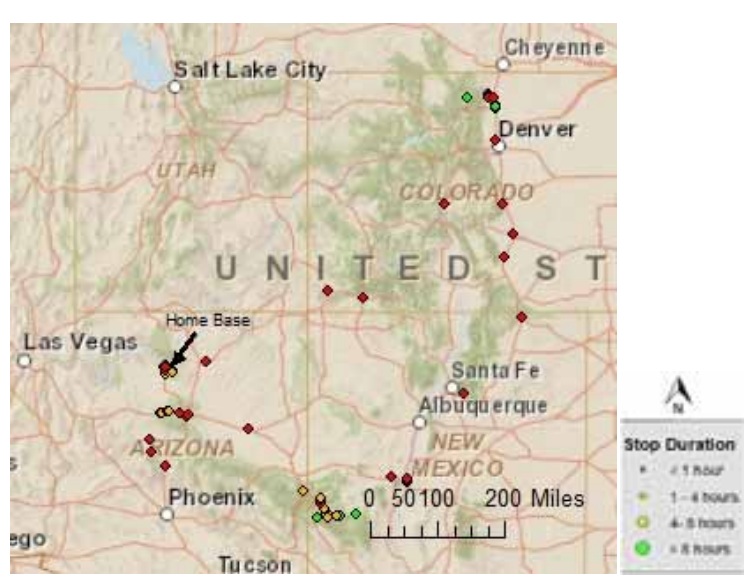

Figure D-22. Vehicle 90661 stops.

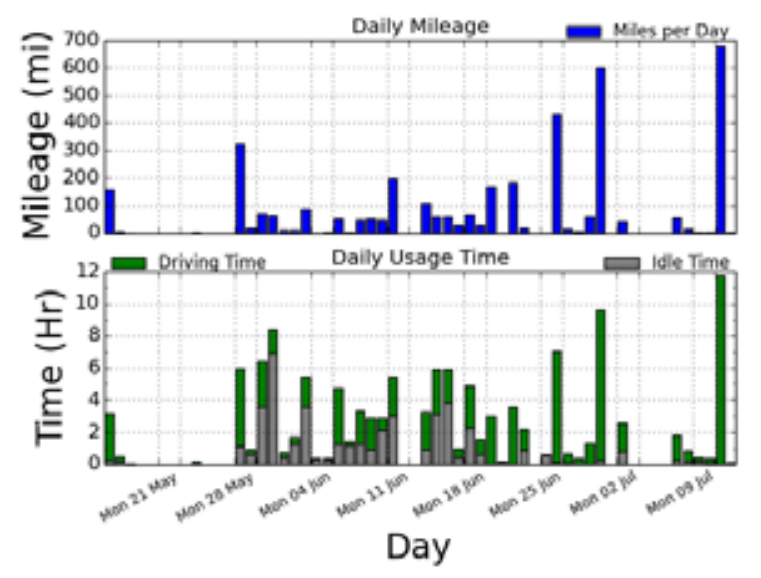

Figure D-23. Vehicle 90661 history. 

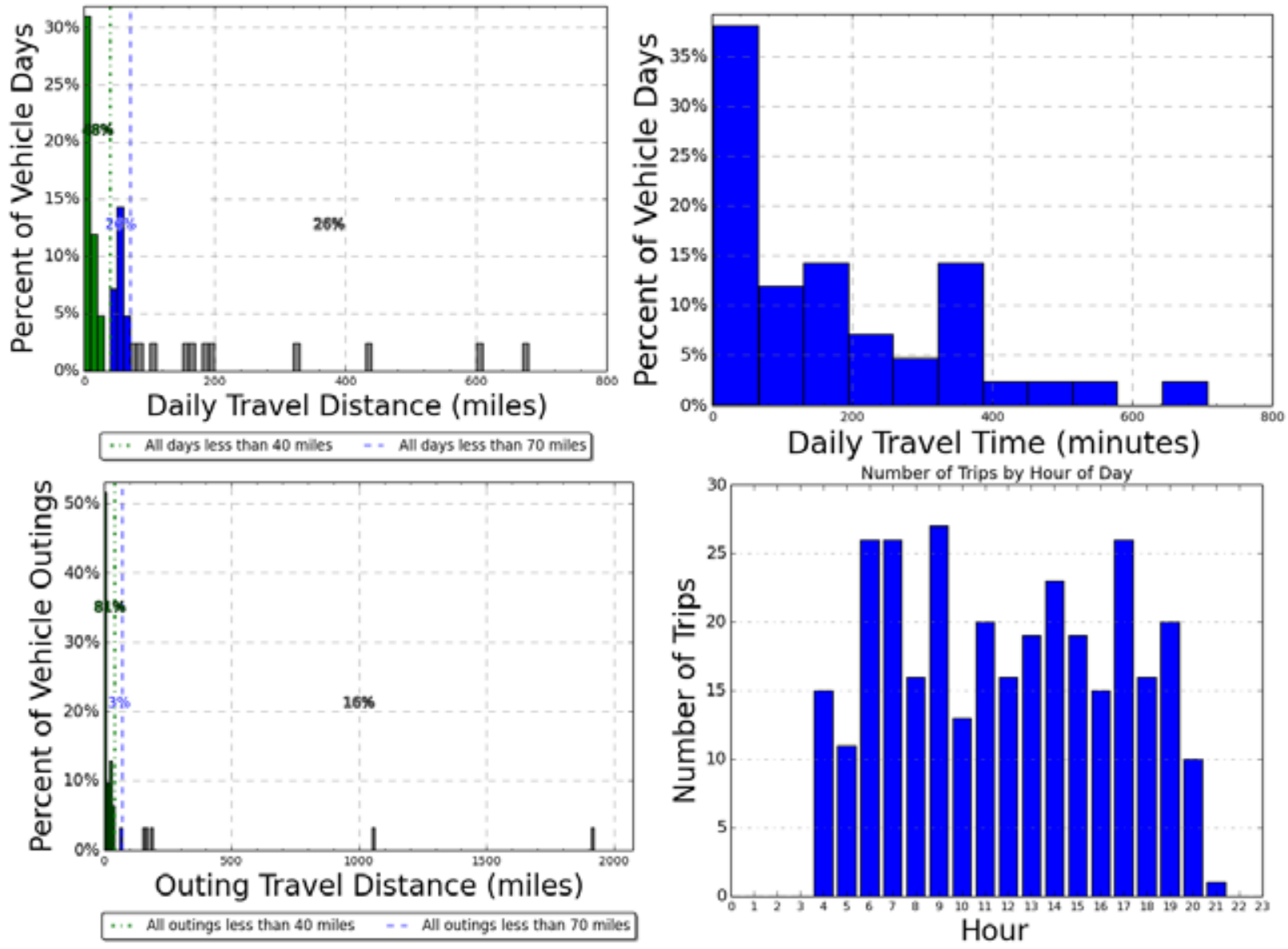

Figure D-10. Vehicle 90661 travel graphs.

\section{Vehicle 90661 Observations}

Logger 74 collected data on this vehicle for 42 days of the 57-day study period. Validation occurred on $92.7 \%$ of the vehicle data. The vehicle survey reports that this vehicle is used for fire response and a support mission is implied.

The reported odometer reading was 27,321 miles, with an estimated usage of 5,464 miles/year.

This vehicle traveled long distances on several occasions to Flagstaff and other locations in Arizona, as well as spending several days in New Mexico. Even so, $74 \%$ of travel days and $84 \%$ of outings were within the 70 -mile advertised range of a BEV and $48 \%$ of travel days and $81 \%$ of outings are within the 40-mile advertised battery-only range of a PHEV.

A PHEV may provide the acceptable performance of this vehicle should the PHEV meet other mission requirements. Information is unavailable suggesting special requirements exist other than this vehicle is a pickup for which replacement PEVs are available. 


\begin{tabular}{|c|c|c|}
\hline$\Rightarrow$ & Make/Model/Year & Chevrolet/HHR/2010 \\
\hline 1 & EPA Class Size* & SUV \\
\hline 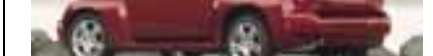 & Mission & Pool \\
\hline & VIN & 3GNBAADBXAS520389 \\
\hline & Parking Location & $\begin{array}{c}\text { South Entrance Road, Grand } \\
\text { Canyon Village }\end{array}$ \\
\hline & Fleet Vehicle ID (VIN5) & 20389 \\
\hline & Fuel Type & $\mathrm{Gas} / \mathrm{ETH}$ \\
\hline & EPA Label/MPG (City/Hwy)* & $22 / 32 / 26 \quad 16 / 23 / 19$ \\
\hline & EPA GHG Emissions (Grams $\left.\mathrm{CO}_{2} / \mathrm{Mi}\right)^{*}$ & $342 / 331$ \\
\hline & Study Logger ID & Logger 75 \\
\hline & Total Vehicle Days/Total Study Days & $35 / 77$ \\
\hline
\end{tabular}

\begin{tabular}{|l|c|c|c|c|}
\hline \multicolumn{5}{|c|}{ Vehicle 20389 Travel Summary } \\
& $\begin{array}{c}\text { Per Day } \\
\text { Average/Peak }\end{array}$ & $\begin{array}{c}\text { Per Outing } \\
\text { Average/Peak }\end{array}$ & $\begin{array}{c}\text { Per Trip } \\
\text { Average/Peak }\end{array}$ & Total \\
\hline Travel Distance (Miles) & $39.3 / 283.7$ & $27.5 / 495.2$ & $7.2 / 133.1$ & 1,377 \\
\hline Travel Time (Minutes) & $74 / 354$ & $51.9 / 657$ & $13.7 / 152.0$ & 2,595 \\
\hline Idle Time (Minutes) & $19.1 / \mathrm{NA}$ & $13.4 / \mathrm{NA}$ & $3.5 / \mathrm{NA}$ & 670 \\
\hline
\end{tabular}

\begin{tabular}{|c|c|c|c|c|}
\hline \multicolumn{2}{|c|}{ Total Stops } & \multicolumn{2}{c|}{ Stop Duration } \\
\hline $\begin{array}{c}\text { Distance From } \\
\text { Home Base (Miles) }\end{array}$ & Stops & Percentages & Stop Duration (Hours) & Stops \\
\hline Less than 10 & 88 & $74.6 \%$ & Less than 2 & 90 \\
\hline 10 to 20 & 7 & $5.9 \%$ & 2 to 4 & 4 \\
\hline 20 to 40 & 0 & $0 \%$ & 4 to 8 & 3 \\
\hline 40 to 60 & 23 & $19.4 \%$ & Greater than 8 & 21 \\
\hline
\end{tabular}

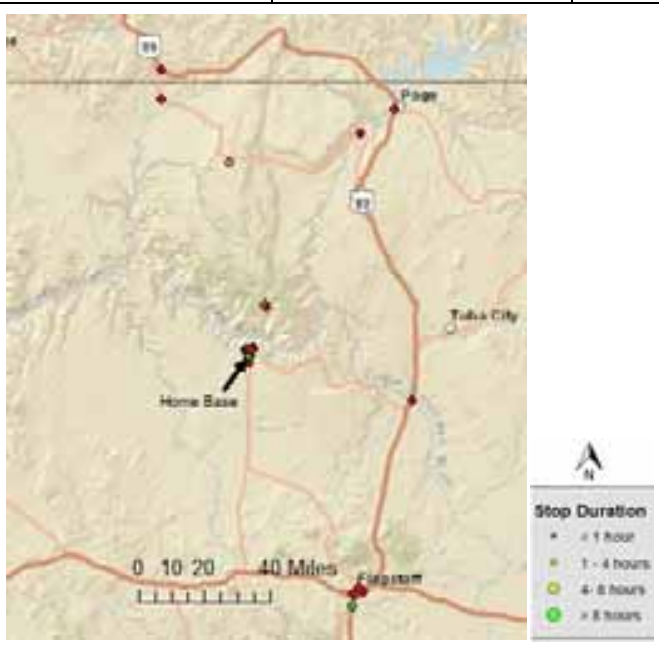

Figure D-25. Vehicle 20389 stops.

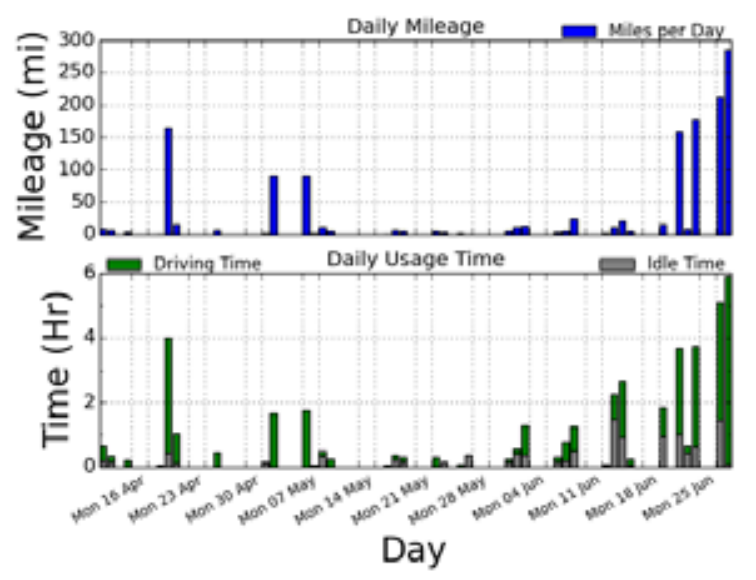

Figure D-26. Vehicle 20389 history. 

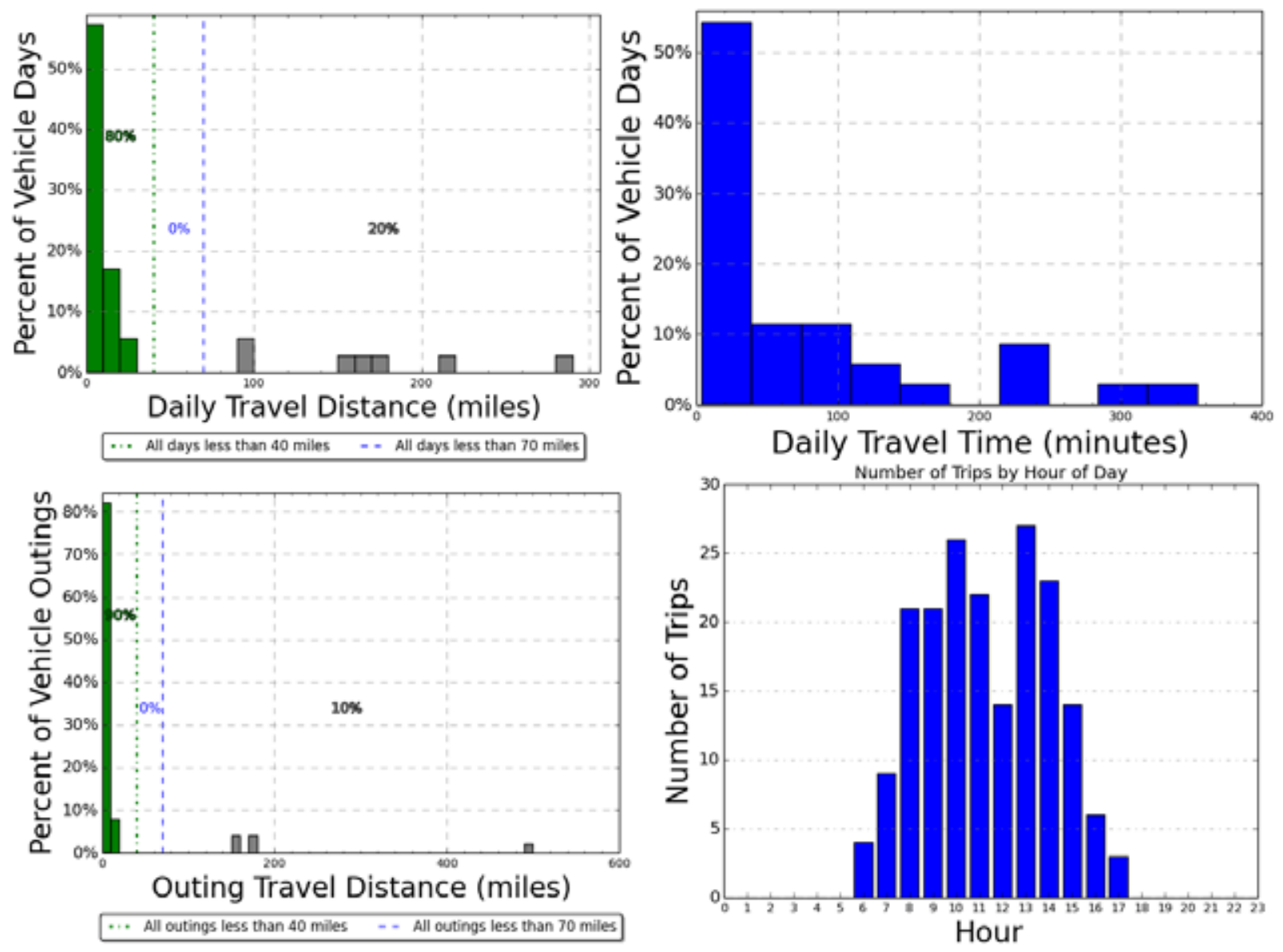

Figure D-11. Vehicle 20389 travel graphs.

\section{Vehicle 20389 Observations}

Logger 75 collected data on this vehicle for 35 days of the 77-day study period. Validation occurred on $94.3 \%$ of the vehicle data. Vehicle survey information indicates the Division of Administration uses this vehicle for trips within the park, to Flagstaff, and other travel. A pool mission is implied.

The reported odometer reading was 9,987 miles, with an estimated usage of 4,994 miles/year.

This vehicle travels out of the park on several occasions to Flagstaff and to the North Rim. It remained overnight at the North Rim so that the maximum outing recorded exceeds the maximum daily travel.

Eighty percent of daily travel and $90 \%$ of outings are within both the 70 -mile advertised range of a BEV and within the 40-mile battery-only range of a PHEV. The balance of the travel is outside these ranges; therefore, a BEV is not a recommended replacement for this specific vehicle. While charging stations at the North Rim would be beneficial, it would not be sufficient to replace this vehicle with a BEV.

However, this vehicle is a pool vehicle and other vehicles in the pool, if replaced by a BEV, could support the shorter distances, while a PHEV replacing this vehicle could support the longer distances. 
Vehicle 17523

\begin{tabular}{|l|l|c|}
\hline & Make/Model/Year & Ford/Focus/2012 \\
\hline EPA Class Size* & Sedan-Compact \\
\hline Mission & Support \\
\hline VIN & 1FAHP3F26CL217523 \\
\hline Parking Location & Albright Ave, GC Village \\
\hline Fleet Vehicle ID (VIN5) & Gas/ETH \\
\hline Fuel Type & $28 / 38 / 31$ 19/28/22 \\
\hline EPA Label/MPG (City/Hwy) & $287 / 286$ \\
\hline EPA GHG Emissions (Grams $\left.\mathrm{CO}_{2} / \mathrm{Mi}\right)$ & Logger 79 \\
\hline & Study Logger ID & $32 / 89$ \\
\hline
\end{tabular}

\begin{tabular}{|l|c|c|c|c|}
\hline \multicolumn{5}{|c|}{ Vehicle 17523 Travel Summary } \\
\hline & $\begin{array}{c}\text { Per Day } \\
\text { Average/Peak }\end{array}$ & $\begin{array}{c}\text { Per Outing } \\
\text { Average/Peak }\end{array}$ & $\begin{array}{c}\text { Per Trip } \\
\text { Average/10.0/Peak }\end{array}$ & Total \\
\hline Travel Distance (Miles) & $52.9 / 353.4$ & $54.6 / 882.2$ & $13.9 / 190.1$ & 1,693 \\
\hline Travel Time (Minutes) & $68 / 342.0$ & $70.2 / 883.0$ & $17.8 / 160.0$ & 2,176 \\
\hline Idle Time (Minutes) & $2.3 / \mathrm{NA}$ & $2.3 / \mathrm{NA}$ & $0.6 / \mathrm{NA}$ & 72 \\
\hline
\end{tabular}

\begin{tabular}{|c|c|c|c|c|}
\hline \multicolumn{2}{|c|}{ Total Stops } & \multicolumn{2}{c|}{ Stop Duration } \\
\hline $\begin{array}{c}\text { Distance From } \\
\text { Home Base (Miles) }\end{array}$ & Stops & Percentages & Stop Duration (Hours) & Stops \\
\hline Less than 10 & 69 & $59 \%$ & Less than 2 & 64 \\
\hline 10 to 20 & 0 & $0 \%$ & 2 to 4 & 13 \\
\hline 20 to 40 & 0 & $0 \%$ & 4 to 8 & 8 \\
\hline 40 to 60 & 48 & $41 \%$ & Greater than 8 & 32 \\
\hline
\end{tabular}

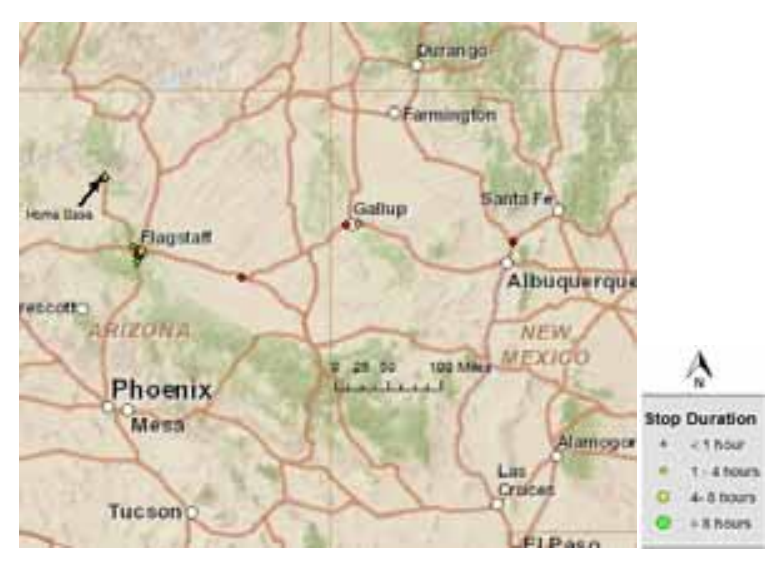

Figure D-28. Vehicle 17523 stops.

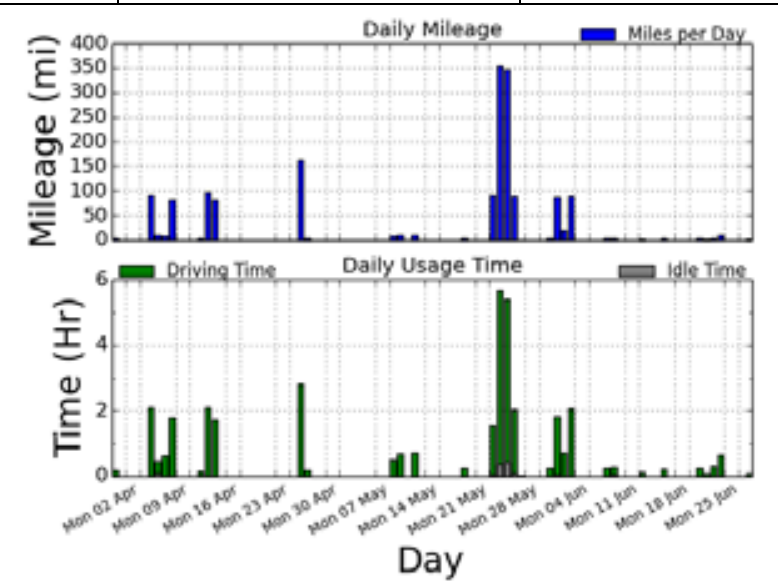

Figure D-29. Vehicle 17523 history. 

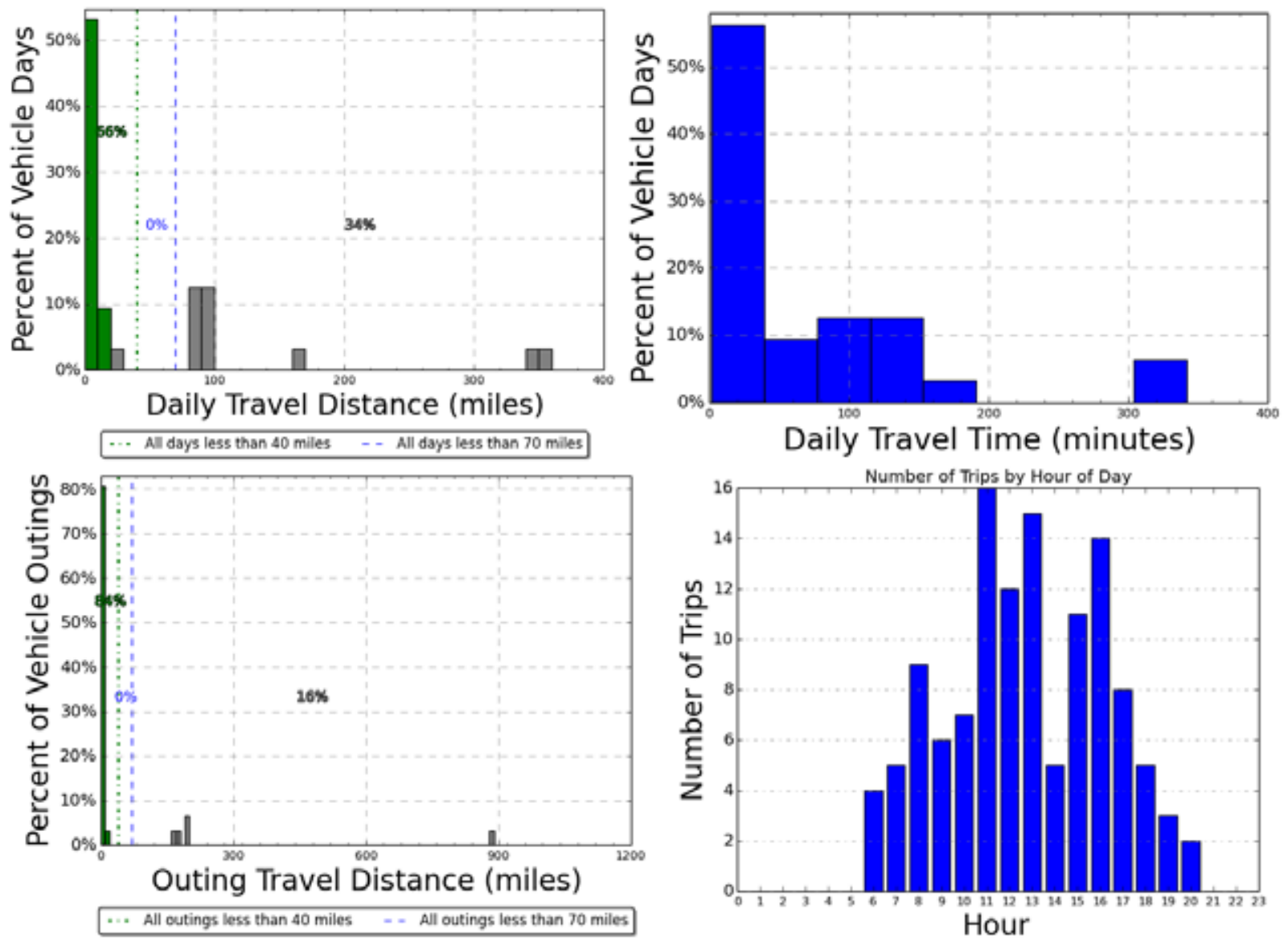

Figure D-12. Vehicle 17523 travel graphs.

\section{Vehicle 230 Observations}

Logger 79 collected data on this vehicle for 32 days of the 89-day study period. Validation occurred on $98.1 \%$ of the vehicle data. Vehicle survey information indicates this vehicle is assigned to Resource Management and is used by the chief and deputy for trips within the park, to Flagstaff, and other locations. A support mission is implied.

The reported odometer reading was 3,621 miles, with an estimated usage of 7,000 miles/year.

This vehicle's travel is characterized by mostly local travel, with longer excursions at infrequent times. The longest travel days occurred on May 22 through 24 on an excursion to Bernalillo, New Mexico. This several day travel is why the maximum outing recorded exceeds the maximum daily travel figures.

Fifty-six percent of daily travel and $84 \%$ of outings are within the 70 -mile advertised range of a BEV and within the 40-mile advertised battery-only advertised range of a PHEV.

The longer travel days suggest a BEV would not be a suitable replacement for this vehicle; however, PHEVs are available in the sedan body type. A PHEV would provide the opportunity for savings during local travel times. 
Vehicle Logger 69685

\begin{tabular}{|l|l|c|}
\hline Make/Model/Year & Ford/Escape/2011 \\
\hline EPA Class Size & SUV \\
\hline Mission & Support \\
\hline VIN & $\begin{array}{c}\text { South Entrance Road, Grand } \\
\text { Canyon Village }\end{array}$ \\
\hline Parking Location & 69685 \\
\hline Fleet Vehicle ID (VIN5) & Gas/ETH \\
\hline Fuel Type & $18 / 23 / 2013 / 17 / 14$ \\
\hline EPA Label / MPG (City/Hwy) & $444 / 450$ \\
\hline & EPA GHG Emissions (Grams CO $\left.\mathrm{CO}_{2} / \mathrm{Mi}\right)$ & Logger 80 \\
\hline & Study Logger ID & $28 / 59$ \\
\hline
\end{tabular}

\begin{tabular}{|l|c|c|c|c|}
\hline \multicolumn{5}{|c|}{ Vehicle 6968Travel Summary } \\
\hline & $\begin{array}{c}\text { Per Day } \\
\text { Average/Peak }\end{array}$ & $\begin{array}{c}\text { Per Outing } \\
\text { Average/Peak }\end{array}$ & $\begin{array}{c}\text { Per Trip } \\
\text { Average/Peak }\end{array}$ & Total \\
\hline Travel Distance (Miles) & $88.3 / 368.6$ & $68.7 / 365.8$ & $15.8 / 202.1$ & 2,472 \\
\hline Travel Time (Minutes) & $110 / 422.0$ & $85.6 / 414.0$ & $19.6 / 203$ & 3,080 \\
\hline Idle Time (Minutes) & $15.8 / \mathrm{NA}$ & $12.3 / \mathrm{NA}$ & $2.8 / \mathrm{NA}$ & 441 \\
\hline
\end{tabular}

\begin{tabular}{|c|c|c|c|c|}
\hline \multicolumn{2}{|c|}{ Total Stops } & \multicolumn{2}{c|}{ Stop Duration } \\
\hline $\begin{array}{c}\text { Distance From } \\
\text { Home Base (Miles) }\end{array}$ & Stops & Percentages & Stop Duration (Hours) & Stops \\
\hline Less than 10 & 61 & $58.1 \%$ & Less than 2 & 79 \\
\hline 10 to 20 & 5 & $4.8 \%$ & 2 to 4 & 7 \\
\hline 20 to 40 & 0 & $0 \%$ & 4 to 8 & 3 \\
\hline 40 to 60 & 39 & $37.1 \%$ & Greater than 8 & 16 \\
\hline
\end{tabular}

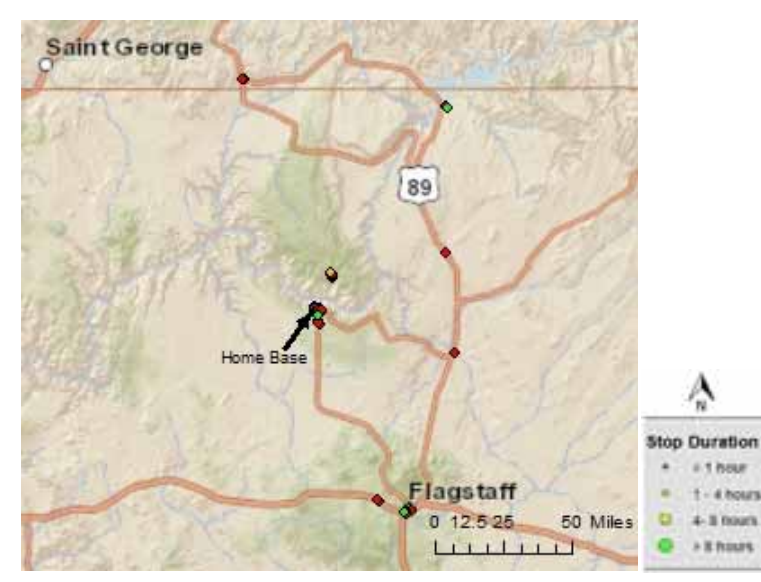

Figure D-31. Vehicle 69685 stops.

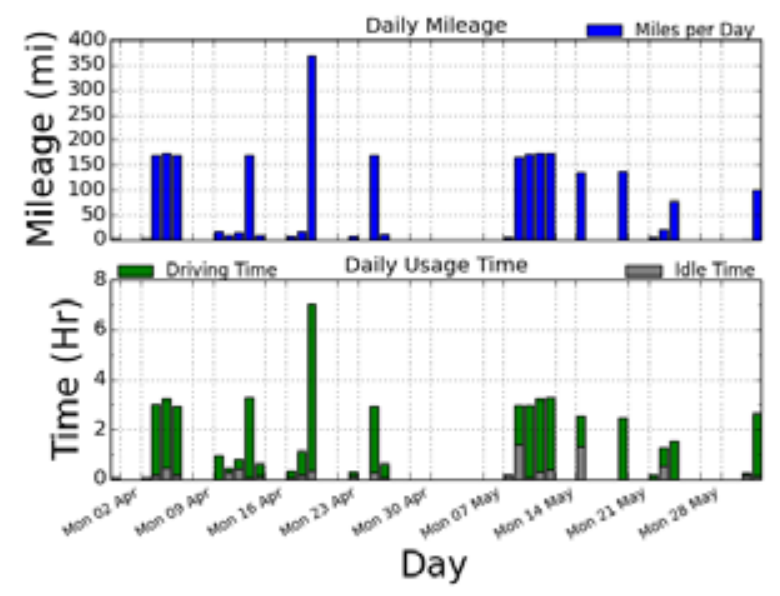

Figure D-32. Vehicle 69685 history. 

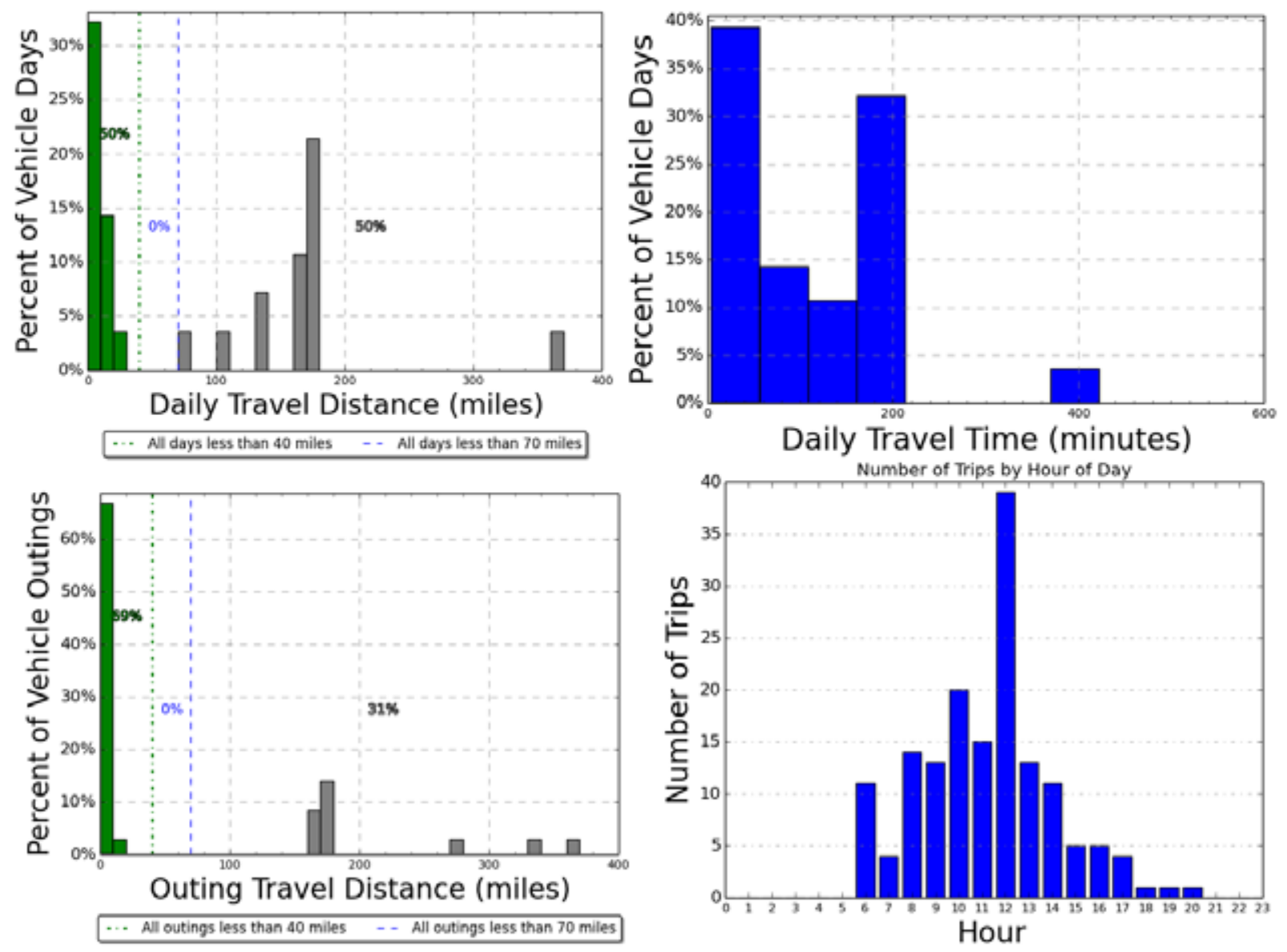

Figure D-13. Vehicle 69685travel graphs.

\section{Vehicle 69685 Observations}

Logger 80 collected data on this vehicle for 28 days of the 59-day study period. Validation occurred on $96.9 \%$ of the vehicle data. The project survey indicates the Engineering in Division of Facility Management uses this vehicle for professional services, environmental monitoring, and travel. A support mission is implied.

The reported odometer reading was 6,867 miles, with an estimated usage of 7,000 miles/year.

This vehicle's travel is characterized by frequent outings outside the South Rim area to Flagstaff and to Kanab, Utah, and to the North Rim. Half the daily travel exceeds the 70-mile advertised range of a BEV, suggesting a PHEV would be a more suitable PEV replacement. PHEV are available in the SUV vehicle body type. 